\title{
Quality aspects of distributed scatterers in polarimetric differential SAR interferometry
}

Adugna Girma Mullissa 


\section{Graduation committee}

Chair and Secretary

Prof.dr.ir. A. Veldkamp

Supervisor

Prof.dr.ir. A. Stein

University of Twente

University of Twente

Co-supervisor

Dr. V.A. Tolpekin

University of Twente

Members

Prof.dr.ir. M.G. Vosselman

University of Twente

University of Twente

Delft University of Technology

Prof.dr.ir. R.F. Hanssen

Prof.dr. P.M. Atkinson

Lancaster University

Prof.dr.ir. R.N.J. Veldhuis

University of Twente

ITC dissertation number 313

ITC, P.O. Box 217, 7500 AE Enschede, The Netherlands

ISBN: $\quad 978-90-365-4457-3$

DOI: $\quad 10.3990 / 1.9789036544573$

Printed by: ITC Printing Department, Enschede, The Netherlands

(C) Adugna Girma Mullissa, Enschede, The Netherlands

All rights reserved. No part of this publication may be reproduced without the prior written permission of the author.

-1 C Faculty of geo-information science and Earth observation 


\title{
QUALITY ASPECTS OF DISTRIBUTED SCATTERERS IN POLARIMETRIC DIFFERENTIAL SAR INTERFEROMETRY
}

\section{DISSERTATION}

\author{
to obtain \\ on the authority of the rector magnificus, \\ prof.dr. T.T.M. Palstra, \\ to be publicly defended \\ on Friday, December $22^{\text {nd }}, 2017$ at 16.45 hrs \\ by \\ Adugna Girma Mullissa \\ born on May $1^{\text {st }}, 1980$ \\ in Addis Abeba, Ethiopia
}

the degree of doctor at the University of Twente,

on account of the decision of the graduation committee, 
This dissertation is approved by:

Prof.dr.ir. A. Stein (supervisor)

Dr. V.A. Tolpekin (co-supervisor) 
To my family 



\section{Summary}

Ground deformation measurements deliver valuable information for the understanding of natural hazards such as landslides, ground subsidence, earthquakes and volcanism. Satellite based SAR interferometry provides detailed, cost effective measurements for this purpose. The quality of these measurements, however, is often degraded by decorrelation. Here quality is defined as the number of measurement points and accuracy of deformation measurements from these points. The degree of decorrelation is in particular severe in natural environments where the number of coherent targets is limited. Hence, improving the quality of deformation measurements in natural environments is important to improve their usability.

This research exploits the polarimetric diversity provided by fully and partially polarized SAR images to increase the number of measurement points and deformation measurement accuracy. It is composed of three major topics. First, the performance of three polarimetric optimization methods has been analyzed and evaluated to select the polarization state that is least affected by decorrelation. This was done in a single and double phase center scenario. These scenarios are applied on dual and quad polarized SAR images acquired from a completely natural environment in Ethiopia. The purpose was to quantify the coherence improvement in different types of distributed scatterers. Bias in the coherence estimation of the different optimizers was investigated from simulated PolInSAR image. Deformations observed from three different polarimetric optimization methods were compared with the deformation values obtained from an in-situ GPS. The applied polarimetric optimization routines substantially improved coherence estimation. These routines have a lower estimation bias as compared to the traditionally used single polarization channel.

Second, we developed a spatial filter using statistical homogeneous pixels (SHP) based upon the scattering mechanism to improve the signal to noise ratio of interferometric phase over distributed scatterers. This method was first developed to estimate polarimetric coherency matrix for a single image. It improved the estimation of coherency matrix by avoiding the indiscriminate use of moving averages. Improvement was achieved by iteratively classifying and refining the estimate of the coherency matrix. It derived the scattering mechanism by applying the Cloude-Pottier eigenvalue-eigenvector decomposi- 
tion technique whereas the Wishart distance measure was used to classify the scattering mechanism. Classification of scattering mechanisms was used as a basis to group similar pixels for the spatial filter. To reduce bias caused by pixel selection and that is generated by an incorrect decomposition and classification of scattering mechanisms, an iterative refinement of decomposition and classification was used. The method was implemented on both simulated and real PolSAR images acquired from the San-Francisco area, USA and Flevoland, The Netherlands. The results were compared with other state of the art spatial filters. Results indicated that the proposed method compares favorably with other state of the art spatial filters in preserving polarimetric information, spatial details and point scatterers.

Third, we adapted the scattering mechanism based spatial filter to improve the signal to noise ratio of interferometric phase over distributed scatterers and estimate interferometric coherence matrix. Selection of the most coherent scattering mechanisms within a distributed scatterer candidate is done by applying an eigenvalue decomposition of the interferometric coherence matrix. To identify the distributed scatterer candidate for optimization we derived a phase entropy measure to be applied as a threshold. Coherent scatterers were selected by identifying scattering mechanisms in the resolution cell that interfere with the dominant scattering mechanism. Performance was evaluated on full and dual polarized SAR images acquired over Los Angeles area, USA and Groningen, The Netherlands. Number of measurement points and deformation estimates were compared with those of traditional PSI methods. Results indicate that the proposed method substantially improved the number of measurement points and the deformation estimate.

To summarize, this dissertation contributes to improving the quality of deformation measurement in natural environments from both fully polarized and partially polarized SAR images. 


\section{Samenvatting}

Metingen aan bodem-vervorming leveren waardevolle informatie op voor het begrijpen van natuurlijke gevaren zoals aard-verschuivingen, bodemdaling, aardbevingen en vulkanisme. SAR interfero-metrie vanuit satellieten biedt gedetailleerde en kost-effectieve metingen voor dit doel. De kwaliteit van deze metingen wordt echter vaak minder door decorrelatie. Hier is kwaliteit gedefinieerd als het aantal meetpunten en de nauwkeurigheid van de vervormings-metingen op basis van deze punten. De mate van decorrelatie is met name sterk in natuurlijke omgevingen waar het aantal coherente tref-elementen beperkt is. Vandaar dat het verbeteren van de kwaliteit van dergelijke metingen in natuurlijke omgevingen belangrijk is om hun bruikbaarheid te verbeteren.

Dit onderzoek richt zich op de polarimetrische diversiteit die wordt geboden door volledig en gedeeltelijk gepolariseerde SAR-beelden met als doel om het aantal meetpunten toe te laten nemen evenals de nauwkeurigheid van de schatting van de vervorming. Het is samengesteld uit drie belangrijke onderdelen. In het eerste hoofdstuk zijn de prestaties van drie polari-metrische optimalisatie methoden geanalyseerd en gevalueerd om de polarisatie fase te selecteren die het minst is benvloed door de correlatie. Dit is gedaan met behulp van enkele en dubbele fase scenarios. Deze scenarios worden toegepast op dubbele en viervoudig gepolariseerde SAR beelden verkregen uit een volledig natuurlijke omgeving in Ethiopië. Het doel is hierbij om de verbetering in de schatting van de coherentie van verschillende soorten verspreide verstrooiers te kwantificeren. On-zuiver-heid in de schattingen van de coherentie voor de verschillende optimalisatie methoden is onderzocht voor een gesimuleerd PolInSAR beeld. Vervormingen die zijn waargenomen via drie verschillende polari-metrische optimalisatie methoden zijn vergeleken met de vervormingswaarden die zijn verkregen met een in-situ GPS. De toegepaste polari-metrische optimalisatie routines zijn aanzienlijk verbeterd via deze coherentie schatting. Deze routines hebben een lagere on-zuiver-heid in vergelijking met het traditioneel gebruikte enkelvoudige polarisatie kanaal.

In het tweede hoofdstuk is een ruimtelijk filter ontwikkeld op basis van het verstrooiings-mechanisme, waarbij we gebruik hebben gemaakt van statistisch homogene pixels (SHPs). Het doel was om de signaal/ruis verhouding van de interferometrische fase te verbeteren over verdeelde verstooiers. Deze methode 
is eerst ontwikkeld om de polari-metrische consistentie matrix voor een enkel beeld te schatten. Het verbeterde de schatting van de coherentie matrix door het vermijden van het lukraak gebruik van voort-schrijdende gemiddeldes. Verbeteringen werden bereikt door het iteratief classificeren en verfijnen van de schatting van de coherentie matrix. Het verstrooiings-mechanisme is afgeleid via de Cloude-Pottier eigenwaarde-eigendecompositie techniek, waarbij de Wishart afstandsmaat werd gebruikt om het verstrooiings-mechanisme te classificeren. Classificatie van verstrooiings-mechanismen is gebruikt als basis voor het groeperen van vergelijkbare pixels voor het ruimtelijk filter. Een iteratieve verfijning van decompositie en classificatie is gebruikt om de on-zuiver-heid te verminderen die voortkomt uit pixel selectie en die door een onjuiste decompositie en classificatie van verstrooiings-mechanismen wordt veroorzaakt. De methode is toegepast op zowel gesimuleerde als echte PolSAR beelden van het gebied rond San-Francisco en beelden van Flevoland. De resultaten zijn vergeleken met andere gangbare schattingen van het ruimtelijk filter. De resultaten gaven aan dat de voorgestelde methode gunstig uitpakt in vergelijking met andere gangbare ruimtelijke filters met betrekking tot het behoud van polari-metrische informatie, ruimtelijke details en punt-verstrooiers.

In het derde hoofdstuk hebben we het ruimtelijk filter dat gebaseerd is op het verstrooings-mechanisme aangepast om de signaal/ruis verhouding van de inter-ferometrische fase te verbeteren en de interfero-metrische coherentie matrix te schatten. Een selectie is uitgevoerd van de meest coherente verstrooiings-mechanismen binnen een verdeelde verstrooiings-pixel door het toepassen van een eigenwaarde decompositie van de interfero-metrische coherentie matrix. De schatter van het ruimtelijke filter voor het verstrooiingsmechanisme is aangepast om de interfero-metrische coherentie matrix af te leiden. Om de kandidaat voor optimalisatie van verstrooiing te identificeren hebben we een maat voor de fase entropie afgeleid die als drempel moet worden toegepast. Coherente verstrooiers zijn geselecteerd door verstrooiingsmechanismen te identificeren in de resolutie cel die interfereren met het dominante verstrooiings-mechanisme. Het succes is gevalueerd op volledige en dubbele gepolariseerde SAR beelden rond Los Angeles en rond Groningen. Het aantal meetpunten en de vervormings-schattingen zijn vergeleken met die van de traditionele PSI methoden. De resultaten laten zien aan dat de voorgestelde methode het aantal meetpunten en de vervormins-schattingen aanzienlijk verbetert.

Samengevat draagt dit proefschrift bij aan de verbetering van de kwaliteit van vervormings-metingingen in natuurlijke omgevingen van zowel volledig als gedeeltelijk gepolariseerde SAR-beelden. 


\section{Acknowledgments}

When I started my studies in late 2013, I was told a $\mathrm{PhD}$ research is a singular journey, however the list that follows is an evidence to the contrary.

First and foremost, I would like to express my profound gratitude to my promoter Prof. Alfred Stein who believed and trusted in me to accomplish this $\mathrm{PhD}$ research. Without his steadfast guidance, attention and sometimes gentle prodding this thesis would not have been possible. He taught me to think as a scientist and communicate my research as such. His passion for science will always be an inspiration in my future endeavors. I would also like to express gratitude to my supervisor Dr. Valentyn Tolpekin. He was instrumental in developing my technical skills without which I would not have completed this thesis on time. His encouragement and genuine concern for my wellbeing was comforting in my worst moments. I would like to extend my appreciation to Dr. Daniele Perissin for sharing his knowledge and views on SAR interferometry to help expand mine. I'm particularly grateful for his patience to my endless line of questions on multi-temporal InSAR.

Beyond the scientific world, I would like to thank Daniel, Maurits and Rik for being good friends during my first months in the Netherlands. They made the cultural shock bearable. I would also like to thank my friends at the B.S.V. de Stottroepen: Kay, Atze, Gerlof, Dennis, Erwin, Rob, Aina and Nigel. You guys provided the prefect environment for me to escape to when I was stressed. I would also like to thank all the colleagues in EOS department especially Mengmeng, Diogo, Phillipp and their lovely partners Rita and Johanna for all the enjoyable moments that we had together. Pavel: thank you for being a friend whom I can call upon on short notice. I want to express my appreciation to my friends Eyob and Brook for their hospitality when I was in the USA as a visiting scholar. Without them my experience in the US would not have been as memorable. I would also like to thank Yuxiao, Bob and Agustin for their help and constructive comments on my research when I was at Purdue University. I also thank Francis and Jennifer for their kindness. I'm grateful to the staff of ITC especially Teresa Brefeld from EOS department, Loes Colenbrander from ITC BOOZ and Marion Pierik from financial services for helping me with different administrative matters.

Last but not least, I would like to thank my family for their unwavering 


\section{Acknowledgments}

morale support during my $\mathrm{PhD}$ research. I cannot express how grateful I am for the love I have received from them. I also want to thank my beautiful niece's for being a source of happiness and laughter whenever I call home. 


\section{Contents}

Summary

Samenvatting v v

Acknowledgments vii

$\begin{array}{ll}\text { Contents } & \text { ix }\end{array}$

1 Introduction 1

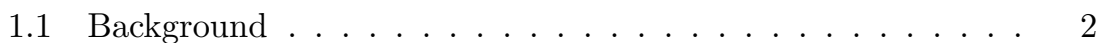

1.2 Problem statement . . . . . . . . . . . . . . . 7

1.3 Research objectives and questions . . . . . . . . . . . . 8

1.4 Thesis outline . . . . . . . . . . . . . . 9

2 Theoretical background 11

2.1 Introduction . . . . . . . . . . . . . . . . . . . . . 12

2.2 Polarimetric SAR . . . . . . . . . . . . . . 12

2.3 Polarimetric SAR and Polarimetric interferometric SAR statistics 15

2.4 Polarimetric SAR interferometry . . . . . . . . . . . . 16

2.5 PolSAR target decomposition . . . . . . . . . . . . . 21

3 Polarimetric differential SAR interferometry in an arid natural environment $\quad 23$

3.1 Introduction . . . . . . . . . . . . . . . . . . . . 25

3.2 Test area and datasets . . . . . . . . . . . . . . 26

3.3 Methodology ... . . . . . . . . . . . . . . . 27

3.4 Results .......................... 31

3.5 Discussion . . . . . . . . . . . . . . . . . . . . . . . . . . . . . . . . . .

3.6 Conclusions ....................... 40

4 Scattering Property Based Contextual PolSAR Speckle Filter

4.1 Introduction . . . . . . . . . . . . . . . . . . . . . . . . . . . 43

4.2 SAR Polarimetry . . . . . . . . . . . . . . . . 44

4.3 Scattering Property based Contextual Speckle Filtering . . . 45

4.4 PolSAR data simulation . . . . . . . . . . . . . . . . 48 
4.5 Datasets .......................... 50

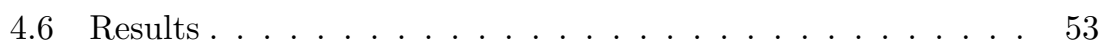

4.7 Discussion . . . . . . . . . . . . . . . . 62

4.8 Conclusions ........................ 63

5 Polarimetric scattering property based distributed scatterer PSI

5.1 Introduction . . . . . . . . . . . . . . . . . 67

5.2 Method . . . . . . . . . . . . . . . 68

5.3 Datasets ....................... . . 78

5.4 Results . . . . . . . . . . . . . . . . . . 79

5.5 Discussion and conclusion . . . . . . . . . . 86

6 Synthesis $\quad \mathbf{8 9}$

6.1 Research findings and conclusions . . . . . . . . . . . . 90

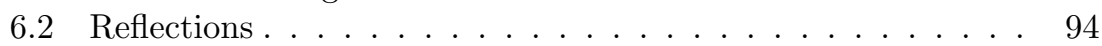

6.3 Recommendations ................. 96

$\begin{array}{ll}\text { Bibliography } & 97\end{array}$ 


\section{List of Figures}

1.1 SAR acquisition geometry ................... 3

1.2 Interferometric Synthetic Aperture RADAR acquisition geometry 5

2.1 Polarization ellipse . . . . . . . . . . . . . . . . . 14

3.1 Location map of the study area . . . . . . . . . . . . . . . . 27

3.2 Projection of LOS displacement vector into three dimensional components. . . . . . . . . . . . . . . 31

3.3 Coherence histograms for different optimization methods. . . . . 32

3.4 The coherence amplitude for different cover types. . . . . . . . . 33

3.5 Coherence loci for different optimization techniques. . . . . . . . 34

3.6 Coherence amplitude of different optimization methods as a function of the number of looks. . . . . . . . . . . . . . 36

3.7 Cumulative deformation maps derived from optimization methods. 37

3.8 Displacement time series derived from GPS and different optimization methods. . . . . . . . . . . . . . . 38

4.1 Scattering mechanisms defined on the $H / \alpha$ plane. . . . . . . . . 46

4.2 Methodology flowchart for the proposed method. . . . . . . . . . 48

4.3 Pauli images of simulated PolSAR data. . . . . . . . . . . . . . . 49

4.4 Speckle filtering results of false color composite in Pauli basis based on simulated data. . . . . . . . . . . . . . . . 52

4.5 Speckle filtering results of false color composite in Pauli basis based on simulated data . . . . . . . . . . . . . . . . . 54

4.6 Speckle filtering results of false color composite in Pauli basis based on AIRSAR airborne L band data acquired over San Francisco. . 55

4.7 Speckle filtering of a subset of the AIRSAR San Francisco bay image. . . . . . . . . . . . . . . . 56

4.8 Speckle filtering results of false color composite in Pauli basis based on AIRSAR airborne L band data acquired over Flevoland, Netherlands. . . . . . . . . . . . . . . . . . . . . 58

4.9 Intensity profile from simulated data for $\left|S_{H H}+S_{V V}\right|^{2}$ for the different speckle filters as compared with the original unfiltered data and the ground truth image. . . . . . . . . . . . . 59

4.10 Supervised Wishart classification of AIRSAR airborne L band data acquired over Flevoland, Netherlands. . . . . . . . . . . 60 
5.1 Pixel classes utilized in multi-temporal InSAR analysis . . . . . . 73

5.2 Absolute value of the polarimetric interferometric coherence matrix extracted from ALOS PALSAR data. . . . . . . . . . . . 76

5.3 Absolute value of the polarimetric interferometric coherence matrix extracted from Sentinel-1 data. . . . . . . . . . . . . 77

5.4 Location map of the two test areas. . . . . . . . . . . . . . . 78

5.5 Number of homogeneous pixels identified within $15 \times 15$ window by the classification of scattering mechanisms for the ALOS PALSAR data. . . . . . . . . . . . . . . 81

5.6 Number of homogenous pixels identified within $15 \times 15$ window by the classification of scattering mechanisms from Sentinel-1 data. 81

5.7 Comparison between unfiltered and adaptively filtered Sentinel-1 image. . . . . . . . . . . . . . . . . 82

5.8 Comparison of entropy distribution for pixels with temporal coherence greater than 0.9 for the Sentinel-1 data. . . . . . . . . . 82

5.9 Temporal coherence estimated from the (a) ALOS PALSAR dataset (b) Sentinel-1 dataset . . . . . . . . . . . . . . . . . . . . 84

5.10 The deformation rate in $\mathrm{mm}^{-1} \mathrm{r}^{-1}$ measured from the adaptively filtered ALOS PALSAR images. . . . . . . . . . . . . . . 85

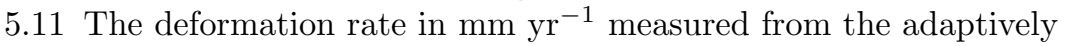
filtered Sentinel-1 images. . . . . . . . . . . . . . . 86

5.12 Displacement time series obtained from the Sentinel-1 datasets by applying different methods. . . . . . . . . . . . . . 87 


\section{List of Tables}

3.1 Acquisition parameters for the ALOS PALSAR quad and dual polarized data (co-polar and cross polar pairs) used for comparison in this chapter. . . . . . . . . . . . . 26

3.2 number of coherent pixels selected by applying coherence optimiz-

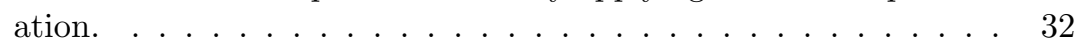

3.3 Pearson correlation coefficient $(r)$ between different optimization methods and GPS data. . . . . . . . . . . . . 37

4.1 Reference coherency matrix $(\tilde{T})$ for each simulated scattering class 51

4.2 MRF image morphology simulation parameters. . . . . . . . . . . 51

4.3 JPL/NASA AIRSAR airborne polarimetric SAR sensor acquisition parameters. ............... 53

4.4 The absolute error in $\alpha, H$ and $A$ estimates from using different speckle filtering methods for the first simulated data . . . . . . . 53

4.5 The absolute error in $\alpha, H$ and $A$ estimates from using different speckle filtering methods for the second simulated . . . . . . . . 56

4.6 Speckle filtering methods and their respective computational efficiency measured per pixel when run using an Intel Core i7 CPU with 8GB memory run on a 64 bit Linux environment. . . . . . . 57

4.7 The classification accuracy for the speckle filtered PolSAR data using different methods. . . . . . . . . . . . . . . 61

4.8 Scattering mechanism contribution after application of different speckle filtering methods. . . . . . . . . . . . . . 61

5.1 Acquisition parameters for the ALOS PALSAR quad polarized images and Sentinel-1 dual polarized images. . . . . . . . . .

5.2 List of Sentinel-1 and ALOS PALSAR data used in this chapter. In the table $\mathrm{PB}$ refers to perpendicular baseline in meters whereas TB refers to temporal baseline in days. . . . . . . . . . . . .

5.3 Number of pixels selected from the coherence matrix derived from different methods. . . . . . . . . . . . . . . 83 



\section{List of Nomenclatures}

\section{Abbreviations}

$\begin{array}{ll}\text { RADAR } & \text { Radio detection and ranging } \\ \text { SAR } & \text { Synthetic aperture RADAR } \\ \text { PolSAR } & \text { Polarimetric synthetic aperture RADAR } \\ \text { InSAR } & \text { Interferometric synthetic aperture RADAR } \\ \text { PolInSAR } & \text { Polarimetric interferometric synthetic aperture RADAR } \\ \text { DInSAR } & \text { Differential interferometric synthetic aperture RADAR } \\ \text { DEM } & \text { Digital elevation model } \\ \text { PSI } & \text { Permanent scatterer interferometry } \\ \text { PS } & \text { Permanent scatterers } \\ \text { DS } & \text { Distributed scatterers } \\ \text { GPS } & \text { Global positioning system } \\ \text { ESM } & \text { Equal scattering mechanism } \\ \text { MSM } & \text { Multiple scattering mechanisms } \\ \text { FBD } & \text { Full beam dual } \\ \text { RVoG } & \text { Random volume over ground } \\ \text { ITRF } & \text { International terrestrial reference frame } \\ \text { GNSS } & \text { Global navigation satellite system } \\ \text { IGS } & \text { International GNSS service } \\ \text { LOS } & \text { Line of sight } \\ \text { pdf } & \text { Probability density function } \\ \text { LMMSE } & \text { Local minimum mean squared error estimator } \\ \text { ENL } & \text { Equivalent number of looks } \\ \text { EP } & \text { Edge preservation } \\ \text { GP } & \text { Gradient preservation } \\ \text { IDAN } & \text { Intensity driven adaptive neighborhood } \\ \text { SNR } & \text { Signal to noise ratio } \\ \text { SHP } & \text { Statistically homogeneous pixels } \\ \text { CAESAR } & \text { Component extrAction and sElection SAR } \\ \text { QPS } & \text { Quasi permanent scatterers } \\ \text { SVD } & \text { Singular value decomposition } \\ \text { ALOS } & \text { Pdvanced land observing satellite } \\ \text { PDH } & \\ \text { PDL } & \end{array}$




\section{Symbols}

\begin{tabular}{|c|c|}
\hline$S_{X Y}$ & $\begin{array}{l}\text { Horizontal }(\mathrm{H}) \text { and vertical }(\mathrm{V}) \text { polarization states which } \\
\text { are indexed as } \mathrm{X}, \mathrm{Y}=(\mathrm{H}, \mathrm{V})\end{array}$ \\
\hline$k$ & Target scattering vector in the linear basis \\
\hline$\tilde{k}$ & Target scattering vector in the Pauli basis \\
\hline$K$ & Interferometric Target scattering vector \\
\hline$T$ & Coherency matrix \\
\hline$\Omega$ & Polarimetric interferometric coherency matrix \\
\hline$\gamma$ & Interferometric coherence \\
\hline$\phi$ & Interferometric phase \\
\hline$\mu(\omega)$ & Effective surface scattering \\
\hline G & Random Gaussian sequence \\
\hline$\theta$ & incidence angle \\
\hline$\alpha_{h}$ & Satellite azimuth vector \\
\hline$\tilde{T}$ & Reference coherence matrix \\
\hline$Z$ & Estimate of polarimetric interferometric coherency matrix \\
\hline$U$ & Matrix of eigenvectors \\
\hline$C$ & Covariance matrix \\
\hline$H$ & Scattering entropy \\
\hline$\alpha$ & Scattering mechanism \\
\hline$A$ & Anisotropy \\
\hline$I$ & Total backscattered image \\
\hline$\rho$ & Iteration termination threshold \\
\hline$\nabla$ & Sobel gradient operator \\
\hline$P$ & $\begin{array}{l}\text { Pseudo probability obtained from scattering mechanisms } \\
\text { eigenvalues }\end{array}$ \\
\hline$V_{m}$ & Coherency matrix of scattering mechanism class m \\
\hline$\omega$ & Optimal polarization state \\
\hline$\beta$ & Orientation of a target along the radar line of sight \\
\hline$\delta$ & Co-polar phase difference \\
\hline$\eta$ & Patch of homogeneous pixels \\
\hline$\Gamma$ & Coherence matrix \\
\hline$Q$ & $\begin{array}{l}\text { A matrix of eigenvectors obtained from the decomposition } \\
\text { of } \Gamma\end{array}$ \\
\hline$\Lambda$ & $\begin{array}{l}\text { A matrix of eigenvalues obtained from the decomposition } \\
\text { of } \Gamma\end{array}$ \\
\hline$\Psi$ & Phase entropy \\
\hline$c$ & Subset of eigenvectors \\
\hline$\vec{E}$ & Electric field vector \\
\hline$E_{0 x}$ & Amplitude of the electric field in the $\mathrm{x}$ axis \\
\hline$E_{0 y}$ & Amplitude of the electric field in the $y$ axis \\
\hline$\epsilon_{0}$ & Electric field permeability of vacuum \\
\hline$\mu_{0}$ & Magnetic field permeability of vacuum \\
\hline$\epsilon_{r}$ & Electric field permeability of a media \\
\hline$\mu_{r}$ & Magnetic field permeability of a media \\
\hline
\end{tabular}




$\begin{array}{ll}\nabla^{2} & \text { Laplace operator } \\ \xi & \text { Angular frequency } \\ c_{0} & \text { Speed of light } \\ \delta_{x} & \text { Constant phase term of the electric field in the } \mathrm{x} \text { axis } \\ \delta_{y} & \text { Constant phase term of the electric field in the y axis } \\ A & \text { Amplitude of the polarization ellipse } \\ \psi & \text { Orientation of the polarization ellipse } \\ \chi & \text { Ellipticity of the polarization ellipse } \\ \tau & \text { Absolute phase term of the electric field at } t_{0} \\ U_{2} & \text { Special unitary transformation matrices } \\ E_{I} & \text { Jones vector of the incident wave } \\ E_{S} & \text { Jones vector of the scattered wave } \\ \rho & \text { Complex cross correlation coefficient } \\ \psi_{N} & \text { Phase difference of the off diagonal elements of the covari- } \\ \Gamma() & \text { ance matrix } \\ { }_{2} F_{1}() & \text { Gamma function } \\ \Pi_{i j} & \text { Hypergeometric function } \\ \tilde{\omega} & \text { Whitened cross correlation matrix between images } i \text { and } j \\ U & \text { Whitened polarization state vector } \\ \lambda & \text { A matrix of eigenvectors } \\ \psi_{q} & \text { eigenvalues } \\ & \text { Cross polar phase difference }\end{array}$




\section{Introduction}




\subsection{Background}

Remote sensing can be defined as the acquisition of information without making physical contact with the target that is under observation. Depending upon the source of the measured energy, remote sensing sensors can be divided into passive and active sensors. Passive sensors use the solar energy to illuminate the target, whereas active sensors emit their own energy and capture the reflected signal. This is the case for RADAR (Radio detection and ranging) sensors. Radar sensors emit microwave pulses aimed at the earth surface in the slant direction and process the reflected signal to derive information about the distance, altitude and velocities of ground targets. These systems scan the ground scene in a slant direction which provides sensitivity in the range direction (direction of the radar path) and the azimuth direction (sensor flight direction). The azimuth resolution is determined by the transmitting antenna beam width, which is dependent on the physical antenna length. There is a limit to the applicability of these systems because of restrictions in e.g. the weight and deployment of the physical antenna.

This limitation is solved by the introduction of SAR (Synthetic aperture radar) systems. These systems illuminate the ground at regular intervals. By coherently combining the echo acquired from different positions along the trajectory of the sensors, a larger antenna size is able to be synthesized (Figure 1-1). This allows for imaging of the ground surface at a higher resolution. Radar images acquired by using this system are called SAR images. SAR systems are coherent systems that capture both the back scattered signal power (amplitude) and the number of cycles of the signal from sensor to target (phase). In SAR systems, the raw signal echo is processed into a 2-D image. This is called a single look complex (SLC) image and contains both the amplitude and the phase of the ground surface organized as pixels. Detailed information on SAR systems and SAR image formation can be found in (Elachi and Van Zyl, 2006) (Woodhouse, 2005) and (Cumming and Wong, 2005).

Traditional SAR systems operate in a single linear vertical or horizontal polarization state to transmit and receive electromagnetic waves to and from a ground target. The polarization state of an electromagnetic wave is the geometric orientation of the electric field vector. Depending upon target shape, orientation and conductivity, the backscattered wave polarization state may change (Boerner, 2006); (Krieger et al., 2005). Physical properties of a target may therefore be retrieved by utilizing multiple combinations of polarization. Polarimetric SAR (PolSAR) systems transmit and receive different combinations of polarization states. The most common types of polarization combinations are $\mathrm{HH}$ (horizontal transmit and horizontal receive), HV (horizontal transmit and vertical receive), VH (vertical transmit and horizontal receive) and VV (vertical transmit and vertical receive). In contrast with the single polarization SAR, a PolSAR system provides a matrix of scattering coefficients for each resolution cell in the image. This allows us 


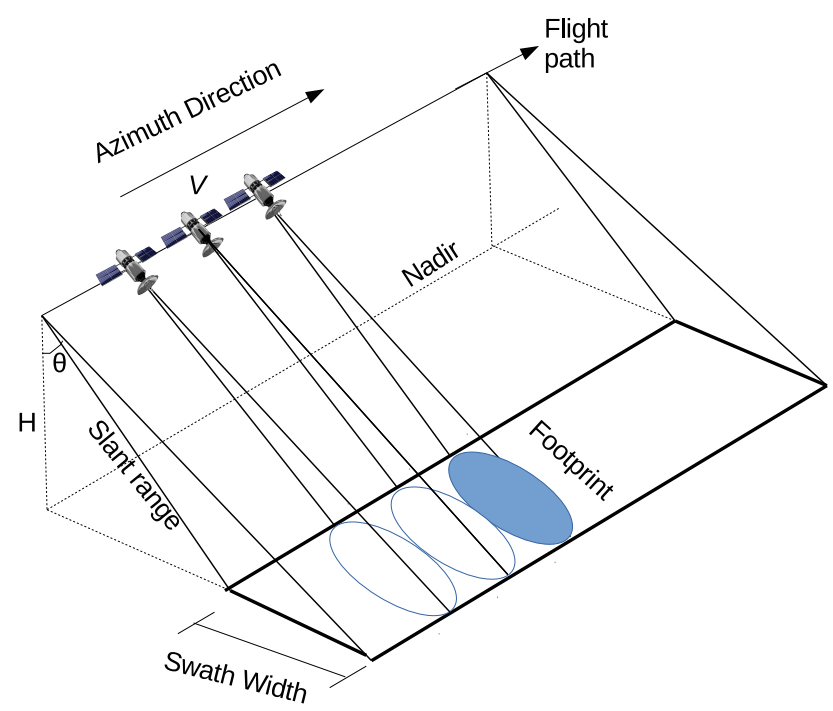

Figure 1.1: SAR acquisition geometry

to retrieve the scattering mechanism in the resolution cell and gives insight into the physical characteristics of the target. Details of PolSAR are provided in Lee and Pottier (2009).

Interferometric SAR (InSAR) techniques use the phase difference, given by the path difference between two SAR images acquired from different positions (Figure 1-2). The requirement for implementing InSAR is that the two images are coregistered and the scattering property of the scene under investigation is the same for both acquisitions. The interferogram i.e. the phase difference derived from SAR images includes 1. the phase contributions in range that results due to a reference ellipsoid, commonly referred to as a flat earth phase, 2. the phase contribution from topographic variation (topographic term), 3 . the phase contribution from change in position of the target (deformation term), 4. phase contribution introduced by the changing nature of the atmosphere in space and time and 5 . the phase noise introduced by the change in target scattering properties between the two acquisitions. For topographic height measurements, the first, the third, the fourth and the fifth term have to be removed, being undesirable phase contributions. The flat earth phase is usually estimated using the orbital information supplied with the SLC images and the reference ellipsoid parameters. The deformation phase term can be avoided by reducing the acquisition time between the two SAR images. Whereas, atmospheric and phase term due to change in scattering property can be avoided by using single pass interferometry. Once this is done, the interferometric phase consists of the topographic component only. At this stage, however, the phase values are still expressed as phase cycles modulo $2 \pi$. 
A disambiguation process termed phase unwrapping derives the continuous absolute phase values to derive absolute topographic height measurements. Detailed information on interferometric SAR processing can be found in Hanssen (2001).

As described above, a PolSAR image can be used to derive different scattering mechanisms for a ground target, whereas InSAR can be used to derive the height of a target. In polarimetric SAR interferometry (PolInSAR), PolSAR and InSAR are combined to vertically separate the different phase centers or scattering mechanisms. This has various applications like separating the height difference between tree canopy (volume scattering) and the ground surface (single bounce) allowing for the estimation of forest parameters (Neumann et al., 2010), and of ground topography estimation for vegetated regions (Lopez-Sanchez et al., 2012).

For the application of ground deformation estimation, a differential InSAR (DInSAR) processing is implemented by using the phase difference of images acquired from three or more different positions. The difference between InSAR and DInSAR is the cancellation of the topographic phase component. In DInSAR processing two types of methods can be distinguished. The first is using three or more images all coregistered to a common master. Here, one pair of images is used to estimate the topographic phase term by using the image pair with long geometric baseline and the smallest temporal baseline. This in turn is subtracted from the other image pair used to estimate the ground deformation phase term. Alternatively, an external DEM can be used to simulate the topographic phase component. This in turn can be subtracted from the interferogram to generate the deformation phase term. In this case at least two SAR images are required. Once all the undesirable phase terms have been accounted for, the wrapped phases can be unwrapped and converted to ground deformation values. In classical DInSAR the atmospheric phase term, the phase noise introduced from DEM or from orbital errors and changes in scattering properties of the target are not corrected for. Hence, classical DInSAR was only able to measure deformation resulting from large ground movements that arise from natural disasters such as earthquakes.

Various research has been undertaken to demonstrate the successful results of DInSAR in monitoring the earths surface deformation caused by subsidence, landslides, earthquakes and volcanism (Amelung et al., 1999); (Colesanti and Wasowski, 2006); (Massonnet et al., 1993); (Zebker et al., 1994); (Amelung et al., 2000). As useful as DInSAR measurements can be, there are specific limitations to the use of conventional DInSAR especially if repeat pass interferometry is used. The limitations arise from three main reasons: there is atmospheric decorrelation caused by the changing nature of the atmosphere in time, temporal decorrelation caused by decrease of signal coherence due to change to the scattering characteristics of the ground surface and geometrical decorrelation caused by a decrease of the signal coherence due to different viewing angles. Permanent scatterer interferometry (PSI) (Ferretti et al., 2001) (Berardino et al., 2002) (Colesanti et al., 2003) (Hooper et al., 


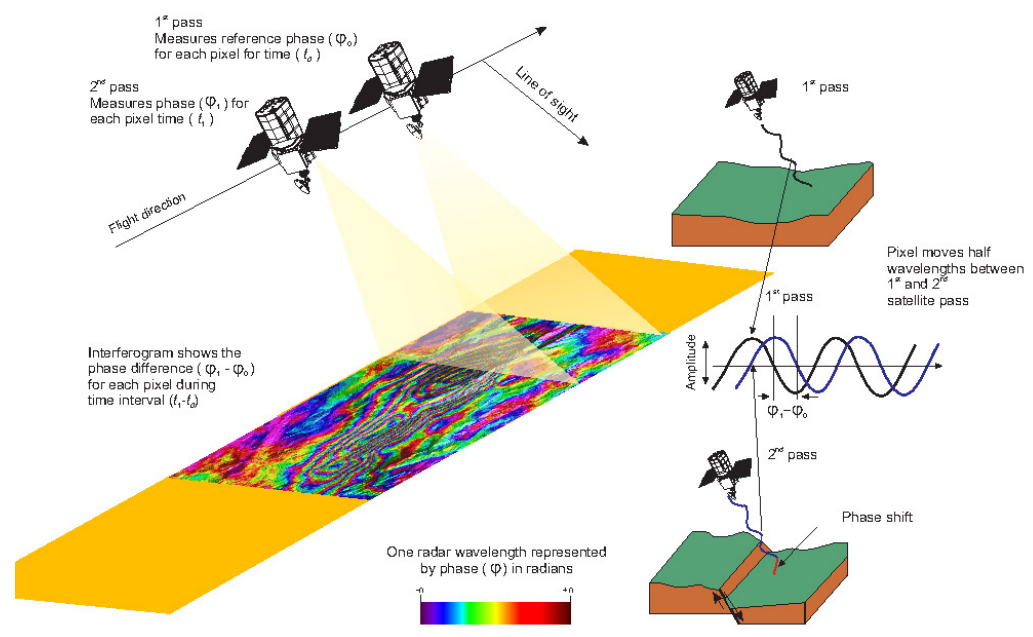

Figure 1.2: Interferometric Synthetic Aperture RADAR acquisition geometry

2004) (Kampes and Hanssen, 2004) and (Bert, 2006), a multi-temporal SAR interferometry technique, addresses both the decorrelation and atmospheric problems of classical DInSAR. It processes interferograms by selecting point like scatterers (targets with very small size relative to the resolution cell) that are coherent. The temporal decorrelation is minimal because of the stable scattering properties of the targets and atmospheric phase noise can be modeled and removed by using a large stack of interferograms. This allows for the measurement of subtle deformation events in the scale of millimeters. In contrast to classical DInSAR, where only a small number of SAR interferograms is analyzed, PSI allows to analyze a time series of interferograms.

PSI works best in urban settings with their abundant man made structures that guarantee a dense network of reliable measurement points termed persistent scatterers (PS) (Crosetto et al., 2010). In natural settings where the number of coherent targets is low, PSI selects fewer reliable PS candidates thus reducing the quality of unwrapping. To overcome this limitation, Ferretti et al. (2011) proposed SqueeSAR to enhance the capabilities of PSI by offering to exploit distributed scatterers (DS) in addition to PS. DS are extended radar targets such as agricultural fields that are more affected by geometric and temporal deccorelation. This was accomplished by establishing a statistical homogeneity test for targets to select a pixel as a DS and applying an adaptive spatial filter to improve the signal to noise ratio (SNR) in the interferograms. Moreover, it estimates the optimal differential phases from the interferometric coherence matrix by applying phase triangulation algorithm. SqueeSAR was able to significantly increase the measurement point density at the cost of resolution. Perissin and Wang (2012) sought to enhance the original PSI technique by constructing images that are most coherent with 
each other i.e. eliminating the need for each slave image to correlate uniquely with a common master. In addition, parameters are estimated by selecting a subset of the interferograms by weighing the interferograms based upon their coherences and implementing spatial filter. Both algorithms use the original PSI technique (Ferretti et al., 2001) for PS candidate selection, atmospheric phase screen and parameter estimation. Samiei-Esfahany et al. (2016) used integer least squares to estimate the interferometric phase of DS. This method allowed a formal error propagation analysis from the observations to the final estimates as it considers the mutual correlation between the differential phases.

Recent studies exploited the polarimetric information from polarimetric SAR satellites such as ALOS, TerraSAR-X and RADARSAT to increase the density of deformation measurement points. These polarimetric PSI techniques focused on identifying and relating the co-polarization phase difference between the interferograms to the scattering mechanisms of different scatterers. Different threshold values are applied to the phase difference values for selecting PS candidates (Samsonov and Tiampo, 2011). Also the techniques explored the available polarimetric space to optimize the parameters for selecting PS candidates (Navarro-Sanchez et al., 2010), (Navarro-Sanchez and Lopez-Sanchez, 2012), (Iglesias et al., 2014) and applied temporal polarimetric statistics to carry out spatially adaptive filter to DS candidates in PSI applications (Navarro-Sanchez et al., 2014). These methods were able to significantly increase the measurement point density by improving the coherence of DS just so that some of them exceed the coherence threshold in PSI analysis. They also qualitatively evaluated the improvement in measurement point density by comparing improvement in pixel number with a single polarization channel PSI.

In deformation measurement using interferometric methods the primary indicator of interferometric phase quality is coherence. To estimate coherence, commonly ensemble averaging is replaced by spatial average that defines the filter referred to as spatial filter. The estimation accuracy increases if a larger spatial window is taken, thus increasing the number of looks. Coherence, however can be severely biased especially if the estimation window consists of heterogeneous pixels with different radar signatures. To mitigate this limitation, Ferretti et al. (2011) and Jiang et al. (2015) used a statistical test to select statistically homogeneous pixels. Based upon this similarity, spatially adaptive filtering was performed thereby improving SNR of DS candidates while preserving PS candidates. By properly selecting homogeneous pixels within an estimation window, speckle can be removed from intensity images, interferometric phases can be filtered and coherence values can be properly estimated (Ferretti et al., 2011). In this regard, several studies attempted to exploit polarimetric information to identify homogeneous pixels to be used in the spatially adaptive filtering for the removal of speckle and estimation of coherence. Lee et al. (1999a) proposed to select similar pixels by using a series of edge aligned non-rectangular windows, Vasile et al. (2006) used intensity driven neighborhood region growing based upon the image intens- 
ity. Recently, Deledalle et al. (2015) used non-local means with weighted maximum likelihood estimation to reduce speckle and estimate coherence. Most methods, so far, focused on intensity statistics of the pixels without considering the scattering mechanism.

To increase the number of candidate points in a natural environment the effect of decorrelation in DS was mitigated by applying eigenvalue decomposition to the DS coherence matrix (Fornaro et al., 2015) (Cao et al., 2016). This helped to select the dominant scatterer within the resolution cell to improve the coherence estimate and interferometric phase quality. The effect of polarimetry on the selection of stable scattering mechanisms and the effect of interference of individual scattering mechanisms and their effect on coherence estimation were to be investigated to enhance the results.

\subsection{Problem statement}

The main problem statement addressed in this research is: "How can polarimetric diversity in SAR images be exploited to improve the quality of deformation measurement?". The main problem statement is divided into three specific problems which was used to formulate research questions and objectives. The specific problem statements are:

\section{Decorrelation in natural environments}

DInSAR is an established and well studied method to estimate ground deformation, however obtaining accurate measurement in a natural environment has been a challenging task due to limited number of coherent targets. The problem is even more severe for space-borne sensors with temporal baseline spanning several weeks. In this case, finding high density measurement pixels that have phase quality at a sufficient level is a challenge and is often impossible. In the literature, polarimetric optimization routines were applied to polarimetric data to determine the polarization state that is least affected by decorrelation. Significant improvement in measurement points was reported for mostly urban scenes. The performance of the polarimetric optimization methods for DInSAR applications in completely natural environments, however, has not been previously investigated. Moreover, the reliability of the different optimization methods applied on DS with multiple vertical phase centers as found in vegetated regions has not been addressed. This could be investigated to potentially improve the number of measurement points in densely vegetated regions. Moreover the bias of each coherence optimizer needs to be investigated before validating with in-situ measurements to quantify the improvement that are provided by polarimetric optimization methods.

2. Low signal to noise ratio of distributed scatterers (DS) To improve the signal to noise ratio of DS interferograms while preserving the interferometric phases of PS candidates, it is important 
that spatially adaptive filtering methods are adopted to maintain homogeneity among DS pixels used in the estimation window. This is important to minimize bias. Most literature addressed the problem of selecting statistically homogeneous pixels by considering only the intensity statistics. Scattering mechanisms, however, can provide the basis for establishing stationarity in addition to intensity statistics of the pixels for a higher accuracy in selecting homogeneous pixels. Scattering model based spatially adaptive filters that were previously proposed showed shortcomings in the estimation of scattering mechanisms. In particular, the coherence estimated from these filters was biased. An unbiased spatially adaptive filter needs to be devised that can reduce speckle in intensity images, filter the phases of DS candidates while preserving the phase of PS candidates and properly estimate coherence.

3. Low coherence of distributed scatterers (DS)

Distributed scatterers are characterized by multiple scatterers existing without a dominant scatterer inside a resolution cell. Identification of coherent scatterers and how they affect the coherence estimate of the pixel should be investigated.

By addressing these problems the quality issues that are limiting polarimetric differential interferometric analysis are expected to be mitigated.

\subsection{Research objectives and questions}

This $\mathrm{PhD}$ research focuses on exploiting the polarimetric diversity in fully and partially polarized SAR data to improve the quality of deformation measurement. The specific objectives are:

1. First Objective: To evaluate the improvements from polarization optimization methods in a natural environment.

This objective uses fully and partially polarized SAR images to apply polarimetric coherence optimization to improve the coherence of distributed scatterers. To understand the effect of polarimetric coherence optimization in vegetated areas to potentially measure deformation over vegetated regions. To investigate the bias from each optimizer by using Monte-Carlo simulation for each optimization method. Comparing the derived deformation from each optimizer to that of terresterial GPS measurement. The first objective attempts to answer the following research question: "Can polarimetric coherence optimization be used to reliably improve the quality of deformation measurement in a natural environment?"

2. Second Objective: To exploit polarimetric diversity in PolSAR data to select statistically homogeneous pixels that are suitable for estimat- 
ing the coherency matrix.

This second objective devises a spatially adaptive filter that is based upon the scattering mechanism to determine neighborhood pixel homogeneity. It focuses on devising spatially adaptive filter that is implemented for filtering speckle from intensity images. It is extended to filtering interferometric phase and coherence estimation in the third objective below. An unbiased target decomposition method is investigated and implemented. Furthermore, methods that minimize the estimation bias for the polarimetric covariance matrix are derived. The developed method is validated by comparing it with state of the art spatially adaptive filters on both simulated and real PolSAR data. In the second objective the following research question will be answered: "How can polarimetric information be used to devise spatially adaptive filter that is suitable for properly estimating coherency matrix?"

3. Third Objective: To improve the coherence estimate of DS candidates by removing incoherent scatterers.

This third objective aims to increase the density of measurement points by exploiting DS candidates in addition to PS candidates in PSI analysis. It extends the scattering mechanism based spatial adaptive filter derived in the second objective to estimate the interferometric coherence matrix. It develops a method to identify DS candidates by analyzing the interferometric phase entropy. Moreover, it applies eigenvalue decomposition to the interferometric coherence matrix to identify the most dominant scatterer within the resolution cell. The interference pattern of the secondary scatterers with the dominant scatterer within the resolution cell is investigated to remove incoherent scatterers and improve the estimation of coherence. In this objective the following research questions are answered: "Which polarimetric parameter can be used to differentiate permanent scatterers from distributed scatterers? How can the noisy scattering mechanisms within a resolution cell be eliminated to improve the interferometric coherence estimate?

\subsection{Thesis outline}

This thesis is organized into six chapters. Besides the introduction, theoretical background and synthesis chapters the three technical chapters each focus on one of the above objectives. They are all based on ISI journal articles that are published or currently undergoing review for publication.

Chapter 1 gives the general introduction to the thesis. It underscores the importance of phase quality in displacement measurement. Based on this the research objectives and research questions are introduced. 
$\square$ Chapter 2 introduces the theoretical basis for the methods used through out the dissertation.

Chapter 3 presents polarimetric DInSAR in an arid natural environment. It evaluates the reliability of polarimetric optimization techniques for displacement measurement in a completely natural environment.

Chapter 4 introduces a scattering mechanism based spatially adaptive filter suitable for removing speckle, filtering interferometric phases and estimating coherence.

Chapter 5 introduces a scattering mechanism based DS processing method for PSI applications. It derives a DS selection parameter and introduces a method that adaptively removes incoherent scatterers within the resolution cell to improve coherence estimate.

Chapter 6 summarizes the results obtained from this research and answers the research questions presented in the introduction. Reflection on the provided conclusions are presented and recommendations are also offered. 


\section{Theoretical background}

\section{Chapter summary}

This chapter presents the theoretical background of the different PolSAR and PolInSAR analysis methods used throughout this dissertation. It gives a brief mathematical representation of the different methods implemented through out the dissertation. 


\subsection{Introduction}

This chapter presents the theoretical and mathematical background for the different methods discussed in this dissertation. Section 2.2 of introduces electromagnetic wave polarization and explains the link between electromagnetic wave polarization and the different polarization channels obtained from different PolSAR sensors. The concepts described in this section are applied in Chapters 3, 4 and 5. Section 2.3 introduces the PolSAR data statistics which serves a background for Chapter 4. Section 2.4 introduces vector interferometry and describes the three polarimetric optimization methods employed in Chapter 3 whereas Section 2.5 describes the polarimetric target decomposition method used in Chapters 4 and 5 .

\subsection{Polarimetric SAR}

\subsubsection{Wave Polarization}

The fundamentals of SAR polarimetry are based upon Maxwell's equations (Stratton, 1941). It derives the wave equation for the electric field $(\vec{E})$ with the electric and magnetic permeability of vacuum as $\epsilon_{0}$ and $\mu_{0}$ respectively and the electric and magnetic permeability of the media as $\epsilon_{r}$ and $\mu_{r}$ respectively in an isotropic, homogeneous, free media as:

$\nabla^{2} \vec{E}(\vec{r}, t)+k^{2} \vec{E}(\vec{r}, t)=0$ with $k_{w}=\frac{\xi \sqrt{\epsilon_{r} \mu_{r}}}{c_{0}}$

Where, $\nabla$ is a Laplace operator, $k_{w}$ is the wave number, $\xi$ is the angular frequency and $c_{0}$ is the speed of light. The equation in (2.1) is simplified to derive the monochromatic time-space electric field as:

$\vec{E}(\vec{r}, t)=\operatorname{Re}\left\{\vec{E}_{0} e^{j \xi t}\right\}$

Here $j=\sqrt{-1}$. The electric field can be represented in the in the Cartesian coordinate system $[x, y, z]$ with the direction of propagation defined as $z$. Hence, the electric field vector as a function of time is represented in the vector form as:

$\vec{E}(z, t)=\left[\begin{array}{c}E_{0 x} \cos \left(\xi t-k_{w} z+\delta_{x}\right) \\ E_{0 y} \cos \left(\xi t-k_{w} z+\delta_{y}\right) \\ 0\end{array}\right]$

Here, $\delta_{x}$ and $\delta_{y}$ represent constant phase terms and $E_{0 x}$ and $E_{0 y}$ are the amplitude of the electric field in the $x$ and $y$ direction. Three polarization types can be defined (Lee and Pottier, 2009). 
1. Linear polarization: $\delta=\delta_{x}-\delta_{y}=0$

The electric field is a sine wave defined by a plane oriented at an angle $\psi$ with the $\mathrm{x}$ axis.

2. Circular polarization: $\delta=\delta_{x}-\delta_{y}=\frac{\pi}{2}+k \pi$ and $E_{o x}=E_{o y}$

The electric field rotates in a circular manner around the $z$ axis.

3. Elliptical polarization: otherwise

The electric field traces a helical path around the $z$ axis.

Since the 3D helical path is difficult to analyze and represent a fixed position along the direction of propagation is defined as $z=z_{0}$. In this case, the parametric relation between the $\vec{E}\left(z_{0}, t\right)$ components in time follow the equation of an ellipse, referred to as the polarization ellipse. The polarization ellipse defines the electromagnetic wave polarization.

\subsubsection{Polarization ellipse}

The shape of the polarization ellipse and the polarization state of an electromagnetic wave can be characterized by three parameters. These are the ellipse amplitude $(A)$, the ellipse orientation angle $(\psi)$ and the ellipticity $(\chi)$. $A$ is determined from the ellipse axis as:

$$
A=\sqrt{E_{0 x}^{2}+E_{0 y}^{2}} .
$$

The ellipse orientation angle $\psi \in\left[\frac{-\pi}{2}, \frac{\pi}{2}\right]$ is defined by the angle between the $x$ axis and the ellipse major axis.

$\tan 2 \psi=2 \frac{E_{0 x} E_{0 y}}{E_{0 x}^{2}-E_{0 y}^{2}} \cos \delta$.

Where $\delta=\delta_{y}-\delta_{x}$. The ellipticity of the polarization ellipse $|\chi| \in\left[0, \frac{\pi}{4}\right]$ is given as

$\tan 2 \chi=2 \frac{E_{0 x} E_{0 y}}{E_{0 x}^{2}+E_{0 y}^{2}} \sin \delta$.

The time dependent orientation of the electric field vector and its sense of rotation can be obtained from the sign of $\chi$ and the direction of propagation. To describe the wave polarization using a minimum amount of information the Jones vector is used. The Jones vector is defined from the electric field vector $\vec{E}\left(z_{0}\right)$ using the polarization ellipse characteristics $\psi$ and $\chi$ as:

$E(x, y)=A^{j \tau}\left[\begin{array}{cc}\cos \psi & -\sin \psi \\ \sin \psi & \cos \psi\end{array}\right]\left[\begin{array}{c}\cos \chi \\ j \sin \chi\end{array}\right]$ 


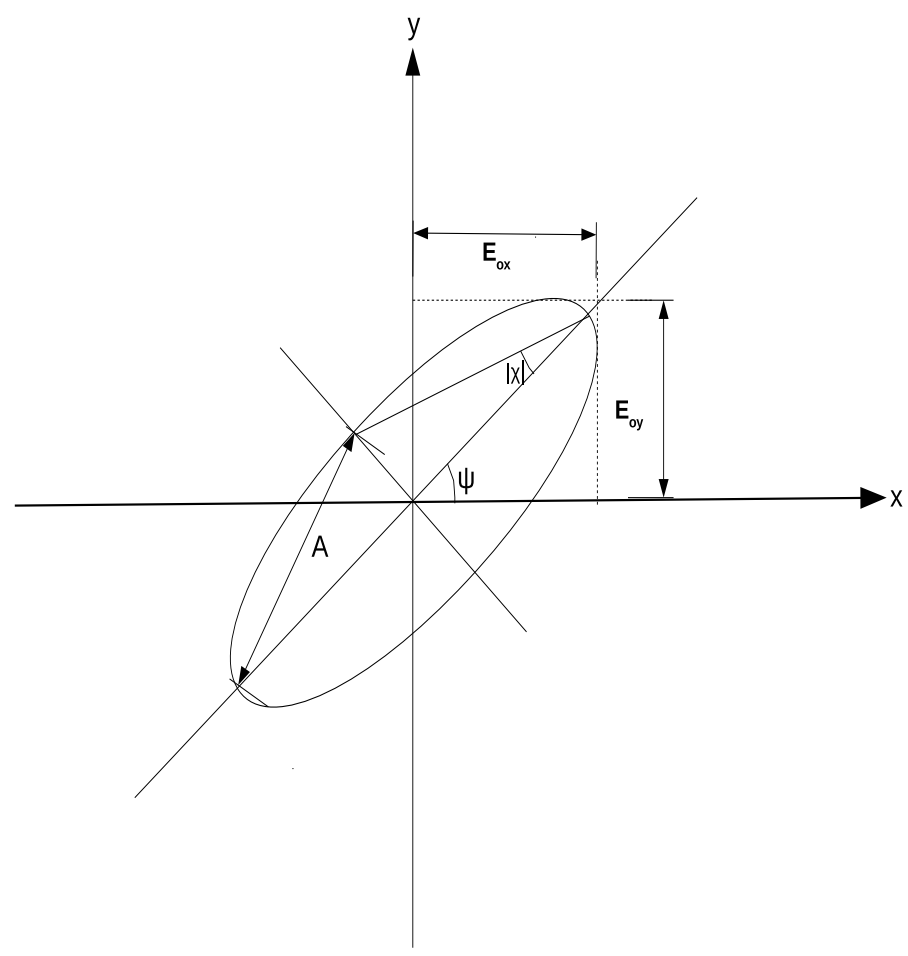

Figure 2.1: Polarization ellipse.

Here, $\tau$ represents the absolute phase term at $t_{0}$. The main advantage of using polarimetry is once a wave polarization state is defined in a basis it can be transformed to any other linear, elliptical or circular polarization basis, thereby expanding the observation space. In this regard, the Jones vector initially expressed in the Cartesian basis $x$ and $y$ can be transformed to an arbitrary polarization state $(u, p)$ by using special unitary transformation matrices $\left(U_{2}\right)$ as:

$\vec{E}(u, p)=\left[U_{2}\right]_{(x, y)} \rightarrow_{(u, p)} \vec{E}(x, y)$.

The special unitary transformation matrix $\left[U_{2}\right]$ is a $2 \times 2$ complex unitary matrix given as:

$$
\left[U_{2}\right]=\left[\begin{array}{cc}
\cos \psi & -\sin \psi \\
\sin \psi & \cos \psi
\end{array}\right]\left[\begin{array}{cc}
\cos \chi & j \sin \chi \\
j \sin \chi & \cos \chi
\end{array}\right]\left[\begin{array}{cc}
e^{j \tau} & 0 \\
0 & e^{-j \tau}
\end{array}\right]
$$


Hence, given the Jones vectors of the incident and backscattered wave $E_{I}$ and $E_{S}$ respectively, the scattering process occuring at the target is derived as:

$E_{S}=S E_{I}$

$\mathrm{S}$ is the $2 \times 2$ complex matrix called the scattering matrix or Sinclair matrix that contains the scattering coefficients of the target. Fully polarimetric SAR sensors measure the scattering matrix termed Sinclair matrix $S$. For a single pixel it equals:

$S=\left[\begin{array}{ll}S_{H H} & S_{H V} \\ S_{V H} & S_{V V}\end{array}\right]$

where the complex scattering coefficient $S_{X Y}$ indexed as $X, Y=(H, V)$ represents the horizontal $(H)$ and vertical $(V)$ polarization states, respectively.

\subsection{Polarimetric SAR and Polarimetric interferometric SAR statistics}

The statistical characteristics of the intensities, the phase and coherence are essential for PolSAR and PolInSAR (Lee and Pottier, 2009). In SAR polarimetry, $S$ is represented by the target scattering vector $k$. Assuming reciprocity i.e. $S_{H V}=S_{V H}$, the linear target scattering vector of a given scattering matrix is given in the monostatic case as:

$k=\left[\begin{array}{lll}S_{H H} & \sqrt{2} S_{H V} & S_{V V}\end{array}\right]^{\mathrm{T}}$

where $T$ designates a matrix transpose (Lee and Pottier, 2009). In distributed scatterers, the target scattering vector $k(2.12)$ can be modeled by a multivariate complex circular Gaussian distribution (Lee et al., 1994b) given as:

$p_{k}(k)=\frac{1}{\pi^{3}|C|} \exp \left(-k^{\dagger} C^{-1} k\right)$.

Here, $C=E\left\{k k^{\dagger}\right\}$ is the covariance matrix which is Hermitian positive semidefinite and $|C|$ is the determinant of the covariance matrix. For each complex element of $k$ the real and imaginary parts follow the circular Gaussian pdf. Since a mathematical expectation of the covariance matrix can't be directly calculated but estimated, a stationarity and ergodicity assumption is used to estimate the covariance matrix by using a moving window average as:

$Z=\left\langle k k^{\dagger}\right\rangle=\frac{1}{N} \sum_{i=1}^{N} k k^{\dagger}$ 
Here, $N$ is the number of looks. The probability density function of the estimated covariance matrix is given as:

$p_{Z}^{N}(Z)=\frac{N^{q N}|Z|^{n-q} \exp \left[-N \operatorname{Tr}\left(C^{-1} Z\right)\right]}{K(N, q)|C|^{N}}$,

where $K(N, q)=\pi^{0.5 q(q-1)} \Gamma(N), \ldots, \Gamma(N-q+1), \Gamma$ is the Gamma function and $q$ is the dimension of $k$. For fully polarized PolSAR data acquired in monostatic configuration $q=3$ and for single baseline PolInSAR data $q$ is 6 . The phase difference for the off diagonal elements of the covariance matrix can be estimated as:

$\psi_{N}=\arg \left(\frac{1}{N} \sum_{i=1}^{N} k(i) k(i)^{*}\right)$.

Here, ${ }^{*}$ is the complex conjugate. The phase pdf as a function of number of looks as derived by (Lee et al., 1994b) is given as:

$P_{\psi}^{(N)}\left(\psi_{N}\right)=\frac{\Gamma\left(\frac{N+1}{2}\right)\left(1-|\rho|^{2}\right)^{N} \beta}{2 \sqrt{\pi} \Gamma(N)\left(1-\beta^{2}\right)}+\frac{\left.1-|\rho|^{2}\right)^{N}}{2 \pi} F_{2}\left(N, 1 ; 0.5 ; \beta^{2}\right)$,

where, $\psi_{N} \in[-\pi, \pi], \beta=|\rho| \cos \left(\psi_{N}-\theta\right)$ and ${ }_{2} F_{1}\left(N, 1 ; 0.5 ; \beta^{2}\right)$ is the hypergeometric function. (2.17) depends only on $N$ and the complex correlation coefficient $|\rho|$ which is the coherence between the corresponding polarization channels. The peak of the distribution is assumed at $\psi_{N}=\theta$. The phase pdf becomes narrower when $N$ is increased indicating the phase quality is improved as a function of number of looks and as long as the data does not deviate from the homogeneity assumption used in deriving 2.14. This forms the basis for deriving a spatially adaptive filter presented in chapter 4, first derived to estimate the PolSAR covariance matrix and later extended to the interferometric case in chapter 5 .

\subsection{Polarimetric SAR interferometry}

Fully polarimetric SAR data can be represented with the target scattering vector $\tilde{k}$ using the Pauli basis, by assuming reciprocity (Lee and Pottier, 2009),

$\tilde{k}_{i}=\frac{1}{\sqrt{2}}\left[\begin{array}{lll}S_{H H_{i}}+S_{V V_{i}} & S_{H H_{i}}-S_{V V_{i}} & 2 S_{H V_{i}}\end{array}\right]^{\mathrm{T}}$.

In dual polarized SAR data, the target scattering vector $\tilde{k}_{i}$ reduces to: $\tilde{k_{i}}=\frac{1}{\sqrt{2}}\left[\begin{array}{ll}S_{H H_{i}}+S_{V V_{i}} & S_{H H_{i}}-S_{V V_{i}}\end{array}\right]^{T}$ in the co-polar configuration (HH VV) or $\tilde{k}_{i}=\left[\begin{array}{ll}S_{H H_{i}} & 2 S_{H V_{i}}\end{array}\right]^{T}$ in the cross polar configuration $(\mathrm{HH} \mathrm{HV})$, where 
$i \in[1,2, \ldots, n]$ is the image number for a multi-image acquisition ( $\mathrm{Ji}$ and $\mathrm{Wu}$, 2015). The polarization channels $S_{X Y}$ are indexed as $X, Y=(H, V)$ and ${ }^{\mathrm{T}}$ is the matrix transpose. The $6 \times 6$ Hermitian matrix $T_{6}$ contains both the polarimetric information contained in each image and the interferometric information of each pair.

$T_{6}=\left\langle K K^{\dagger}\right\rangle=\left[\begin{array}{ll}T_{i i} & \Omega_{i j} \\ \Omega_{i j}^{\dagger} & T_{j j}\end{array}\right]$, with $K=\left[\begin{array}{cc}{\tilde{k_{i}}}^{T} & {\tilde{k_{j}}}^{T}\end{array}\right]^{T}$.

Where $^{\dagger}$ denotes a matrix conjugate transpose and \langle\rangle represents the temporal or spatial averaging. $T_{i i}$ and $T_{j j}$ are the coherency matrices related to images $i$ and $j, \Omega_{i j}(i \neq j)$ is the polarimetric interferometric correlation matrix. In this thesis, coherency matrix represents only polarimetric information whereas coherence matrix used in Chapter 5 represents interferometric information. The polarization dependent interferometric coherence is given as:

$\gamma\left(\omega_{i}, \omega_{j}\right)=|\gamma| e^{i \phi}=\frac{\omega_{i}^{\dagger} \Omega_{i j} \omega_{j}}{\sqrt{\left(\omega_{i}^{\dagger} T_{i i} \omega_{i}\right)\left(\omega_{j}^{\dagger} T_{j j} \omega_{j}\right)}}$.

Where, $\omega$ is the complex unitary vector that is used to characterize the difference in polarized wave scattering. The scattering coefficient $\left(S_{i}\right)$ in the selected polarization state can be obtained by projecting the $\tilde{k}_{i}$ onto the selected polarization state i.e. $S_{i}=\omega_{i}^{\dagger} \tilde{k}_{i} . S_{i}$ has all the characteristics of single channel SAR image.

\subsubsection{Polarimetric Optimization}

Polarimetric optimization aims to find a unitary projection vector $\omega$ with four degrees of freedom that gives the highest interferometric coherence (2.20), i.e. the polarization state that is least affected by decorrelation. In DInSAR applications the assumption $\omega_{i}=\omega_{j}=\omega$ is made to avoid arbitrary phase components that emerge from using two different scattering phase centers for each pair of images (Colin et al., 2006). The polarimetric optimization methods described in this chapter differ on two main aspects. The first aspect is the polarimetric observation space. The second aspect is the way the polarimetric stationaity is treated in the methods. The polarization observation space is essential to explore the target response to find the polarization state that is least affected by deccorelation. For completely coherent distributed media, the polarimetric coherency matrix for each PolSAR image in a multi-baseline configuration will have similar coherency matrix if the temporal and geometric baseline is very short. However, in a repeat pass interferometric configuration especially from spaceborne sensors this is difficult to fulfill. This leads to different optimization results. The three polarimetric optimization methods (BEST, JD and ESM) are introduced in the single baseline case, whereas extension is provided as well to the multi-baseline case. 


\subsubsection{BEST Method}

This method focuses on finding the linear polarization channel that yields the highest interferometric coherence in between images (Pipia et al., 2009), (Iglesias et al., 2014). For the single baseline case it can be summarized as:

$|\gamma|=\max \left\{\left|\gamma_{h h}\right|,\left|\gamma_{h v}\right|,\left|\gamma_{v v}\right|\right\}$.

Where $\left|\gamma_{h h}\right|,\left|\gamma_{h v}\right|$ and $\left|\gamma_{v v}\right|$ are the coherence magnitude for the $H H, H V$ and $V V$ channels respectively. The interferometric phase can be obtained by taking the phase difference of the image that provided the highest coherence. For the multibaseline case, (2.21) can be modified to the polarization channel that provides the highest average coherence for all interferograms in the image stack. The BEST method is a relatively simple way to address coherence optimization as it is limited to the linear polarization state only.

\subsubsection{Equal Scattering Mechanism(ESM)}

The polarization state $\omega$ mentioned in Section 2.4 can be represented with parameters that resemble the physical attributes of radar targets. Hence, it can be parameterized as:

$\omega=\left[\begin{array}{c}\cos \alpha \\ \sin \alpha \cos \beta e^{i \delta} \\ \sin \alpha \sin \beta e^{i \psi_{q}}\end{array}\right]$

with $0 \leq \alpha \leq \pi / 2,0 \leq \beta \leq \pi,-\pi \leq \delta \leq \pi$ and $-\pi \leq \psi_{q} \leq \pi$.

In (2.22) $\alpha$ refers to the physical scattering mechanism scattering mechanism (Lee and Pottier, 2009). $\beta$ is interpreted as the orientation of the target within the radar line of sight, $\delta$ and $\psi_{q}$ are the co-polar and cross polar phase angles, respectively. Exhaustive search polarization optimization (NavarroSanchez et al., 2014) aims at finding the optimal polarization state (i.e. $\omega$ ) by numerical optimization such as conjugate gradients (Fletcher and Reeves, 1964) to find the optimum value of $\alpha, \beta, \delta$ and $\psi_{q}$. For quad polarization this technique is computationally expensive, especially for large scenes involving many images. In the dual polarization case the projection vector reduces to

$\omega=\left[\begin{array}{c}\cos \alpha \\ \sin \alpha e^{i \delta}\end{array}\right]$,

and optimization involves only two parameters. In this case, optimal parameters can be reached with reasonable computational cost. To overcome the computational burden in a fully polarized data, the ESM technique proposed by (Colin et al., 2006) can be adopted. This method uses the concept of numerical radius to solve the optimization problem. 
Let $\Pi_{i j}$ be an arbitrary $s \times s$ complex matrix. The numerical range (Stone, 1932) of this matrix is defined as:

$w\left(\Pi_{i j}\right)=\left\{\tilde{\omega}^{\dagger} \Pi_{i j} \tilde{\omega}, \tilde{\omega} \in C^{n},\|\tilde{\omega}\|=1\right\}$

Here $\tilde{\omega}$ is the polarization state in a whitened basis. Here, whitening is a linear transformation of an arbitrary vector with known covariance matrix into a vector with identity covariance matrix. The numerical range captures information about $\Pi_{i j}$ as a transformation, particularly about the eigenvalues and eigenspaces of $\Pi_{i j}$. The numerical radius is the largest absolute values of the numbers in the numerical range, i.e.

$r\left(\Pi_{i j}\right)=\max \left\{|Z|: Z \in w\left(\Pi_{i j}\right)\right\}$.

Hence, optimizing the coherence (2.20) is equivalent to calculating the numerical radius of

$\Pi_{i j}=T^{-1 / 2} \Omega_{i j} T^{-1 / 2}$.

Where $T=\frac{1}{n} \sum_{i=1}^{n} T_{i i}$ is the average coherency matrix. The square root of $T$ is defined if the coherency matrix is of full rank. Consequently, the numerical range $w\left(\Pi_{i j}\right)$ defined in $(2.24)$ is equivalent to the interferometric coherence in a whitened basis. For similar coherency matrices, the coherence values of the whitened basis and the original basis are also similar.

Hence the optimization problem in the original basis takes the form:

$\gamma_{o p t}=r\left(\Pi_{i j}\right)=\max \left|\tilde{\omega}^{\dagger} \Pi_{i j} \tilde{\omega}\right|$.

The optimal whitened projection vector can be un-whitened using

$\omega=T^{-\frac{1}{2}} \tilde{\omega}$.

To extend this technique to the multi-baseline case, the iterative technique proposed by (Neumann et al., 2008) can be adopted. This technique is based on polarimetric stationarity assumption, i.e. similar polarimetric coherency matrices at each end of the baseline. As opposed to the single baseline case, the optimization criteria in this method is the maximum sum of the whitened coherence modulus $\left(\sum\left|\tilde{\gamma}_{i j}\right|\right)$. To start the optimization routine, the modulus operation is canceled by introducing the phase shift variables $\phi_{i j} \in[-\pi, \pi], \phi_{i j}=-\phi_{i j}$ to validate the inequality:

$\max _{\tilde{\omega}} \sum_{i=1}^{n} \sum_{j=1 \neq i}^{n} \tilde{\gamma}_{i j}(\tilde{\omega}) e^{-i \phi_{i j}} \leqslant \max _{\tilde{\omega}} \sum_{i=1}^{n} \sum_{j=1 \neq i}^{n}\left|\tilde{\gamma}_{i j}(\tilde{\omega})\right|$. 
The maximum average coherence value on the right hand side is constant whereas in the left hand side it depends upon a set of $\phi$ values. It attains the value of the optimal coherence when $\phi$ assumes the optimal coherence phase. Hence, the optimization method is implemented by simultaneously searching for the optimal $\tilde{\omega} . \tilde{\omega}$ can be derived from the eigenvector associated with the highest eigenvalue $(\lambda)$ of the combined Hermitian matrix $H . H$ is defined as:

$H \tilde{\omega}=\lambda \tilde{\omega}$ where $H=\sum_{i=1}^{n} \sum_{j=1 \neq i}^{n} \Pi_{i j}(\tilde{\omega}) e^{-i \phi_{i j}}$.

The estimate for the optimal phase shifts are obtained from:

$\phi_{i j}=\arg \left(\tilde{\omega}^{\dagger} \Pi_{i j} \tilde{\omega}\right)$.

By reintroducing the $\phi_{i j}$ into (2.30) an improved estimate of $\tilde{\omega}$ is obtained. To iteratively refine these estimates, the optimization process is initialized by determining

$\phi_{i j}=\arg \left(\operatorname{Tr}\left(\Pi_{i j}\right)\right)$.

Here, Tr is the trace of a matrix. Hence, initializing from the trace corresponds to a zero eigenvalue. For every iteration an improved estimate of $\tilde{\omega}$ is estimated and that in turn leads to an improved estimate of $\phi_{i j}$. The iterative procedure is terminated if the difference between the highest eigenvalues between successive iterations is small. The optimal $\tilde{\omega}$ are converted to the original basis by using

$\omega=\frac{T^{-1 / 2} \tilde{\omega}}{\tilde{\omega}^{\dagger} T^{-1 / 2} \tilde{\omega}}$

and the optimal coherence in the original basis is then calculated from $(2.20)$.

\subsubsection{Joint Diagonalization method}

Another way to optimize the interferometric coherence is Joint diagonalization based optimization (JD) described by Ferro-Famil et al. (2009). This technique also uses the empirical symmetricalization of the whitened cross correlation matrix $\Omega_{i j}$, given as:

$\Pi_{i j}=T_{i i}^{-1 / 2} \Omega_{i j} T_{j j}^{-1 / 2}$

The whitened cross correlation matrix can also be expanded by a singular value decomposition (SVD) factorization using special unitary matrix operators as:

$\Pi_{i j}=U D U^{\dagger}, U U^{\dagger}=I$ and $\|U\|=1$ 
Here D is an arbitrary complex square matrix and $U$ is expressed as a function of three orthogonal unitary vectors in the quad polarization case and two vectors in the dual polarization case. The whitened coherence $\tilde{\gamma}$ for each of these whitened projection vectors $\tilde{\omega}$ is given by:

$\tilde{\gamma}(\tilde{\omega})=\tilde{\omega} \Pi_{i j} \tilde{\omega}$

These whitened coherences correspond to the diagonal elements of the matrix D, i.e. $\tilde{\gamma}(\tilde{\omega})=D_{i i}$. Hence, maximizing the interferometric coherence in the orthogonal direction is equivalent to maximizing the expression $\sum_{i=1}^{3}\left|D_{i i}\right|^{2}$ and minimizing $\sum_{i, j=1}^{3}\left|D_{i j}\right|^{2}$. The optimal set of orthogonal scattering mechanisms that maximizes the coherence can then be determined iteratively by using the extended Jacobi technique for simultaneous diagonalization (Cardoso and Souloumiac, 1996).

In the multi-baseline case, we follow the same technique but instead of finding the scattering mechanism that maximizes one set of interferograms we look for the optimal scattering mechanism that gives the highest average coherence in the whole stack of interferograms. In this case, as long as polarimetric stationarity is maintained, the whitened interferometric coherence is equivalent to the coherence in the original basis. Hence, when using the equal scattering mechanism, pixels that exhibit a stationary polarimetric behavior do not need an un-whitening transformation (Navarro-Sanchez et al., 2014). For pixels that exhibit a non-stationary polarimetric behavior JD results in a low coherence values. Thus by using a simple coherence threshold the points that show a non stationary behavior are discarded.

Once the optimal $\tilde{\omega}$ is determined that gives the highest average coherence for the interferogram stack, we can use (2.20) to calculate the coherence in the original basis.

\subsection{PolSAR target decomposition}

To understand the scattering properties of a radar target, an eigenvalue based target decomposition (Cloude and Pottier, 1997) is applied to a $3 \times 3$ estimate of the coherency matrix $T$. The eigenvalue decomposition of $T$ is independent on the target orientation along the radar line of sight and no a-priori information about the scene is required to derive the scattering types occurring in the scene. $T$ can be factorized into a matrix of eigenvalues and eigenvectors.

$T=U_{3} \Sigma U_{3}^{-1}=\sum_{i=1}^{3} \lambda_{i} u_{i} u_{i}^{\dagger}$, 
Where $U_{3}$ is the matrix containing three unitary orthogonal eigenvectors $U_{3}=\left[u_{1}, u_{2}, u_{3}\right]$ and $\Sigma$ is a $3 \times 3$ diagonal matrix with positive real eigenvalues $\Sigma=\left[\begin{array}{ccc}\lambda_{1} & 0 & 0 \\ 0 & \lambda_{2} & 0 \\ 0 & 0 & \lambda_{3}\end{array}\right]$. The parameterization of the eigenvector allows thee probabilistic interpretation of the scattering process. The eigenvectors can be further expanded by using physical parameters of targets as shown in (2.22) i.e.

$U_{3}=\left[\begin{array}{ccc}\cos \alpha_{1} & \cos \alpha_{2} & \cos \alpha_{3} \\ e^{i \delta_{1}} \sin \alpha_{1} \cos \beta_{1} & e^{i \delta_{2}} \sin \alpha_{2} \cos \beta_{2} & e^{i \delta_{3}} \sin \alpha_{3} \cos \beta_{3} \\ e^{i \psi_{q 1}} \sin \alpha_{1} \sin \beta_{1} & e^{i \psi_{q 2}} \sin \alpha_{2} \sin \beta_{2} & e^{i \psi_{q 3}} \sin \alpha_{3} \sin \beta_{3}\end{array}\right]$

The dominant scattering mechanism $\tilde{\alpha}$ is derived as:

$\tilde{\alpha}=\sum_{i=1}^{3} P_{i} \alpha_{i}$

where $P_{i}$ is the probability obtained from the eigenvalues $(\lambda)$ as:

$P_{i}=\frac{\lambda_{i}}{\sum_{i=1}^{3} \lambda_{i}}$

To describe the statistical disorder of the scattering mechanisms, the scattering entropy is used, given as:

$H=-\sum_{i=1}^{3} P_{i} \log _{3} P_{i}$

In eigenvalue decomposition $\tilde{\alpha}$ always assumes a value between 0 and $90^{\circ}$ and $H$ value between 0 and 1. $\tilde{\alpha}$ values that are close to 0 resemble a surface scattering, whereas $\alpha=45^{\circ}$ resembles a volume scatterer and $\tilde{\alpha}=90^{\circ}$ indicates a double bounce scattering mechanism. Entropy values close to 0 represent a deterministic scattering mechanism and an entropy value of close to 1 represents a random scattering where there is no dominant scattering mechanism. Based on $\mathrm{H}$ and $\tilde{\alpha}$, the different scattering mechanisms can be described in the alpha-entropy plane (Cloude and Pottier, 1997). 


\section{Polarimetric differential SAR interferometry in an arid natural environment}

\section{Chapter summary}

Coherence is the primary indicator of interferometric phase quality in DInSAR analysis. It is an essential concept to measure deformation in natural environments. Polarimetric optimization methods improve the coherence estimate by selecting the polarization state that is least affected by decorrelation. This chapter evaluates three polarimetric optimization methods by applying them to dual and fully polarized SAR data acquired from the ALOS satellite over a highly dynamic natural environment in Ethiopia. We first investigate their reliability by comparing the improvement in coherence estimates. Next, we evaluate the reliabiltiy of each optimizer in single and double phase center scenarios and coherence optimizer bias. Finally we compare the deformation measurement derived from each optimizer with that of a terresterial GPS measurements.

This chapter is based on: Mullissa, A. G., Tolpekin, V., Stein, A., Perissin, D., Polarimetric differential SAR interferometry in an arid natural environment. International Journal of Applied Earth Observation and Geoinformation 59:9-18, 2017. 


\begin{abstract}
Ground deformation measurements have contributed to a better understanding of the processes and mechanisms involved in natural hazards. Those include landslides, subsidence, earthquakes and volcanic eruptions. Spaceborne Differential Interferometric Synthetic Aperture RADAR (DInSAR) is a well studied technique for measuring ground deformation. Quality of deformation measurements, however, is often degraded by decorrelation. With the advent of fully polarimetric SAR satellite sensors, polarimetric optimization techniques exploiting polarimetric diversity improve the phase quality of interferograms. In this paper, we analyzed three polarimetric optimization methods to determine the optimal one for application in an arid natural environment. We considered coherence decomposition in single and double phase center scenarios. Coherence and differential phase bias associated with each optimization method has been analyzed. We compared the derived displacement values with terrestrial GPS measurements. The study shows that polarimetric optimization increases the number of coherent pixels by upto $6.89 \%$ as compared with a single polarization channel. The study concludes that polarimetric optimization coupled with DInSAR analysis yields more reliable deformation results in a low coherence region.
\end{abstract}

Keywords: Polarimetric Optimization, Differential SAR Interferometry (DInSAR), Polarimetric SAR Interferometry (PolInSAR), GPS 


\subsection{Introduction}

The advent of Interferometric Synthetic Aperture Radar (InSAR) has improved the accuracy and efficiency of surface deformation measurements. In turn, these measurements have contributed to a better understanding of the processes and mechanisms involved in natural disasters such as landslides, subsidence, earthquakes and volcanic eruptions (Massonnet et al., 1993) (Amelung et al., 2000). As useful as SAR interferometry can be, it is affected by temporal decorrelation, geometric decorrelations and atmospheric effects that all degrade interferometric phase quality (Zebker et al., 1992) (Hanssen, 2001). This in turn, governs the quality of deformation measurements in differential interferometry.

Traditionally, interferometric phase quality is assessed by means of the coherence stability (Mora et al., 2003) and the amplitude dispersion index (Ferretti et al., 2001). Recently, with the launch of fully polarimetric SAR sensors like ALOS PALSAR (Rosenqvist et al., 2007), RADARSAT-2 (Morena et al. 2004) and TERRASAR-X (Buckreuss et al., 2003), studies were undertaken that utilize the polarimetric information to find the polarization state that can provide the highest interferometric phase quality in an InSAR data stack. Polarimetric interferometric coherence optimization (Cloude and Papathanassiou, 1998) assumes either a changing dominant scattering mechanism at each end of the baseline in an interferogram or a similar scattering mechanism. Examples are the MSM (Multiple scattering mechanism) and the ESM (Equal scattering mechanism) (Colin et al., 2006). Both MSM and ESM algorithms were extended from a single baseline to include multi-baselines (Neumann et al., 2008).

Multi-baseline coherence optimization in the context of differential interferometry was introduced in Pipia et al. (2009). Navarro-Sanchez et al. (2010) and Navarro-Sanchez and Lopez-Sanchez (2012) applied polarimetric optimizations on dual polarized TERRASAR-X data to increase the number of persistent scatterer candidate points in both the amplitude dispersion index and the average coherence. Navarro-Sanchez et al. (2014) and Iglesias et al. (2014) compared polarimetric optimization methods to both the amplitude dispersion index and the average coherence in the context of permanent scatterer interferometry (PSI) using fully polarized RADARSAT-2 data. Recently, Wu et al. (2015) improved the computational efficiency of polarimetric coherence optimization by changing the four parameter optimization problem into two independent optimization problems with two parameters each. Most literature on polarimetric optimization from space-borne repeat pass configuration have applied differential interferometry to urban areas (Pipia et al., 2009) (Navarro-Sanchez et al., 2014) (Iglesias et al., 2014). Validation was done qualitatively by comparing coherence improvements with different optimization methods. As an exception Navarro-Sanchez et al. (2010) and Alipour et al. (2015) applied polarimetric optimization in a rural setting.

The novelty of the chapter is threefold. First, we apply coherence decom- 
position analysis of optimized coherences in single and double phase center scenarios for potential estimation of deformation under a vegitation canopy. Second, we combine coherence and phase bias associated with three coherence optimization methods. Third we compare the deformation as estimated from coherence optimization with terresterial GPS measurements.

The objective of this chapter was to assess, evaluate and validate polarimetric optimization for DInSAR application in an arid natural environment. The study area was located in the Ethiopian rift valley and both quad polarized and dual polarized images were applied.

\subsection{Test area and datasets}

This study focuses on the Ethiopian section of the East African rift valley. The study area has received scientific attention in the past because of its suitability for studying the role of crustal extensions and magmatism when rifting progresses to sea-floor spreading (Hammond et al., 2011). Hence, it was selected to evaluate polarimetric optimization for DInSAR applications in a natural environment. Within the study period the area has experienced dike intrusions followed by a medium level earthquake (Keir et al., 2011).

Three quad polarized ALOS PALSAR images and seven dual polarized images were used . To avoid phase aliasing, the data were oversampled twice in range. The ALOS PALSAR sensor acquires data in L-band for both quad and dual polarimetric data. It comprises $2400 \times 13000$ pixels and covers $24.3 \mathrm{~km} \times 45.5 \mathrm{~km}$ in range and azimuth directions, respectively. It is a natural environment that consists of bare surface, sparsely vegetated area, densely vegetated area and smooth sandy surface. There are several GPS sites within the vicinity of the study area (Figure 3.1), but most of the GPS points lack the temporal overlap between the image acquisition dates. The GPS stations with designation names Dabt, Datr, Da25 and 'Dayr' were used in this study.

\begin{tabular}{lcc}
\hline & Quad polarized data & Dual polarized data. \\
\hline Satellite & ALOS-PALSAR (PLY) & ALOS-PALSAR (PLY) \\
Ground resolution & $3.54 \mathrm{~m} \times 11.55 \mathrm{~m}$ & $3.54 \mathrm{~m} \times 11.55 \mathrm{~m}$ \\
Incidence angle & $23.92^{\circ}$ & $23.92^{\circ}$ \\
Orbit & Ascending & Ascending \\
Temporal baseline & 46 days & 46 days \\
Dates & $2008 / 07 / 25,2008 / 10 / 25$ and $2009 / 03 / 12$ & Same \\
Number of images & 3 & 3 \\
\hline
\end{tabular}

Table 3.1: Acquisition parameters for the ALOS PALSAR quad and dual polarized data (co-polar and cross polar pairs) used for comparison in this chapter. 


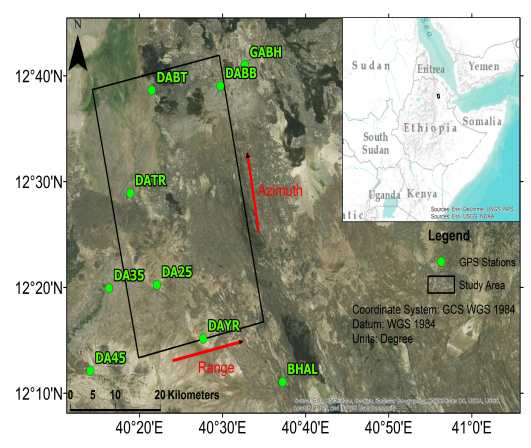

(a)

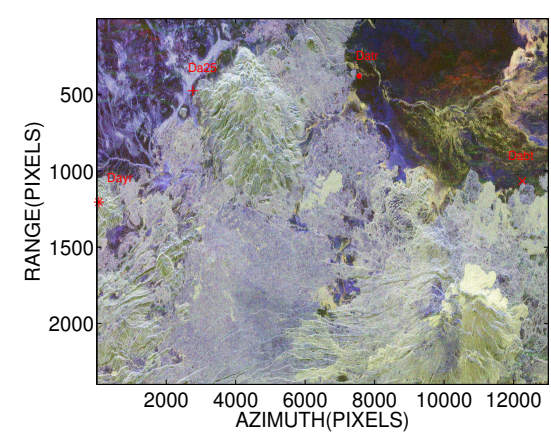

(b)

Figure 3.1: (a) Location map of the study area obtained from Google Earth. The study area, covering an area of $138.24 \mathrm{~km}^{2}$, was located in the Ethiopian section of the East African rift valley. (b) False color composite image for the study area in SAR coordinates. (Blue stands for $\left|S_{H H}+S_{V V}\right|$, green stands for $\left|2 S_{H V}\right|$ and red stands for $\left|S_{H H}-S_{V V}\right|$. The location of the GPS stations is indicated by red dots.

\subsection{Methodology}

\subsubsection{Polarimetric SAR Interferometry}

Fully polarimetric SAR data can be represented with the target scattering vector $\tilde{k}$ using the Pauli basis, by assuming reciprocity.

$\tilde{k_{i}}=\frac{1}{\sqrt{2}}\left[\begin{array}{lll}S_{H H_{i}}+S_{V V_{i}} & S_{H H_{i}}-S_{V V_{i}} & 2 S_{H V_{i}}\end{array}\right]^{T}$

In dual polarized SAR data, the target scattering vector $\tilde{k}_{i}$ reduces to: $\tilde{k}_{i}=\frac{1}{\sqrt{2}}\left[\begin{array}{ll}S_{H H_{i}}+S_{V V_{i}} & S_{H H_{i}}-S_{V V_{i}}\end{array}\right]^{T}$ in co-polar configuration (HH VV) or $\tilde{k}_{i}=\left[\begin{array}{ll}S_{H H_{i}} & 2 S_{H V_{i}}\end{array}\right]^{T}$ in cross polar configuration (HH HV), where $i \in$ $[1,2, \ldots, n]$ is the image number for a multi-image acquisition. The polarization channels $S_{X Y}$ are indexed as $X, Y=(H, V)$ and ${ }^{T}$ is the matrix transpose. The $6 \times 6$ Hermitian matrix $T$ contains both the polarimetric information contained in each image and the interferometric information of each pair.

$T=\left\langle k k^{\dagger}\right\rangle=\left[\begin{array}{cc}T_{i i} & \Omega_{i j} \\ \Omega_{i j}^{\dagger} & T_{j j}\end{array}\right]$, with $k=\left[\begin{array}{ll}\tilde{k}_{i}^{T} & \tilde{k}_{j}^{T}\end{array}\right]^{T}$.

where ${ }^{\dagger}$ denotes a matrix conjugate transpose and \langle\rangle represents spatial averaging. $T_{i i}$ and $T_{j j}$ are the coherency matrices related to images $i$ and $j, \Omega_{i j}(i \neq j)$ is the polarimetric interferometric correlation matrix and * 
denotes the matrix conjugate. The polarization dependent interferometric coherence is given as:

$\gamma\left(\omega_{i}, \omega_{j}\right)=|\gamma| e^{i \phi}=\frac{\omega_{i}^{\dagger} \Omega_{i j} \omega_{j}}{\sqrt{\left(\omega_{i}^{\dagger} T_{i i} \omega_{i}\right)\left(\omega_{j}^{\dagger} T_{j j} \omega_{j}\right)}}$.

Where $\omega$ is the complex unitary vector that is used to characterize the difference in polarized wave scattering. In DInSAR applications the assumption $\omega_{i}=\omega_{j}=\omega$ is made to avoid arbitrary phase components that emerge from using two different scattering phase centres for each pair of images. Based on (3.3), coherence optimization aims to find a unitary projection vector $\omega$ that gives the highest interferometric coherence, i.e the polarization state that is least affected by decorrelation. In this study three coherence optimization methods, BEST (Iglesias et al., 2014), Joint diagonalization based optimization (JD) (Ferro-Famil et al., 2009), (Navarro-Sanchez et al., 2014) and ESM (Colin et al., 2006), (Neumann et al., 2008) are evaluated and compared with the HH channel.

\subsubsection{Coherence Decomposition}

The interferometric coherence function can be decomposed into different decorrelation sources as:

$\gamma=e^{i \phi} \gamma_{s n r} \cdot \gamma_{t e m p} \cdot \gamma_{g e o} \cdot \gamma_{v o l}$,

where $\gamma_{s n r}$ is decorrelation due to additive noise, $\gamma_{t e m p}$ is temporal decorrelation relating to change in the scattering property of the scene, $\gamma_{g e o}$ is baseline decorrelation resulting from wave number shift due to different look angles and $\gamma_{v o l}$ is the volume decorrelation (Papathanassiou and Cloude, 2001). Note that $\gamma_{g e o}$ can be completely removed by range spectral filtering. Hence, the coherence function in a simplified form equals:

$\gamma=e^{i \phi} \gamma_{s n r} \cdot \gamma_{t e m p} \cdot \gamma_{v o l}$.

To evaluate the performance of coherence optimization in a natural environment we consider two scenarios: a resolution cell with a single phase center (surface scattering) and one with a double phase center (vegetation over ground).

\subsubsection{Single phase center}

In pure surface scattering, we assume little or no depolarization so we set $\gamma_{v o l}=1$. Hence, the polarization dependent coherence further simplifies to:

$$
\gamma(\omega)=e^{i \phi} \gamma_{s n r} \cdot \gamma_{t e m p} .
$$


Moreover, temporal decorrelation does not affect the coherence in a polarization sensitive way. Hence, it is modelled as a scalar multiplier applied to all optimization results indicating that polarization plays a direct role in minimizing the signal to noise decorrelation.

\subsubsection{Double phase center}

In a vegetated region, coherence can be modeled using a two layer scattering model like the random volume over ground (RVoG) model (Papathanassiou and Cloude, 2001). By disregarding temporal and signal to noise decorrelation the polarization dependent complex interferometric coherence is given in the RVoG model as (Cloude, 2009):

$\gamma(\omega)=e^{i \phi\left(z_{0}\right)}\left(\gamma_{v o l}+\left(\frac{\mu(\omega)}{1+\mu(\omega)}\left(1-\gamma_{v o l}\right)\right)\right)$.

Here the volume decorrelation, $\gamma_{v o l}$ is a fixed complex number independent of polarization, $\mu(\omega)$ is the polarization dependent fraction of effective surface scattering and $z_{0}$ is the ground surface. Since the volume component is complex, variation occurs in the average differential phase and coherence amplitude. Hence, as discussed in the previous section, higher coherence amplitude indicates a higher signal to noise ratio. In vegetated regions, however, it contains vegetation bias and is no longer associated with the highest phase accuracy. The coherence function is a straight line if plotted in the complex plane with one end going through $\gamma_{v o l}$ and the other through $e^{i \phi\left(z_{0}\right)}$. The optimization method that presents a phase center closest to the ground and the highest signal to noise ratio is the best optimization method in vegetated regions.

For potential estimation of displacements under a canopy we first estimate the ground phase that is corrected for vegetation bias. This is derived using the three stage RVoG inversion (Cloude and Papathanassiou, 2003). In this method, a line is fitted through the coherences in the complex plane using total least squares. The fitted line crosses the unit circle in the complex plane twice and the ground phase is located at the intersection point closest to the low phase center. By assuming absence of temporal decorrelation we can apply three-image differential interferometry on the ground phase estimated from two different pairs of images to estimate ground deformation under a vegetation canopy.

\subsubsection{Coherence Bias}

The interferometric coherence estimator, however, is biased and the magnitude of the bias depends upon the number of samples and the stationarity assumption (Touzi et al., 1999). To investigate this bias, we simulate single baseline PolInSAR data (Cloude, 2009). It starts with a known reference interferometric coherency matrix $\tilde{T}$, which is expressed using eigen decomposition as $\tilde{T}=U \Sigma U^{\dagger}$. Here $U$ is a matrix containing eigenvectors arranged in 
columns and $\Sigma$ is a diagonal matrix containing the corresponding eigenvalues $(\lambda)$.

We simulate the polarimetric interferometric target vector by first generating two independent Gaussian random sequences, $G_{a}$ and $G_{b}$, with mean $=0$ and variance $=0.5$. Those are combined into a complex series followed by scaling it by the square root of the appropriate eigenvalue (3.8). For single baseline PolInSAR data $n=6$.

$e=\left[\begin{array}{c}e_{1} \\ \cdot \\ \cdot \\ e_{n}\end{array}\right]$, with $e_{j}=\sqrt{\lambda_{j}}\left\{G_{a}(0,0.5)+i G_{b}(0,0.5)\right\}, j \in[1,2, . ., n]$.

Next, we generate $\tilde{k}(3.2)$ by collecting this series into a vector $e$ and introducing a complex correlation by multiplying this vector with the matrix of eigenvectors, i.e $\tilde{k}=U \cdot e . \tilde{k}$ follows a zero mean complex multivariate circular Gaussian probability density function.

\subsubsection{GPS Data Processing}

The GPS station data used for validation were obtained from UNAVCO's data archive interface. First, station coordinates, atmospheric delay and Earth orientation parameters were calculated using double difference phase observations. The station daily solutions were calculated by least square adjustment of double difference phase observables obtained from each station. Next, the daily solutions were combined to estimate positions and repeatabilities for each station in the ITRF 2008 reference frame (Altamimi et al., 2011). Seven international GNSS service (IGS) GPS stations designated, 'Adis', 'Mali', 'Mbar', 'Mas1', 'Mal2', 'Nurk' and 'Nklg', were utilized to tie the position calculations to the international terresterial reference frame (ITRF 2008). A maximum normalized root mean square error of 0.3 was used to ensure the quality of the observations in time. The GPS data were processed using the GAMIT/GLOBK software suite (King and Bock, 2005).

The DInSAR line of sight (LOS) displacement vector for a right looking satellite is resolved to its displacement components (Figure 3.2) (Hanssen, 2001) by

$L O S=\left[\begin{array}{lll}\cos \theta_{i n c}, & -\sin \theta_{i n c} \cos \left(\alpha_{h}-\frac{3 \pi}{2}\right), & \sin \theta_{i n c} \sin \left(\alpha_{h}-\frac{3 \pi}{2}\right)\end{array}\right]\left[\begin{array}{lll}d_{u} & d_{e} & d_{n}\end{array}\right]^{T}$.

where $\theta_{i n c}$ is the incidence angle, $\alpha_{h}$ is the satellite azimuth vector and $d_{u}$, $d_{e}$ and $d_{n}$ are the LOS displacement components in the upward, eastern and northern directions, respectively. To compare the GPS measurements with LOS displacement the same equation was used to project the three 


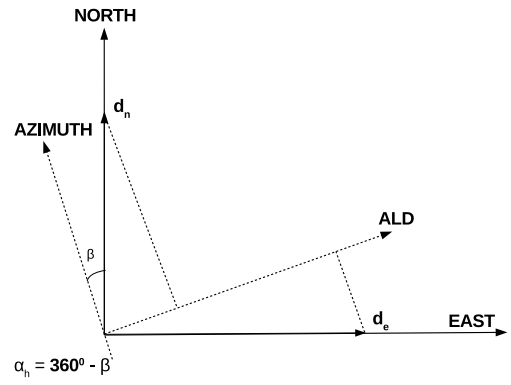

(a)

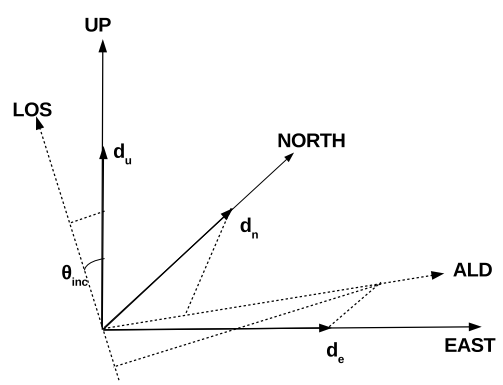

(b)

Figure 3.2: Projection of LOS vector into its 3 dimensional components $\left[\begin{array}{lll}d_{u} & d_{e} & d_{n}\end{array}\right]$. (a) Top View (b) 3-D View. ALD refers to azimuth look direction. ((Hanssen, 2001))

dimensional GPS deformation measurements to the satellite LOS displacement vector using:

$L O S_{g p s}=\left[\begin{array}{lll}\cos \theta_{i n c}, & -\sin \theta_{i n c} \cos \left(\alpha_{h}-\frac{3 \pi}{2}\right), & \sin \theta_{i n c} \sin \left(\alpha_{h}-\frac{3 \pi}{2}\right)\end{array}\right]\left[\begin{array}{ccc}G_{U} & G_{N} & G_{E}\end{array}\right]^{T}$.

The LOS vector of $\left[\begin{array}{lll}0.914, & -0.08, & 0.3963\end{array}\right]\left[\begin{array}{lll}G_{U} & G_{N} & G_{E}\end{array}\right]^{T}$ is used to project the GPS data to in the LOS direction. The absolute GPS positional measurement was converted to relative GPS deformation by taking the difference between the different acquisition dates. Once the relative three dimensional GPS deformation was converted to the satellite LOS direction, it was compared with the LOS deformation derived from each of the three optimization methods.

\subsection{Results}

\subsubsection{Coherence Optimization}

To assess the performance of coherence optimization we first compare the results of the coherence optimization for fully polarized data with coherence from the HH channel. To minimize bias when estimating the coherence, a $9 \times 9$ spatial averaging window was used to calculate the interferometric coherence values. An average coherence was obtained by averaging the estimated coherence values along the time series of interferograms. To determine the pixel phase quality a threshold of 0.7 was used. Pixels with average coherence above the threshold present a phase standard deviation of less than $5^{\circ}$ (Touzi et al., 1999). 


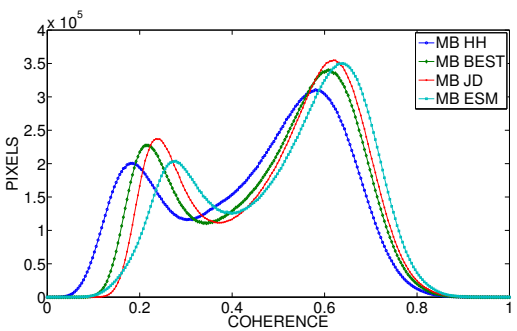

(a)

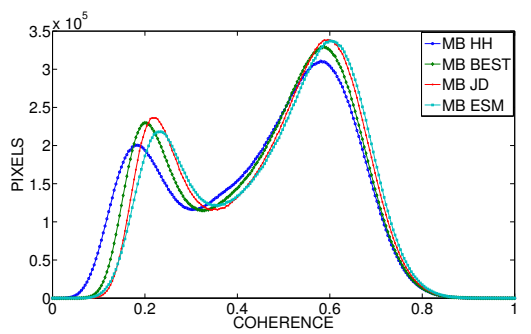

(b)

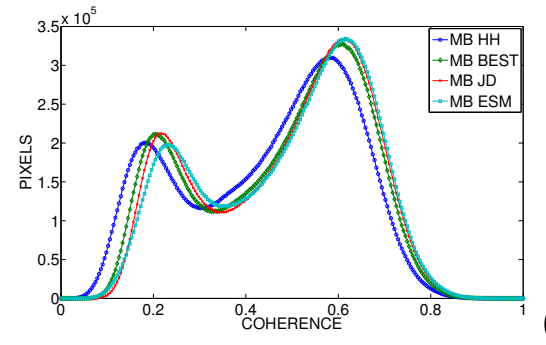

(c)

Figure 3.3: Coherence histograms for different optimization methods (a) for Quad polarized data (b) for Dual polarized data in cross polar configuration (c) for Dual polarized data in co-polar (HH VV) configuration.

\begin{tabular}{lccc}
\hline Method & QuadPol & cross polar (HH HV) & co-polar (HH VV) \\
\hline $\mathrm{t}=0.7$ & & & \\
\hline HH & $4.89 \%$ & $4.89 \%$ & $4.89 \%$ \\
BEST & $7.32 \%$ & $5.17 \%$ & $7.10 \%$ \\
JD & $8.70 \%$ & $5.94 \%$ & $8.33 \%$ \\
ESM & $11.78 \%$ & $6.77 \%$ & $8.82 \%$ \\
\hline
\end{tabular}

Table 3.2: Number of coherent pixels selected using a coherence threshold $t=0.7$ out of a total of $3.12 \times 10^{7}$ processed pixels for quad and dual polarized data in cross polar configuration ( $\mathrm{HH}$ and $\mathrm{HV}$ ) and dual polarized data in co-polar configuration (HH and VV). 


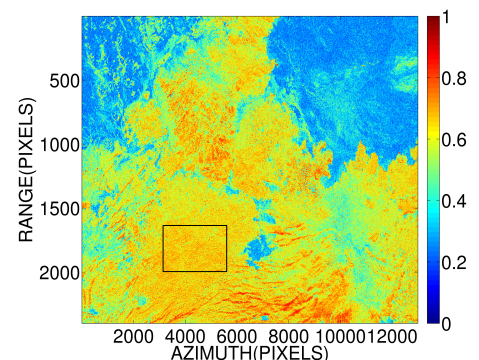

(a)

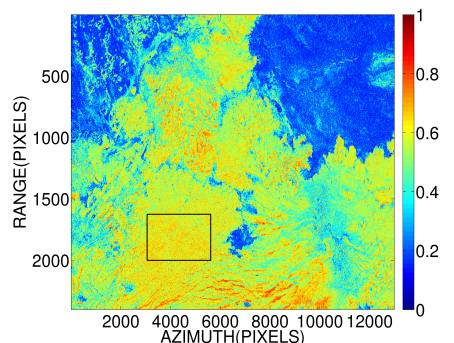

(b)

Figure 3.4: The coherence amplitude for different cover types. ESM provided improvement in all cover types but its improvement is significant in sparsely vegetated areas. (a) ESM (b) $\mathrm{HH}$

The coherence amplitude histogram for different optimization methods is shown in Figure 3.3. ESM selects the largest number of coherent pixels followed by JD and BEST (Table 3.2). Comparing the methods on their computational time, ESM requires $531 \%$ more time than HH, whereas JD requires $468 \%$ and BEST $232 \%$ more time than HH. Next, performance of coherence optimization methods is assessed for dual polarized data. To do so, dual polarization channels are synthesized from the quad polarized data. When using the co-polar channel configuration (HH VV), ESM provided the highest coherence followed by JD and BEST (Table 3.2). Clearly, the cross polar configuration gives a slightly inferior result in coherence improvement as compared to the co-polar configuration. This is because in the presence of bare surface and sparse vegetation cover, the zero polarization phase difference in the $\mathrm{HH}+\mathrm{VV}$ channel gives a higher signal to noise ratio than the HV channels. Overall, ESM achieves a higher coherence in both sparsely and densely vegetated surfaces but it achieves higher coherence improvements in bare and sparsely vegetated surfaces (Figure 3.4). These results are consistent with other studies in rural settings (Navarro-Sanchez et al., 2010) (Iglesias et al., 2015).

\subsubsection{Coherence decomposition}

The optimized complex coherences were plotted in the complex plane for bare surface and vegetated regions (Figure 3.5). In bare and sparsely vegetated surfaces, the optimized coherences followed a radial pattern that is consistent with the single phase center scattering model. As a reference, we included the low and high phase centers following the phase diversity routine described in Tabb et al. (2002). ESM showed the highest coherence modulus followed by JD, BEST and HH while exhibiting similar differential phases that indicate higher signal to noise ratio. Hence, for bare and sparsely vegetated surfaces ESM is the best optimization method. 


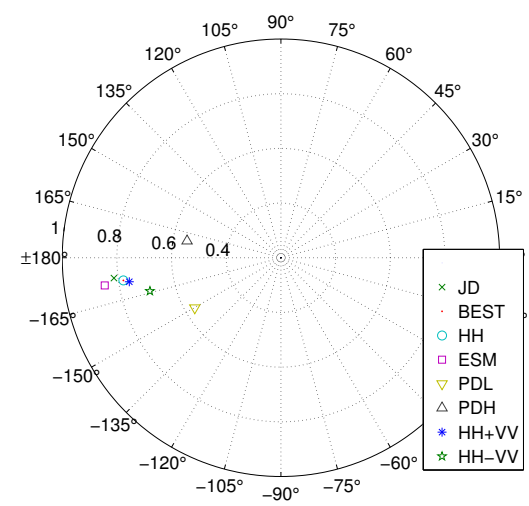

(a)

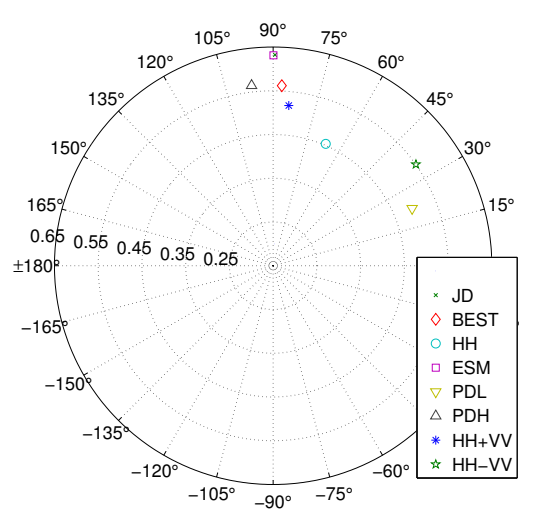

(b)

Figure 3.5: Coherence loci for different optimization techniques for (a) bare and sparsely vegetated surfaces (b) densely vegetated regions. PDL and PDH are the reference low phase center and high phase center, respectively.

In vegetated areas, ESM again provided the highest coherence amplitude but its phase center showed a random pattern. In some areas it showed a phase center close to the ground whereas in other areas it showed the highest phase center when compared with the other techniques. This is because in the RVOG model the maximum coherence can be achieved in case of a minimum surface to volume scattering $\mu(\omega)$ or of a maximum surface to volume scattering $\mu(\omega)$. Hence, the optimization method that provides the highest coherence is not necessarily the one that is closest to the ground. The HH-VV channel commonly associated with double bounce scattering generally had a lower coherence amplitude than ESM optimized coherence but it consistently yielded a phase center closer to the ground than the other optimization techniques (Figure $3.5 \mathrm{~b}$ ). In contrast, the $\mathrm{HH}+\mathrm{VV}$ channel commonly associated with surface scattering had a higher phase center when compared with both $\mathrm{HH}-\mathrm{VV}$ and $\mathrm{HH}$ channels. In vegetated areas with tree crown and trunks the HH-VV channel is dominant and achieves higher surface to volume scattering ratio with phase center very close to the ground. The HH+VV channel mostly scatters off the branches of a vegetation and doesn't achieve the highest surface to volume scattering ratio.

\subsubsection{Coherence Bias}

To determine which optimization method is least affected by coherence estimation bias, we constructed a two reference polarimetric interferometric coherency matrices $(\tilde{T})$. The first $(\tilde{T})$ matrix has identity matrix for coherency matrix in images $i$ and $j$, i.e. $T_{i i}$ and $T_{j j}=I$, and $\Omega_{i j}$ is a diagonal matrix with $\Omega_{i j}(1,1)=0.63+0.63 i, \Omega_{i j}(2,2)=0.49+0.49 i$ and $\Omega_{i j}(3,3)=0.35+0.35 i$. The second $(\tilde{T})$ matrix has $T_{i i}=\left[\begin{array}{ccc}9.4 & 0.4-0.6 i & -0.7-0.3 i \\ 0.4+0.6 i & 2.4 \\ -0.7+0.3 i & 0.2+0.02 i & 0.2-0.02 i\end{array}\right], T_{j j}=$ 


$\left[\begin{array}{ccc}9.0 & 1.1-0.6 i & -0.4-0.3 i \\ 1.1+0.6 i & 2.05 & 0.06+0.06 i \\ -0.4+0.3 i & 0.06-0.06 i & 0.7\end{array}\right]$ and $\Omega_{i j}=\left[\begin{array}{ccc}6.0-0.04 i & 0.1-0.9 i & -0.1-0.6 i \\ 0.1+0.9 i & 0.8+0.2 i & 0.1+0.2 i \\ -0.5+0.3 i & 0.09+0.09 i & 0.2+0.07 i\end{array}\right]$.

Based on these matrices, two interferometric target scattering vectors $\tilde{k}$ were simulated using the method discussed in section 3.3.3 and the mean coherence for different number of looks was compared (Figure 3.6). In both experiments ESM and BEST overestimated coherence whereas HH and JD underestimated coherence for small number of looks (less than 50 looks). For large number of looks $(>70)$, ESM coherence converged to its true value. When using the same coherency matrices at each end of the baseline, coherence converged to its true value when using a large number of looks (typically $>70$ ). When the coherency matrices deviate slightly as depicted in the second experiment, ESM coherence converged to its true value for large number of looks, but JD failed to converge to its true value even for very large number of looks $(>100)$. This indicates the sensitivity of JD to having similar coherency matrices at each end of the baseline. Moreover, if this condition is not fulfilled the coherence derived from it is always underestimated and biased. This effect is clearly shown by the differential phase error in Figure 3.6d.

The coherence estimated for $\mathrm{HH}$ and BEST depends upon the polarization channel that provides the highest coherence. If the co-polar channels provide similar coherence values then both the $\mathrm{HH}$ and BEST converge to the true coherence value with a large number of looks. If the co-polar channels provide different coherency values the $\mathrm{HH}$ and BEST coherences show large differences. In Figure 3.6c the VV channel provided significantly higher coherence than $\mathrm{HH}$ resulting in large differences between $\mathrm{HH}$ and BEST. Overall, the differential phase error is smallest for ESM followed by JD, BEST if the number of looks is large $(>70)$ and if the coherency matrices are similar at each end of the baseline (Figure 3.6b). If the coherency matrices are not similar at each end of the baseline, ESM still maintains the highest phase accuracy, whereas JD and BEST show phase errors in the estimated differential phases. With larger deviations between the coherency matrices, all optimization methods yield a low coherence and differential phases show large errors if compared to the true coherence. Hence, for the 81 looks utilized in this study and a coherence threshold of $t=0.7, \mathrm{ESM}$ is the best polarimetric optimization method with the highest phase accuracy when using fully polarimetric SAR data.

\subsubsection{Deformation measurement}

Line of sight deformation maps were generated to obtain $\mathrm{HH}, \mathrm{BEST}$, JD and ESM interferograms. The optimized interferograms were unwrapped (Chen and Zebker, 2002) and converted to total displacement values following a standard DInSAR processing flow. Since the number of quad polarized data is low it was not possible to reliably correct for atmospheric, orbital and DEM errors. Pixels with averaged coherence values $<0.7$ were masked (Figure 3.7). Furthermore, the three dimensional GPS data were projected into the satellite LOS direction for comparison using (3.10). The LOS In- 


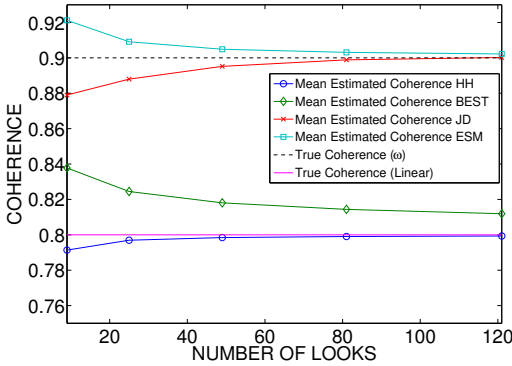

(a)

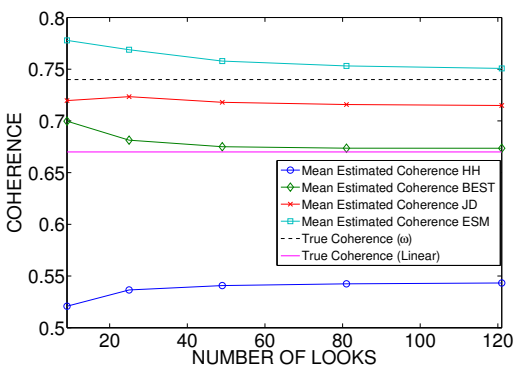

(c)

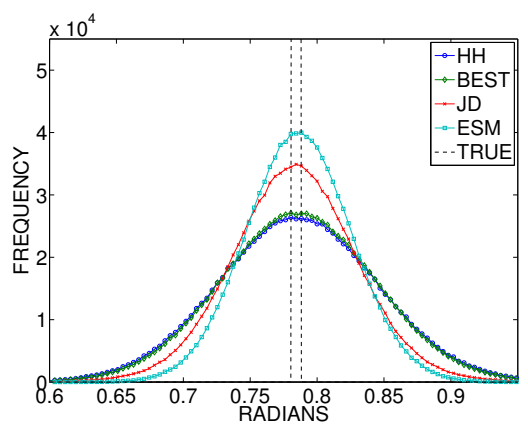

(b)

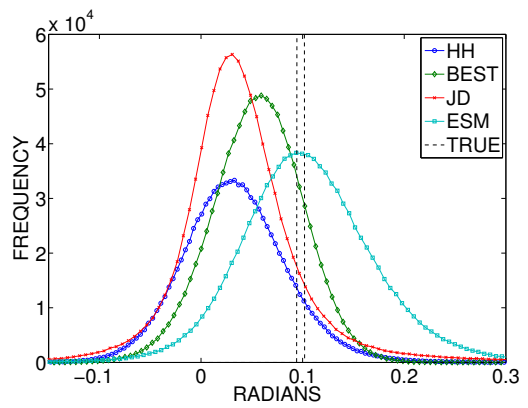

(d)

Figure 3.6: (a) Coherence amplitude (mean of $10^{6}$ realizations) of different optimization methods as a function of the number of looks, True coherence $\omega$ is the optimal coherence derived from the known coherency matrix with optimal projection vector and True coherence (Linear) is the optimal coherence calculated from the known coherency matrix using only $\mathrm{HH}, \mathrm{HV}$ and VV channels. (b) Differential phases estimated from 81 looks for different optimization methods. (c) Coherence amplitude (mean of $10^{6}$ realizations) of different optimization methods for second reference interferometric coherency matrix. (d) Differential phase estimated from 81 looks for different optimization methods.

SAR and GPS displacements were compared in terms of their deformation trend and correlation coefficient. For comparison it was not possible to find pixels that co-locate exactly with the GPS location. Hence, pixels with the nearest proximity to the GPS station and displacement standard deviation of $<0.5 \mathrm{~cm}$ was used for the displacement comparison. The terresterial GPS measurement has an uncertainty of $0.14 \mathrm{~cm}, 0.12 \mathrm{~cm}, 0.27 \mathrm{~cm}$ and $0.12 \mathrm{~cm}$ at stations Dabt, Datr, Da25 and Dayr respectively. In the displacement data, a positive sign indicates LOS displacement towards the satellite and a negative sign indicates displacement away from the satellite.

The LOS displacement measured by the optimization methods followed a decreasing trend between July 25, 2008 and March 12, 2009 for station 'Dabt' and 'Datr' (Figure 3.8). Whereas displacement at station 'Dayr' experienced 


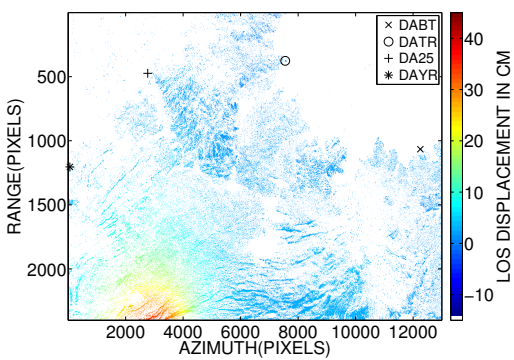

(a)

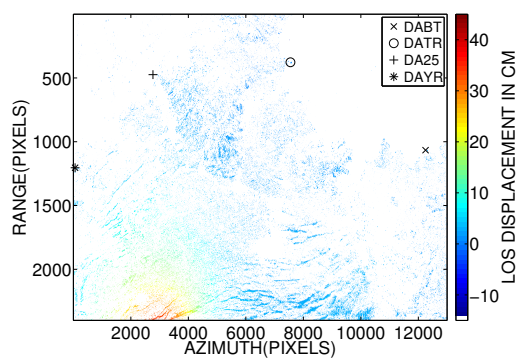

(b)

Figure 3.7: Cumulative deformation maps derived from ESM (a) and the conventional HH channel (b) inbetween July 25, 2008 and March 12, 2009. Locations of the GPS stations are shown on the map.

an increasing trend in displacement values for all methods. This was due to two earthquake events occuring on October 17, 2008 and February 11, 2009 that were induced by dike injection (Keir et al., 2011). The displacement trend is consistent with the GPS data and available literature. Figure 3.8. shows the displacement trend of ESM closely resembles the GPS data at nearly all stations. Furthermore, it showed the highest correlation coefficient $(r)$ calculated by combining the different station measurements (Table 3.3). JD displacement correlation was lower than for BEST for quad and dual polarized data (HHHV) and for some stations displacement measurement least resembled the GPS data and other optimization methods (Figure $3.8 \mathrm{~h}$ and $3.8 \mathrm{k}$ ). This is consistent with the phase bias analysis presented in section 3.4.3. Atmospheric, orbital and DEM errors were not corrected and hence displacement estimates deviated from $0.25 \mathrm{~cm}$ to $1 \mathrm{~cm}$ from GPS measurements. Overall, ESM provided the highest accuracy in displacement estimation followed by JD, BEST and HH. In future work, the analysis should be extended to multi-temporal InSAR analysis (PSI) with more GPS stations, as this may improve the significance of the work.

\begin{tabular}{lccc}
\hline Method & QuadPol & cross polar (HH HV) & co-polar (HH VV) \\
\hline HH & 0.86 & 0.86 & 0.86 \\
BEST & 0.88 & 0.91 & 0.90 \\
JD & 0.87 & 0.89 & 0.92 \\
ESM & 0.96 & 0.93 & 0.96 \\
\hline
\end{tabular}

Table 3.3: Pearson correlation coefficient $(r)$ between different optimization methods and GPS data. Since a limited number of images are used to derive the displacement values, different stations are combined to derive the correlation coefficients. Displacement values corresponding with missing GPS data are not included in the calculation. 


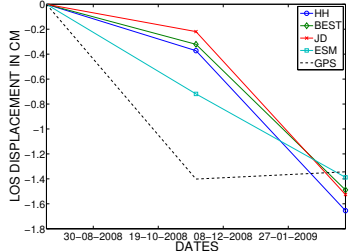

(a)

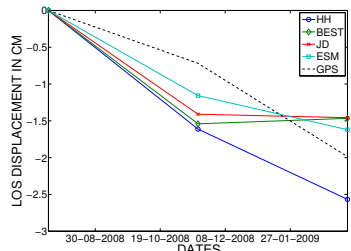

(d)

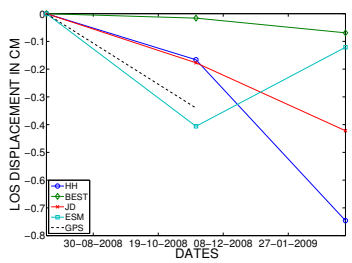

(g)

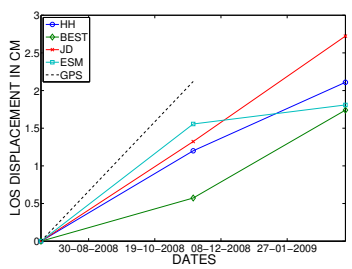

(j)

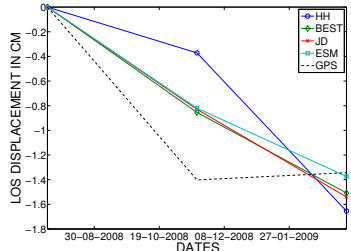

(b)

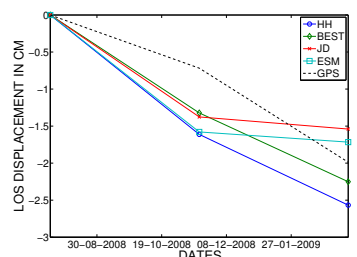

(e)

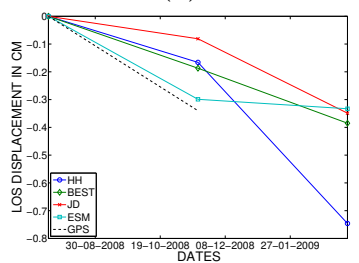

(h)

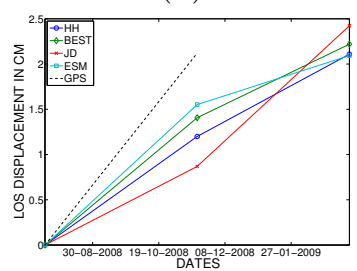

$(\mathrm{k})$

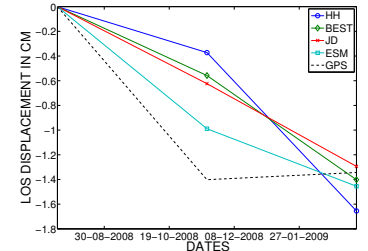

(c)

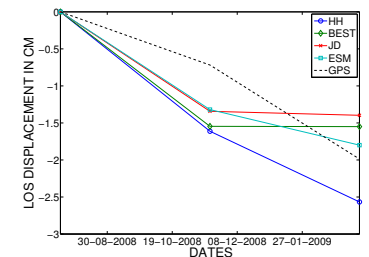

(f)

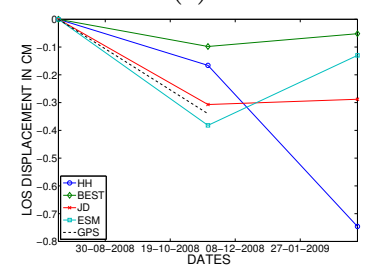

(i)

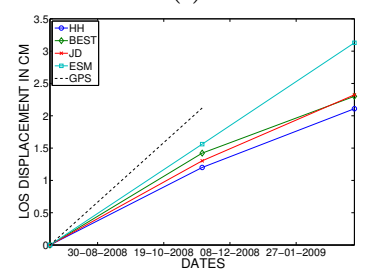

(l)

Figure 3.8: Displacement time series derived from GPS and different optimization methods. Lines are included in the plot for illustration purposes. Rows display the stations Dabt, Datr, Da25 and Dayr, whereas the columns show Quad polarized, cross polar (HH HV) and co-polar (HH VV), respectively. 


\subsection{Discussion}

The study showed that ESM achieved the largest improvement in both bare and sparsely vegetated surfaces as compared to JD, BEST and conventional $\mathrm{HH}$ channel. ESM, however, is formulated based on the hypothesis of polarimetric stationarity. When this hypothesis is not fulfilled, ESM results in low average coherence during optimization. It should be noted that even in this scenario it provides higher coherence than JD, BEST or HH (Figure 3.3 ). However, it will most likely result in a low average coherence in the interferogram stack that is below the coherence threshold. Hence, coherence optimization has little additional value. In densely vegetated regions the coherency matrices at each end of the baseline differ significantly and therefore result in a relatively low differential phase accuracy and in a random phase center location. Hence, in densely vegetation regions a polarization state that provides a phase center that is close to the ground surface should be used to derive displacement values. In this regard, HH-VV provides a phase center that is located closer to the ground surface as shown in Section 3.4.2 even-though it may select fewer coherent pixels than the optimization methods discussed in this chapter. If trunk structure is absent within the vegetation canopy a coherence loci analysis should be performed to select the polarization state that selects a phase center that is closest to the ground surface. Moreover, to improve the optimization results, polarimetric stationarity can be mitigated by modeling the correlation between images based on their temporal and geometric baselines.

By using the RVoG model inversion it was possible to obtain the ground phase by removing the vegetation bias. The ground phase is useful to obtain geophysical parameters such as ground topography under vegetation canopy. In theory, the ground topography extracted from RVoG inversion also includes deformation signals. Therefore it is possible to estimate ground deformation under a forest canopy by removing the ground topography using repeat pass space-borne interferometry. In this study, due to the long temporal baseline, the signal was affected by temporal decorrelation. Hence, RVoG inversion resulted in a low coherence which could not be reliably unwrapped to derive the ground displacement under a canopy. If temporal decorrelation could be mitigated, however, repeat pass polarimetric differential SAR interferometry under a vegetation canopy is feasible.

Coherence optimization in the context of DInSAR in a natural terrain has practical uses in determining hazards from volcanic eruptions and earthquakes. In densely vegetated regions, even though classical DInSAR cannot be applied because of temporal decorrelation, selective processing of coherent pixels with stable scattering mechanism in multi-temporal InSAR context will yield superior results as compared to conventional single polarization channels. In multi-temporal InSAR analysis pixels are selected for parameter estimation based on their quality. Thus coherence optimization increases the number of pixel candidates that are available for processing because the candidate points are denser. Consequently, denser points will give a 
more robust model fitting because of more individual connections between candidate points making the deformation measurements more reliable.

\subsection{Conclusions}

We conclude that ESM provided the highest phase quality improvement, followed by JD and BEST when optimizing quad-pol data sets in bare and sparsely vegetated regions. In densely vegetated regions ESM attained the highest signal to noise ratio whereas the phase center of $\mathrm{HH}-\mathrm{VV}$ polarization channel was closest to the ground surface. In addition, ESM was least affected by phase bias as compared to $\mathrm{HH}$ polarization in bare and sparsely vegetated regions. A similar trend was observed when optimizing dual polarized data sets: the co-polar configuration performed better than the cross polar configuration, despite reduced data dimensions. Finally, ESM deformation results showed the highest correlation with the continuous GPS measurements, even though DEM, orbital and atmospheric phase errors were not corrected. Hence, polarimetric optimization coupled with DInSAR analysis yielded more reliable deformation results as compared with $\mathrm{HH}$ polarization in a low coherence region. 


\section{Scattering Property Based Contextual PolSAR Speckle Filter}

\section{Chapter summary}

Coherence is a phase quality indicator in InSAR analysis. To estimate coherence, commonly spatial average commonly referred to as spatial filter is used. The estimation accuracy increases if a larger spatial window is taken, thus increasing the number of looks. Coherence, however can be biased especially if the estimation window consists of heterogeneous pixels with different radar signatures. The most common approach to do so is average pixels that share the same property within a neighborhood window. This procedure is called spatially adaptive filtering. Chapter 4 derives a scattering mechanism based spatially adaptive filter that is suitable for filtering speckle in PolSAR data and estimate coherence in PolInSAR data stack. Reliability of the derived spatially adaptive filter is evaluated on simulated and real PolSAR data.

This chapter is based on: Mullissa, A. G., Tolpekin, V., Stein, A., Scattering property based contextual PolSAR speckle filter. International Journal of Applied Earth Observation and Geoinformation 63: 78-89, 2017. 


\section{Abstract}

Reliability of the scattering model based polarimetric SAR (PolSAR) speckle filter depends upon the accurate decomposition and classification of the scattering mechanisms. This chapter presents an improved scattering property based contextual speckle filter based upon an iterative classification of the scattering mechanisms. It applies a Cloude-Pottier eigenvalue-eigenvector decomposition and a fuzzy $H / \alpha$ classification to determine the scattering mechanisms on a pre-estimate of the coherency matrix. The $H / \alpha$ classification identifies pixels with homogeneous scattering properties. A coarse pixel selection rule groups pixels that are either single bounce, double bounce or volume scatterers. A fine pixel selection rule is applied to pixels within each canonical scattering mechanism. We filter the PolSAR data and depending on the type of image scene (urban or rural) use either the coarse or fine pixel selection rule. Iterative refinement of the Wishart $H / \alpha$ classification reduces the speckle in the PolSAR data. Effectiveness of this new filter is demonstrated by using both simulated and real PolSAR data. It is compared with the refined Lee filter, the scattering model based filter and the non-local means filter. The study concludes that the proposed filter compares favorably with other polarimetric speckle filters in preserving polarimetric information, point scatterers and subtle features in PolSAR data.

Keywords: Polarimetric Synthetic Aperture RADAR (PolSAR), Speckle filtering 


\subsection{Introduction}

Polarimetric synthetic aperture radar (PolSAR) and polarimetric interferometric SAR (PolInSAR) are useful to estimate physical parameters at the earth surface. The SAR signal is commonly affected by speckle, arising from the coherent superposition of a number of independent scatterers within each resolution cell. If the size of the resolution cell is much larger than the imaging wavelength there will be interference between individual scattering producing speckle. For polarimetric image analysis purposes, this is characterized as noise because of the complexity of the scattering process. In the presence of speckle the polarimetric response is best taken as a random value. To determine its probability density distribution, second order statistics represented by the polarimetric covariance matrix have to be estimated.

PolSAR speckle filtering and information extraction rely on the applied stochastic model for the PolSAR data. In homogeneous and stationary media, PolSAR data can be characterized by a zero mean multivariate circular Gaussian pdf (Lee et al., 1994b). The simplest method proposed for reducing speckle is the boxcar filter (Lee, 1980) that estimates the covariance matrix with a moving average operation. The boxcar filter is effective in removing speckle in homogeneous regions at the expense of loss in resolution. To overcome this loss, Lee et al. (1999b) proposed to select similar pixels by using a series of edge aligned non-rectangular windows, Vasile et al. (2006) used intensity driven neighborhood region growing based on the image intensity, Lee et al. (2006) proposed selecting similar neighboring pixels based on scattering characteristics and Lopez-Martinez and Fabregas (2008) suggested an adaptive speckle reduction based on additive and multiplicative noise model. Further, Deledalle et al. (2015) used non-local means with weighted maximum likelihood estimation to reduce speckle, Chen et al. (2011) developed a statistical pre-test method to select homogeneous patches and Zhong et al. (2014) used a statistical testing to establish pixel similarity between two patches that follow a complex Wishart distribution. Most methods, however, focused on pixel intensity statistics without considering pixel scattering properties. An exception was Lee et al. (2006) who considered pixel statistics and polarimetric scattering mechanisms for speckle filtering.

The scattering model based (SMB) speckle filtering method (Lee et al., 2006) applied the Freeman-Durden model based decomposition (Freeman and Durden, 1998) to determine the dominant scattering mechanism power on a pixel by pixel basis. Unsupervised classification is initialized by grouping pixels into clusters for each canonical scattering mechanism. These are merged into classes for each scattering mechanism using a Wishart merge distance measure. This method preserves the dominant scattering mechanisms of each pixel. The following limitations require compensation. First, the Freeman-Durden model based decomposition tends to overestimate the volume scattering component in urban settings (Yajima et al., 2008) (Yamaguchi et al., 2011). It assumes that all cross polarized return is due to vegetation. Rough surfaces and local topography, however, can also cause depolarization resulting in a higher cross 
polarization return in SAR data. Hence, to achieve improved decomposition results from the Freeman-Durden decomposition it is important to correct for the orientation angle. This increases processing time for speckle reduction. Second, clustering the scattering classes into classes for each scattering mechanism implies that pixels that are filtered together belong to the same scattering mechanism. This clustering of pixels is efficient in case of relatively accurate decomposition results and classification of scattering mechanisms. If a pixel is dominated by noise or if its amplitude level is near the noise floor, decomposition may lead to less accurate results (Wang and Davis, 1997). Hence, subsequent filtering based upon this method of selection will lead to less accurate results. Third, in Lee et al. (2006) the Freeman-Durden decomposition is initialized with a $3 \times 3$ pre-estimate of the covariance matrix to estimate the three main scattering mechanisms: single bounce, double bounce and volume. This decomposition is inherently biased when using a small number of pixel samples. Its quantification is in-sufficiently addressed in the literature, although, Lee et al. (2008) evaluated the bias introduced in target decomposition and concluded that parameters of the scattering mechanism are best estimated using at least a $9 \times 9$ multi-looking window.

The objective of this chapter is to improve the existing SMB speckle filtering method for PolSAR data. It builds upon SMB filtering by addressing the specific limitations listed above. The proposed method uses the Cloude-Pottier eigenvalue-eigenvector decomposition of the coherency matrix to reduce bias in the target decomposition. Both coarse and fine pixel selection are included to minimize the pixel selection error. It also minimizes the scattering mechanism decomposition and classification bias by iteratively increasing the number of looks included in estimating the scattering mechanism and refining the scattering mechanism classification. The method is applied on both simulated data and real PolSAR data acquired from the AIRSAR airborne sensor acquired over Flevoland, The Netherlands and San Franscico, USA.

\subsection{SAR Polarimetry}

Fully polarimetric SAR sensors measure the scattering matrix (Sinclair matrix) $S$. For a single pixel it equals:

$S=\left[\begin{array}{ll}S_{H H} & S_{H V} \\ S_{V H} & S_{V V}\end{array}\right]$,

where the complex scattering coefficient $S_{X Y}$ indexed as $X, Y=(H, V)$ represents the horizontal $(H)$ and vertical $(V)$ polarization states. In SAR polarimetry, $S$ is represented by the target scattering vector $k$. Assuming reciprocity i.e $S_{H V}=S_{V H}$, the linear target scattering vector of a given scattering matrix is given in the monostatic case as:

$k=\left[\begin{array}{lll}S_{H H} & \sqrt{2} S_{H V} & S_{V V}\end{array}\right]^{T}$ 
where $T$ designates a matrix transpose (Lee and Pottier, 2009).

For deterministic point scatterers $k$ fully describes the scattering process. For distributed scatterers, however, $k$ displays a random property and is modeled (Lee et al., 1994b) by a multivariate complex circular Gaussian probability density function :

$p_{k}(k)=\frac{1}{\pi^{3}|C|} \exp \left(-k^{\dagger} C^{-1} k\right)$

Here $C=E\left\{k k^{\dagger}\right\}$ is the covariance matrix, $|C|$ is the determinant and $\dagger$ denotes the complex conjugate transpose. Assuming stationarity and ergodicity in the neighborhood of a pixel, an estimate of the covariance matrix is obtained by:

$Z=\left\langle k k^{\dagger}\right\rangle=\frac{1}{n} \sum_{i=1}^{n} k k^{\dagger}$

where $n$ is the number of looks used to estimate $Z$. The distribution of $Z$ is modeled by the complex Wishart probability density function (Goodman, 1963). Note that the covariance matrix can be transformed into the coherency matrix $T$

$T=\left(S U_{3}\right) C\left(S U_{3}\right)^{-1}$

where $S U_{3}=\frac{1}{\sqrt{2}}\left[\begin{array}{ccc}1 & 0 & 1 \\ 1 & 0 & -1 \\ 0 & \sqrt{2} & 0\end{array}\right]$ is a unitary transformation matrix (Lee and Pottier, 2009).

\subsection{Scattering Property based Contextual Speckle Filtering}

\subsubsection{Scattering mechanism classification}

The Cloude-Pottier eigenvalue-eigenvector decomposition (Lee et al., 1999a) is applied to correct for bias caused by the Freeman-Durden decomposition. It is selected because it is able to derive the scattering mechanism $(\alpha)$ and scattering entropy $(H)$ without being affected by differences in the orientation angle and no a-priori scene information is required to derive the scattering mechanism.

Based on $H$ and $\alpha$ values, pixels can be classified into their respective scattering classes. The $H / \alpha$ plane (Figure 4.1b), however, reveals continuously distributed $H / \alpha$ values, whereas the class boundaries in Figure 4.1a are crisp. Such ambiguity presents a problem of defining class mixtures near the boundaries. To resolve it we implemented a fuzzy $H / \alpha$ classification 


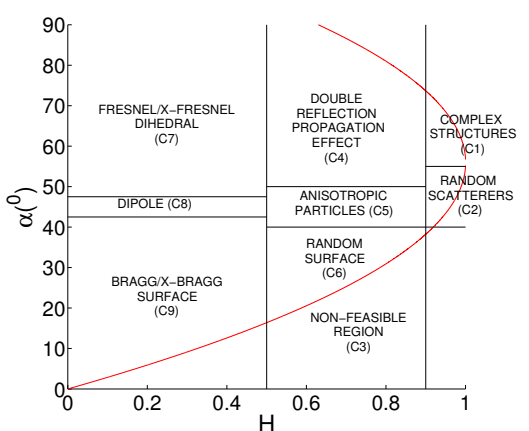

(a)

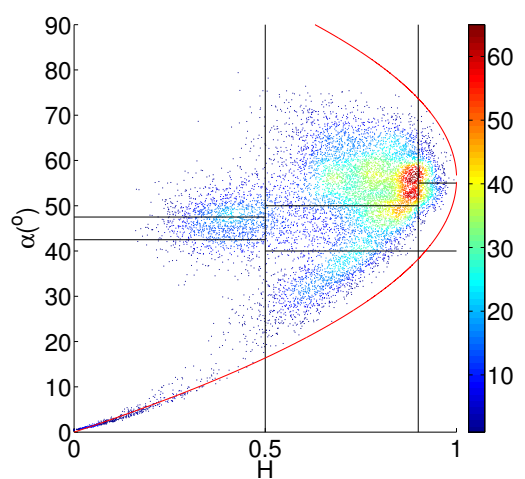

(b)

Figure 4.1: (a) The $H / \alpha$ plane showing different scattering properties, as defined in Cloude and Pottier (1997) and Cloude and Pottier (1996). (b) The $H / \alpha$ density plot derived from a simulated PolSAR data. The color scale indicates the number of pixels per data point.

(Park and Moon, 2007) for the first filtering. This is followed by the Wishart distance classification in the next iterations (Lee et al., 1999a).

\subsubsection{Speckle Filtering}

We carry out the speckle reduction in two steps. First, we derive the rules that guide the speckle filter. This step is important to select homogenous pixels discussed in the introduction section. Second, we iteratively refine the scattering mechanism classification to reduce the estimation of scattering mechanism and classification bias that arises from initializing from a small number of looks while improving the filtering result.

\subsubsection{Rule derivation}

The fuzzy $H / \alpha$ classification output is defined in the $H / \alpha$ plane, typically resulting in eight scattering classes. The application of the term 'classes' in this context doesn't imply thematic classes. A physical interpretation of the classified output is detailed in Lee and Pottier (2009). Based upon this interpretation, coarse and fine pixel selection are introduced. Coarse pixel selection groups the scattering classes into single bounce, double bounce and volume scattering mechanisms. The canonical scattering mechanisms are derived from the fuzzy $H / \alpha$ classification output using the procedure described in Ferro-Famil et al. (2002). Hence, pixels grouped as single bounce scattering are un-filtered from pixels grouped as double bounce or volume scattering. Similarly, pixels grouped as double bounce and volume scattering remain separate from other groups to preserve the dominant scattering mechanism of the pixel. The brightest pixels within the single bounce and 
double bounce group larger than a 9 pixel patch are un-filtered i.e they keep their original pixel values in order to remove isolated deterministic point scatterers. Fine pixel selection does not group the scattering classes to their main scattering mechanisms, i.e pixels from the eight scattering types remain separate from other classes. Hence, they are filtered separately even if pixels share the same canonical scattering mechanism. The brightest pixels from Class 1 (deterministic dihedral scattering) and Class 3 (Bragg scattering) larger than a 9 pixel patch remain un-filtered, whereas the other scattering types are filtered from pixels within the same scattering class.

Depending upon the image scene environment (urban or rural) we can either use coarse selection, fine selection or a combination of the two to obtain the optimal output. For pixels thus selected, we use the local minimum mean squared error estimator (LMMSE) that is commonly used to estimate the filtered coherency matrix (Lee et al., 1999b). Depending on the applied selection rule, pixels within the filter window belonging to the same scattering type are therefore filtered as:

$\tilde{T}=\langle T\rangle+b(T-\langle T\rangle)$.

Here $\tilde{T}$ is the result of speckle filtering and $\langle T\rangle$ is the average single look coherency matrix of pixels with the same scattering class as defined in the filtering rule and $b$ is a weight assigned during filtering. This weight is derived from the total backscattered power $I=\operatorname{tr}(T)$ (Lee et al., 1999b) and evenly applied to each component of $T$. This procedure is based upon the assumption that the noise is multiplicative, with unity mean that is uncorrelated with the image.

\subsubsection{Iterative refinement}

The filtering procedure described in the preceding section is initialized using a $3 \times 3$ window for single look datasets. If the input PolSAR coherency matrix is a rank 3 matrix we can decompose and classify without further averaging. The success of filtering depends upon accurately determining and interpreting the scattering mechanism and an accurate classification of the scattering type. As shown in Lee et al. (2008), estimates of both $H$ and $\alpha$ are close to their true value if the number of looks equals 81 or above. Unfortunately, point scatterers are smoothed when initializing with such a large window size. To correct for this, we initialize with the $3 \times 3$ window average to obtain the approximate $H / \alpha$ values and the initial fuzzy $H / \alpha$ classification of the scattering types and gradually increase the filter window size to preserve point scatterers while improving the scattering mechanism estimate and classification output. This classification output is used to adaptively filter the single look $T$. Next, we use the adaptively filtered covariance matrix to derive new $H / \alpha$ values and a new Wishart distance classification output. We then use the Wishart classification output to adaptively filter the single look $T$ using a $5 \times 5$ window. This procedure is repeated until the desired 


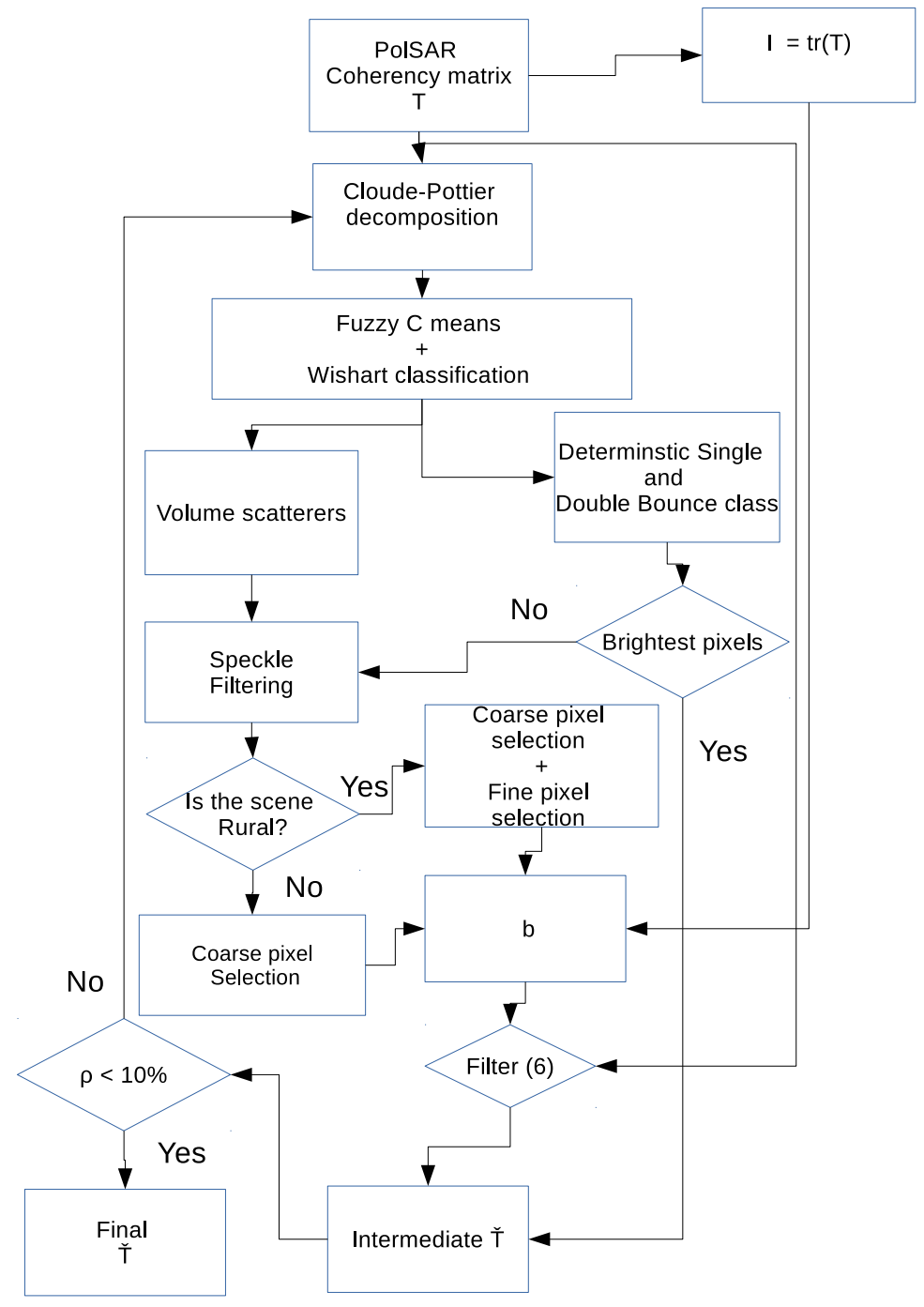

Figure 4.2: Methodology flowchart for the proposed method.

window is reached and the number of pixels that switch scattering classes is below a threshold $\rho$. In this study a $\rho<10 \%$ is used. For most data it takes 4-6 iterations for the filtering process to terminate. We used an $11 \times 11$ final window to have sufficient pixels for effective filtering. The methodology flowchart for the proposed method is given in Figure 4.2.

\subsection{PolSAR data simulation}

Speckle is present in all SAR data. Accurate quantitative evaluation of speckle filtering is only possible if a noise free image is available, which is 
the case for simulated images. Hence, to apply quantitative evaluation of the proposed speckle reduction method we adapted the Monte-Carlo PolInSAR simulation routine proposed by Cloude (2009) to PolSAR data. In addition, we reproduced the complex image structure in real PolSAR data by simulating a random image morphology.

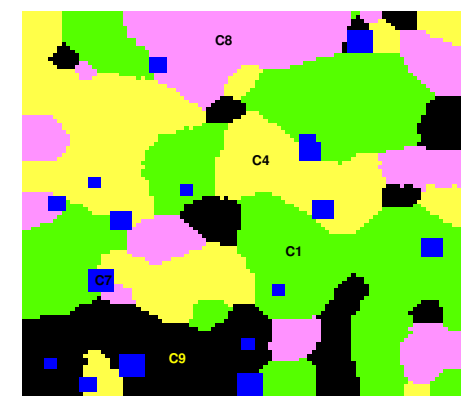

(a)

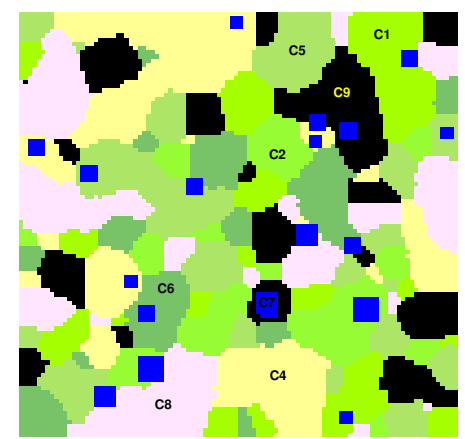

(c)

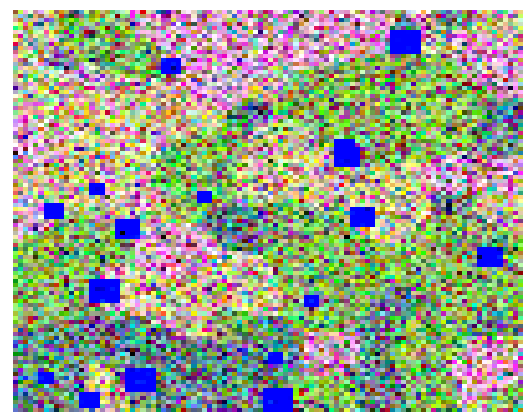

(b)

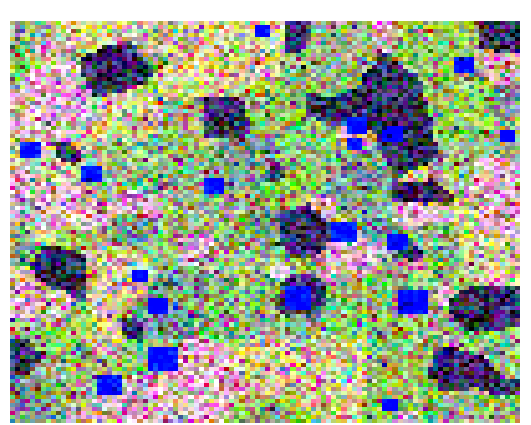

(d)

Figure 4.3: Pauli images of simulated PolSAR data. (a) Reference image derived from five scattering classes. The corresponding scattering classes are labeled as defined in Table 4.2. (b) Simulated PolSAR data derived for five scattering classes. In the images red color represents $\left|S_{H H}-S_{V V}\right|^{2}$, blue color represents $\left|S_{H H}+S_{V V}\right|^{2}$ and green color represents $\left|2 S_{H V}\right|^{2}$. (c) Reference image derived from eight scattering classes. The corresponding scattering classes are labeled as in Table 4.2. (d) Simulated PolSAR data with the same color as in (b).

Following (4.3) we assumed that the stochastic nature of the scattering vector $k$ is completely determined by the covariance matrix $C$. We first defined a coherency matrix to be used as a reference. Since the coherency matrix is a Hermitian positive semi-definite matrix, we factorized the reference coherency 
matrix $\tilde{T}$ to its respective eigenvalues and eigenvectors.

$\tilde{T}=U \Sigma U^{\dagger}$

Here, $U$ contains the eigenvectors arranged in columns and $\Sigma$ is a diagonal matrix containing the corresponding eigenvalues $\lambda$. The eigenvalues and eigenvectors generate a sequence of $n$ dimensional complex vectors $k$, with coherency matrix $T$. We next generated two independent real random sequences following Gaussian distributions, $G_{a}$ and $G_{b}$ with mean=0 and variance $=0.5$ and combined them into a complex series after scaling it by the square root of the appropriate eigenvalue.

$e=\left\{e_{j}\right\}, \quad j=1, \ldots, n, \quad e_{j}=\sqrt{\lambda_{j}}\left\{G_{a}(0,0.5)+i G_{b}(0,0.5)\right\}$.

Next, we generated $k$ by collecting $G_{a}$ and $G_{b}$ into a vector $e$ and introducing a complex correlation by multiplying this vector with the matrix of eigenvectors, i.e $k=U \cdot e$. The vector $k$ follows a zero mean complex multivariate Gaussian pdf as in (4.3).

Finally, we generated a random image morphology with different features, by using different $\tilde{T}$ matrices for different scattering classes extracted from the four look AIRSAR data (Table 4.1). We used a Markov random field (MRF) following the Gibbs distribution (Boykov et al., 2001). The parameters used to simulate a random image morphology are shown in Table 4.2.

As stated in different speckle filtering literature, a speckle filter is supposed to remove speckle noise from distributed scatterers while preserving edges and point scatterers in a PolSAR image (Lee et al., 2009). Hence, the selection of a deterministic scatterer class and a varying number of distributed scatterers is reasonable for the evaluation of PolSAR speckle filters. Hence, two simulated images were generated by taking five scattering classes (Figure 4.3a) and eight scattering classes (Figure 4.3c). Of these classes one represents a deterministic Bragg scattering and the others represent a distributed scattering with different $\alpha$ and $\mathrm{H}$ values. Speckle was added to the distributed scattering classes using (4.8) but no speckle was added to the deterministic Bragg scattering.

\subsection{Datasets}

To test the performance of the proposed method in addition to the simulated data, real airborne SAR data from the JPL/NASA AIRSAR airborne polarimetric SAR sensor (Figure 4.6a and 4.7a) was used. The AIRSAR sensor acquires fully polarimetric data $\mathrm{C}, \mathrm{L}$ and $\mathrm{P}$ band. In this study the $\mathrm{L}$ band was selected to evaluate the proposed speckle filtering method. A 


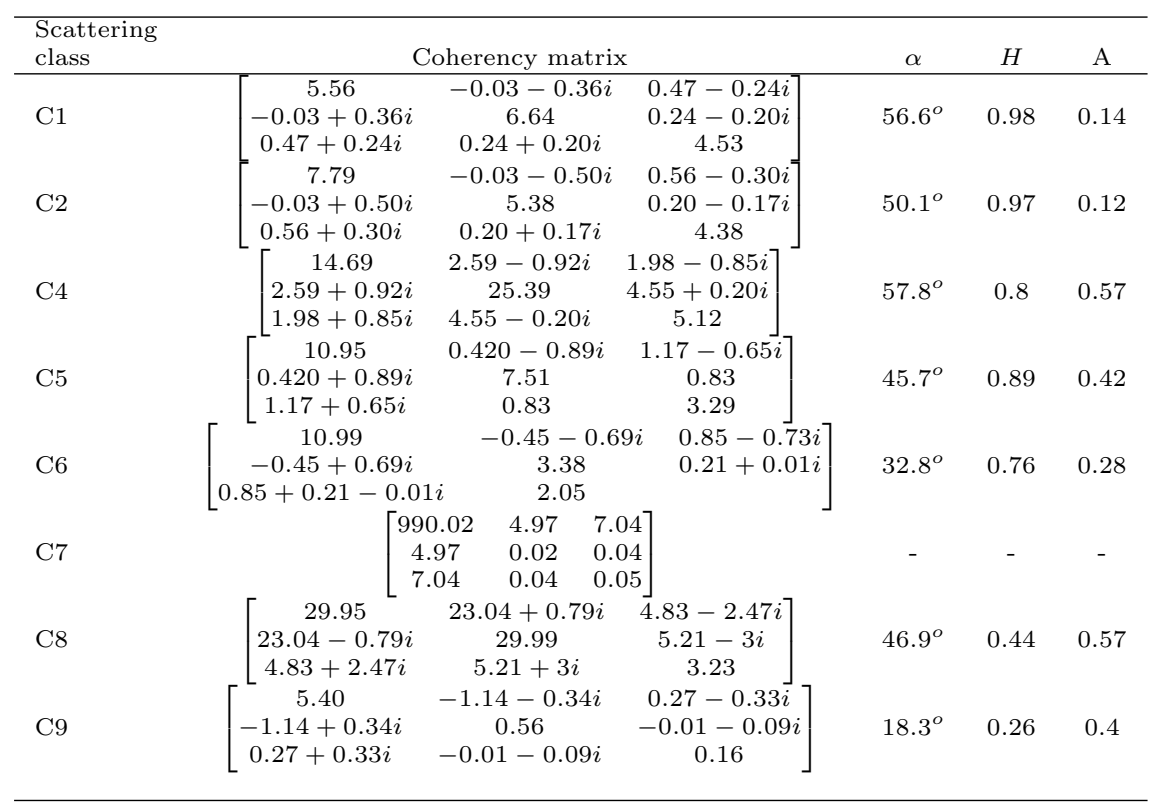

Table 4.1: Reference coherency matrix $(\tilde{T})$ for each simulated scattering class (Foucher and Lopez-Martinez, 2014). The coherency matrix in C7 is rank 1 so $\alpha, H$ and $A$ are not defined.

\begin{tabular}{lc}
\hline Parameter & Value \\
\hline Prior energy model & Potts model \\
Neighbourhood system & Second order with inverse distance weights \\
Number of iterations & 200 \\
Cooling schedule & $T_{k+1}=T_{k} \cdot \sigma$ where $T_{o}=10$ and $\sigma=0.9$ \\
Starting point & Uniform random \\
Point scatterers & randomly added \\
\hline
\end{tabular}

Table 4.2: MRF image morphology simulation parameters. 


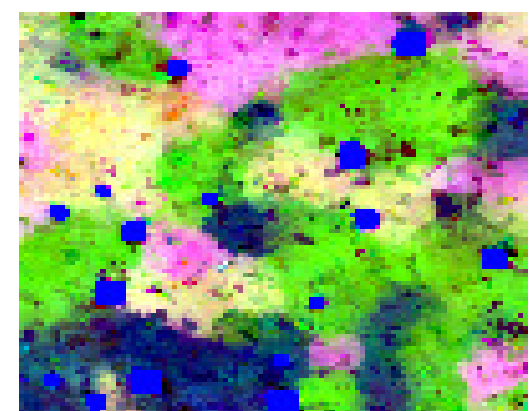

(a)

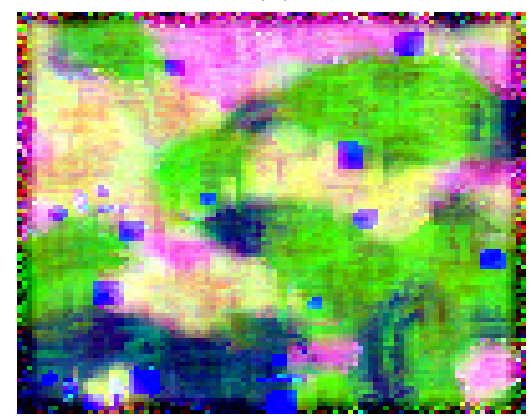

(c)

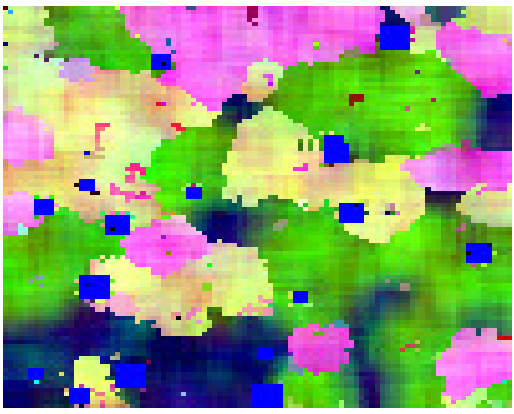

(e)

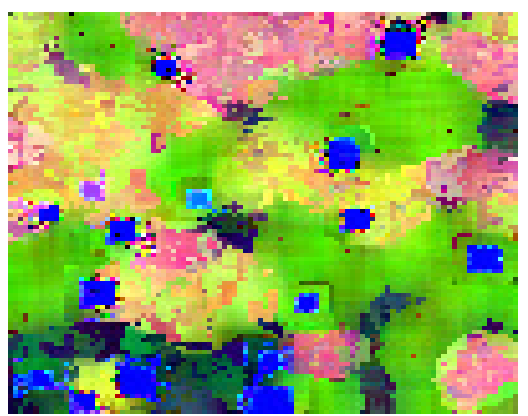

(b)

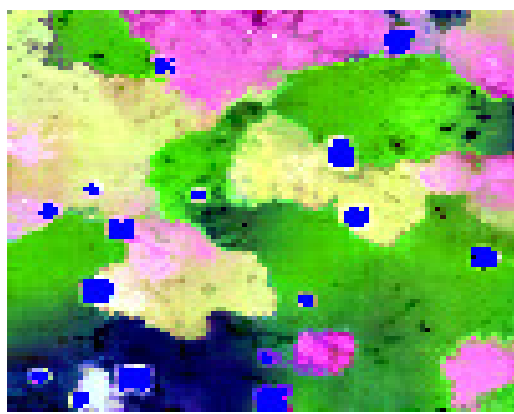

(d)

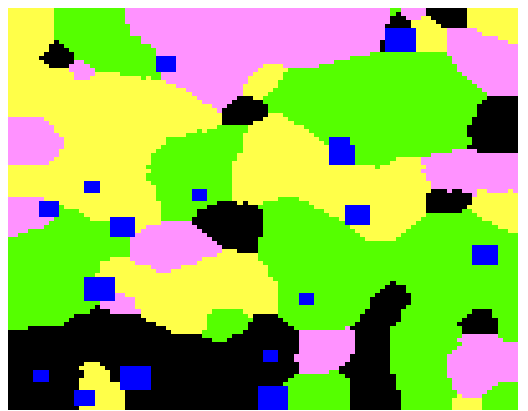

(f)

Figure 4.4: Speckle filtering results of false color composite in Pauli basis based on simulated data. The speckle free and speckled simulated data is shown in Figure 4.2. (a) Pauli channels created from IDAN filter using window size row of 80 (b) Pauli channels created from Scattering model based filter with $9 \times 9$ window. (c) Pauli channels for refined Lee filter with $9 \times 9$ window. (d) Pauli channel for Non-local means with $21 \times 21$ search window, patch size of $5 \times 5$ and threshold coefficient $=20$. (e) Pauli channel for proposed method $11 \times 11$ window 5 iteration. (f) Pauli channel for the ground truth image.

$1024 \times 900$ subset of AIRSAR data of San Francisco, USA covers mostly urban area whereas a $1024 \times 750$ subset of Flevoland, Netherlands covers almost entirely rural land. The acquisition parameters for the AIRSAR 
sensor are summarized in Table 4.3. The ground truth data from 14 feature types for the Flevoland scene was adopted from Lee et al. (2001).

\begin{tabular}{lc}
\hline Parameter & Value \\
\hline Sensor & AIRSAR airborne sensor \\
frequency/wavelength & $1.26 \mathrm{GHZ} / 23 \mathrm{~cm}$ \\
Polarization & Full \\
Range resolution & $3.75 \mathrm{~m}$ \\
Swath width (nominal) & $10 \mathrm{~km}$ \\
Off-Nadir angle (normal) & $20-60^{0}$ \\
\hline
\end{tabular}

Table 4.3: JPL/NASA AIRSAR airborne polarimetric SAR sensor acquisition parameters.

\subsection{Results}

\subsubsection{Simulated data}

To quantitatively evaluate the performance of the different speckle filtering methods, we calculated the absolute error introduced from different speckle filtering methods. Error is defined as the deviation of an estimated parameter from its true value. The average error of $\alpha$ for simulated scattering class $(i)$ is obtained as $\operatorname{Error}(i)=\operatorname{mean}\{|\tilde{\alpha}(i)-\alpha(i)|\}$, where $\alpha(i)$ is the estimated physical scattering mechanism and $\tilde{\alpha}(i)$ is the reference scattering mechanism. Similar equations apply to the bias of $H$ and $A$, respectively. The proposed method compares favorably with the other speckle filters in preserving polarimetric information (Table 4.4 and 4.5).

\begin{tabular}{lccccc}
\hline Method & $\alpha$ & $\mathrm{H}$ & $\mathrm{A}$ & $\mathrm{ENL}$ & $\mathrm{EP}$ \\
\hline IDAN & 2.109 & 0.05 & 0.068 & 14.32 & 0.904 \\
Lee & 3.1716 & 0.072 & 0.137 & 5.861 & 0.9518 \\
SMB & 1.554 & 0.053 & 0.118 & 1.4828 & 0.6233 \\
NL & 4.373 & 0.0649 & 0.103 & 7.4298 & 0.9827 \\
New & 1.6817 & 0.022 & 0.0428 & 15.014 & 0.978 \\
\hline
\end{tabular}

Table 4.4: The absolute error in $\alpha, H$ and $A$ estimates from using different speckle filtering methods and the ENL and EP calculated from different speckle filtering methods on simulated five scattering class PolSAR data. The error values for $\alpha$ are in degrees.

Next, we determined the equivalent number of looks (ENL) for each distributed scattering class, $E N L(i)=\left(\frac{\zeta(i)}{\tau(i)}\right)^{2}$, where $\zeta(i)$ is the mean and $\tau(i)$ is the standard deviation of $W$ for class $i$ and took the average of individual class ENL values. High ENL values indicate a better performance of removing 


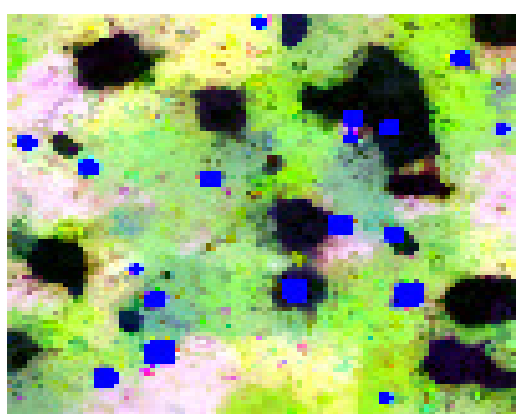

(a)

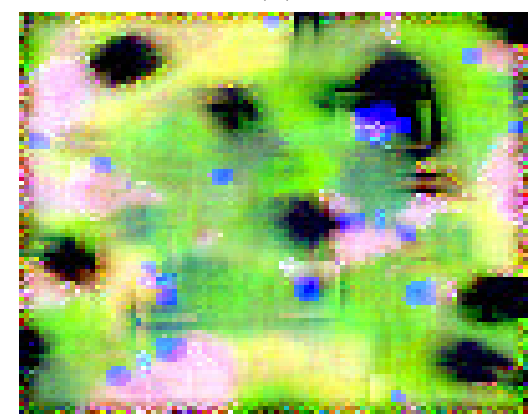

(c)

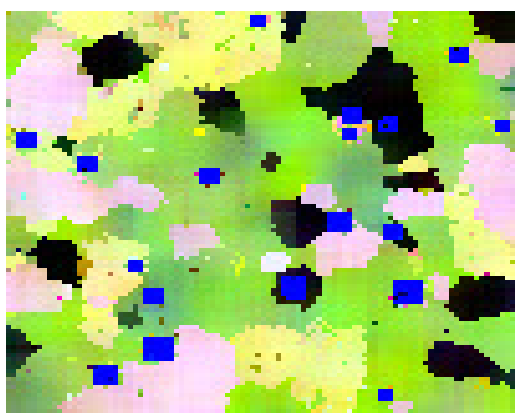

(e)

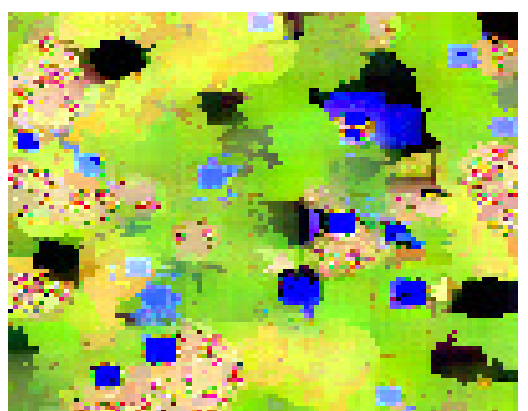

(b)

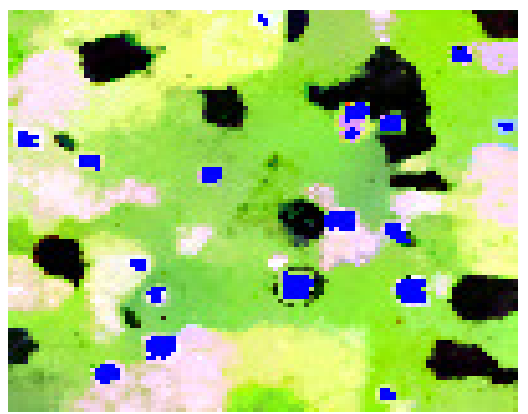

(d)

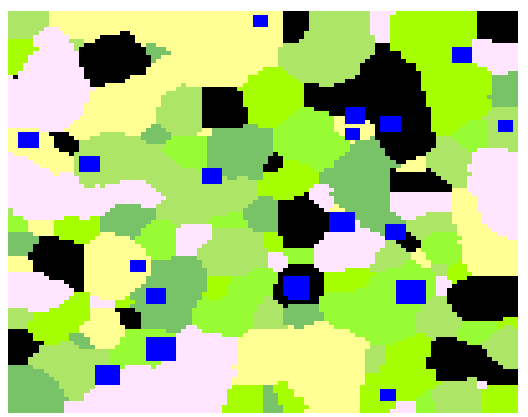

(f)

Figure 4.5: Speckle filtering results of false color composite in Pauli basis based on simulated data from 8 scattering classes. The speckle free and speckled simulated data is shown in Figure 4.2. (a) Pauli channels created from IDAN filter using window size row of 80 (b) Pauli channels created from Scattering model based filter with $9 \times 9$ window. (c) Pauli channels for refined Lee filter with $9 \times 9$ window. (d) Pauli channel for Non-local means with $21 \times 21$ search window, patch size of $5 \times 5$ and threshold coefficient $=$ 20. (e) Pauli channel for proposed method $11 \times 11$ window 5 iteration. (f) Pauli channel for ground truth image.

speckle from homogeneous regions. The proposed method compares favorably with all other evaluated methods with respect to the described 5 accuracy measures in reducing speckle from homogenous regions. 


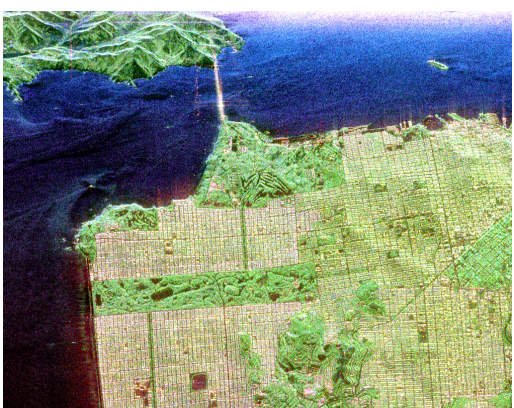

(a)

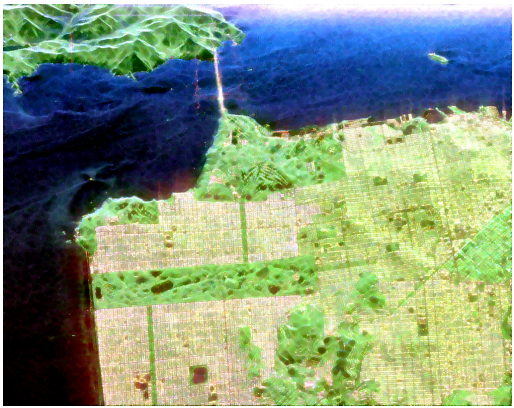

(c)

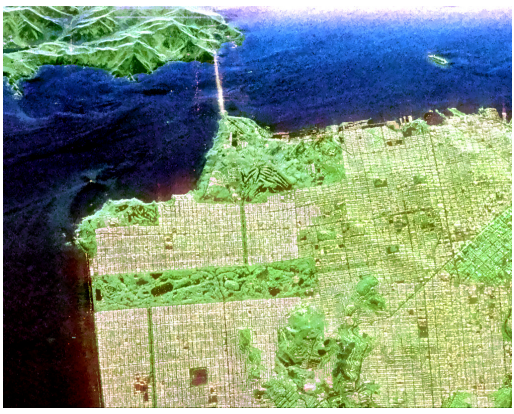

(e)

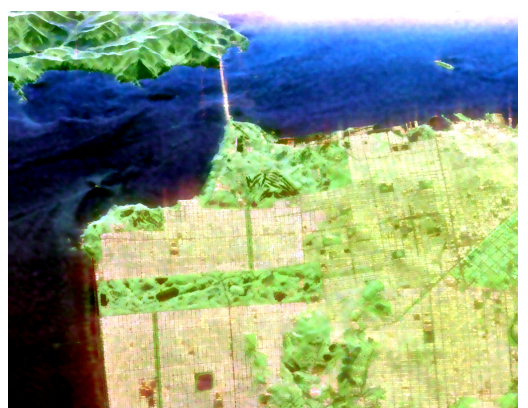

(b)

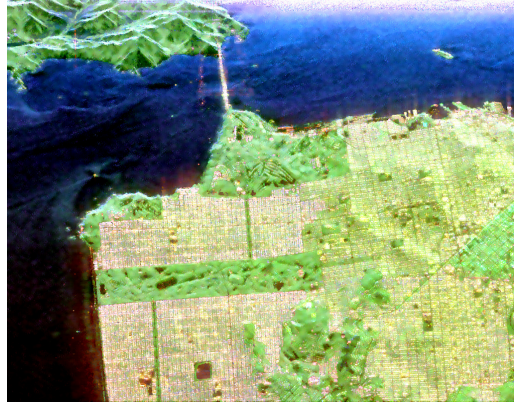

(d)

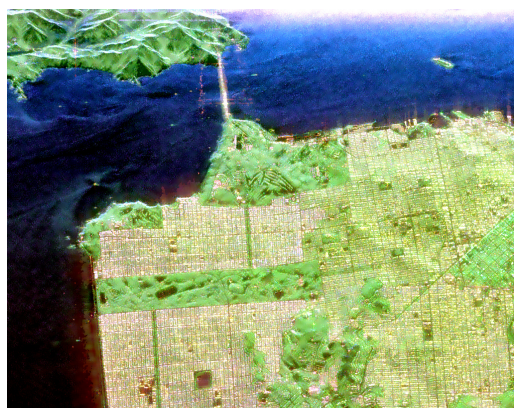

(f)

Figure 4.6: Speckle filtering results of false color composite in Pauli basis based on AIRSAR airborne L band data acquired over San Francisco. (a) Original 4 look SAR data (b) 80 window row IDAN filter. (c) Refined Lee filter with $9 \times 9$ window. (d) Scattering model based filter with $9 \times 9$ window. (e) Non-local means with $21 \times 21$ search window, patch size of $5 \times 5$ and threshold coefficient $=20$. (f) Proposed method $11 \times 11$ window 5 iteration.

To evaluate the edge preservation ability of the proposed method we implemented the edge preservation index $(E P)$ as detailed in Foucher and Lopez-Martinez (2014). We first defined the gradient preservation index $(G P)$, derived by taking the average ratio between the observed gradient values on the filtered Span image $I$ to the gradient on the reference Span image $\tilde{I}$. 


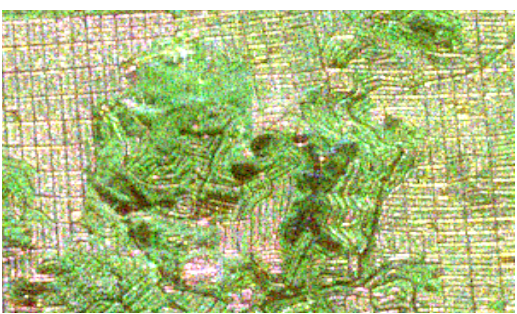

(a)

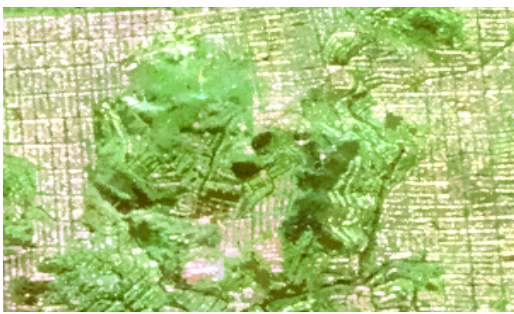

(c)

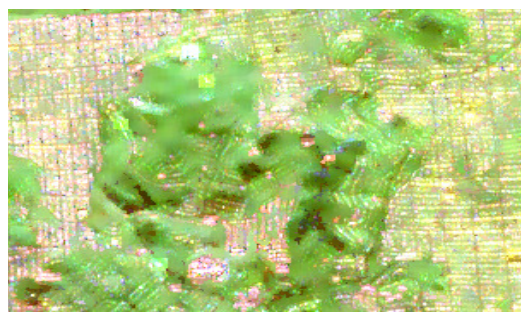

(b)

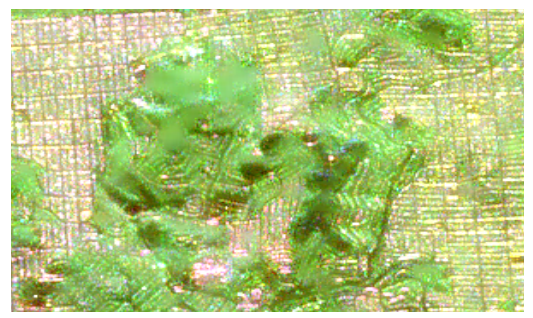

(d)

Figure 4.7: Speckle filtering of a subset of $300 \times 235$ pixels from the AIRSAR San Francisco bay image. (a) Original 4 look data. (b) Scattering model based filter with $9 \times 9$ window. (c) Non-local means with $21 \times 21$ search window, patch size of $5 \times 5$ and threshold coefficient $=20$. (d) New method $11 \times 11$ window 5 iteration.

\begin{tabular}{lccccc}
\hline Method & $\alpha$ & $\mathrm{H}$ & $\mathrm{A}$ & $\mathrm{ENL}$ & $\mathrm{EP}$ \\
\hline IDAN & 3.374 & 0.073 & 0.075 & 12.621 & 0.92 \\
Lee & 3.838 & 0.095 & 0.126 & 3.987 & 0.8463 \\
SMB & 2.353 & 0.095 & 0.1175 & 0.296 & 0.3476 \\
NL & 4.223 & 0.073 & 0.09 & 5.895 & 0.8516 \\
New & 2.208 & 0.0419 & 0.066 & 9.836 & 0.9706 \\
\hline
\end{tabular}

Table 4.5: The absolute error in $\alpha, H$ and $A$ estimates from using different speckle filtering methods and the ENL and EP calculated from different speckle filtering methods on simulated eight scattering class PolSAR data. The error values for $\alpha$ are in degrees.

$G P=\frac{1}{\nu} \sum_{l=1, . ., \nu} \frac{\sum_{L(x)=l,|\nabla \tilde{I}(x)|>0}|\nabla I(x)|}{\sum_{L(x)=l,|\nabla \tilde{I}(x)|>0}|\nabla \tilde{I}(x)|}$

Here, $\nabla$ is the Sobel gradient operator, $L(x)$ is the class label for pixel $x$ and $\nu$ is the number of distributed scattering classes. $E P$ is derived by projecting 


\begin{tabular}{lc}
\hline Method & Computation time, s \\
\hline IDAN & $3.97 \times 10^{-5}$ \\
Refined Lee & $4.02 \times 10^{-5}$ \\
SMB & $1.99 \times 10^{-4}$ \\
NL & $2.72 \times 10^{-4}$ \\
Proposed method (coarse selection, 5 iterations) & $2.1 \times 10^{-3}$ \\
Proposed method (fine selection, 5 iterations) & $6 \times 10^{-3}$ \\
Proposed method (coarse (2 iterations) and & \\
fine selection (3 iterations) & $4.4 \times 10^{-3}$ \\
\hline
\end{tabular}

Table 4.6: Speckle filtering methods and their respective computational efficiency measured per pixel when run using an Intel Core i7 CPU with 8GB memory run on a 64 bit Linux environment.

the values of $G P$ in the interval $[0,1]$ using the triangular function given as:

$E P= \begin{cases}1-|1-G P|, & G P<2 . \\ 0, & G P \geq 2 .\end{cases}$

We took the average for each scattering class to evaluate the overall edge preserving performance. Low values of $E P$ indicate edge over-filtering or under filtering, whereas a high $E P$ value indicates a good edge preservation. We observe that the proposed method performs well in preserving edges in both simulated data.

To investigate the efficiency of the proposed filter an image profile was created to compare $\left|S_{H H}+S_{V V}\right|^{2}$ intensity profile with the other state of the art filtering methods (Figure 4.9). The new filter achieves the best filtering results as compared with the true $\left|S_{H H}+S_{V V}\right|^{2}$ intensity. The non-local means and the proposed method adequately remove speckle while maintaining deterministic point scatterers, whereas the poor edge preservation performance of SMB is clearly indicated in Figure 4.9a.

\subsubsection{Airborne AIRSAR data}

Figures 4.6 and 4.8 show that the IDAN filter performs well in preserving edges but it did not fully remove the speckle noise found around volume scatterers. SMB was not successful in preserving point scatterers and edges. Furthermore, it introduced artifacts throughout the image (Figure 4.8c). The refined Lee filter preserved strong point scatterers but smoothened out other point scatterers. The non-local means had a variety of results when used in conjunction with the refined Lee and Lee sigma filter (Lee et al., 2009). It preserved point scatterers but a general over-filtering is observed when used with refined Lee filter. It achieved its best result when used with the Lee sigma filter with a $21 \times 21$ search window, $3 \times 3$ target search window, patch 
size of $5 \times 5$, a $9 \times 9$ general filter window and a threshold coefficient equal to 20. The proposed method, was successful in preserving point scatterers and edges while reducing speckle noise using an $11 \times 11$ window and 5 iterations.

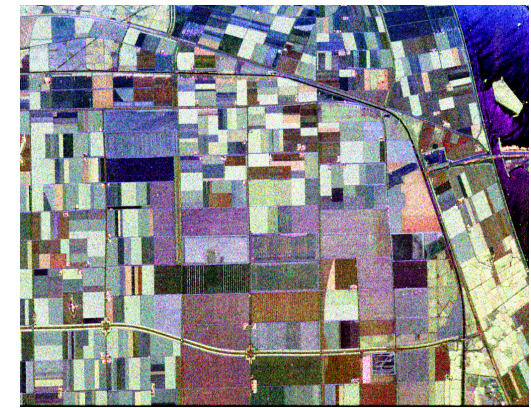

(a)

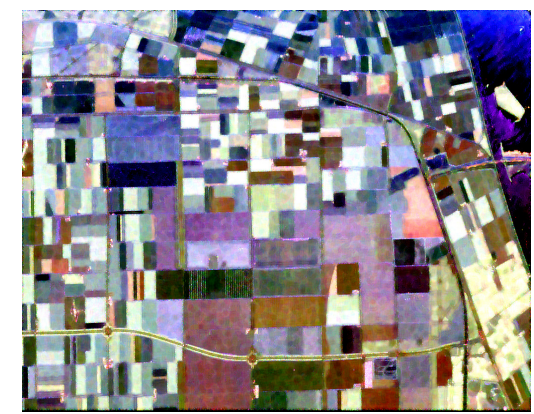

(c)

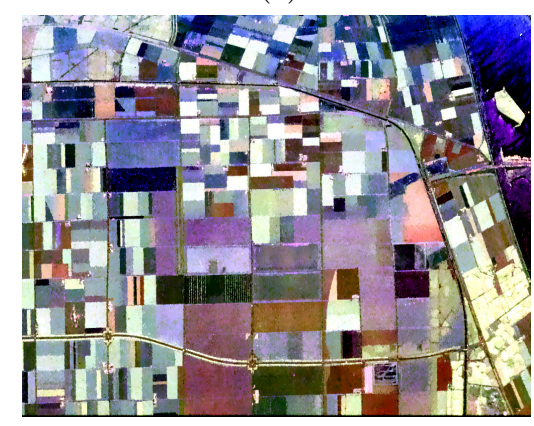

(e)

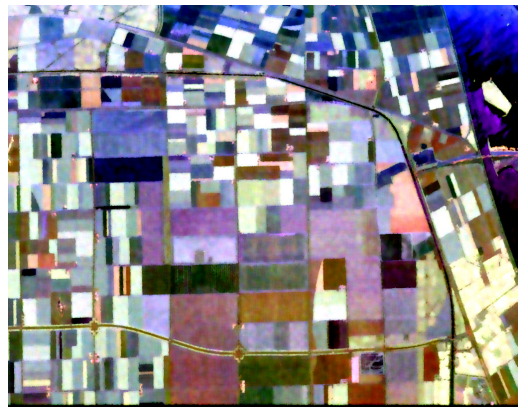

(b)

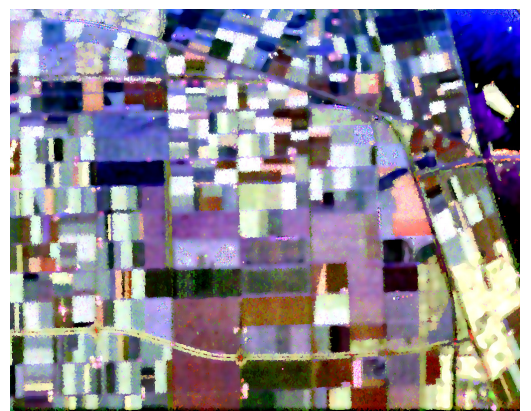

(d)

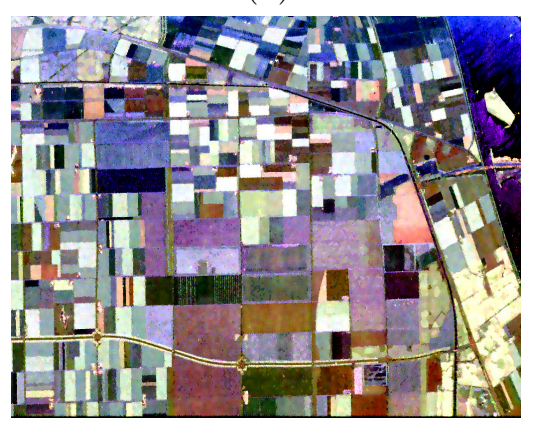

(f)

Figure 4.8: Speckle filtering results of false color composite in Pauli basis based on AIRSAR airborne L band data acquired over Flevoland, Netherlands. (a) Original 4 look SAR data. (b) 80 window row IDAN filter. (c) Refined lee filter with $9 \times 9$ window. (d) Scattering model based filter with $9 \times 9$ window. (e) Non-local means with $21 \times 21$ search window, patch size of $5 \times 5$ and threshold coefficient $=20(\mathrm{f})$ Proposed method $11 \times 11$ window 5 iteration .

For AIRSAR data two separate experiments were conducted in a completely rural scene over Flevoland, and a mixture of urban and natural over San 
Francisco bay region. The refined Lee and scattering model based filters blurred the edges between different agricultural land parcels and roads (Figure 4.8). The non-local means filter and the new method both preserved edges while reducing speckle. The non-local means and the new method performed well in reducing speckle while preserving the boundaries between different agricultural land parcels while slight over-filtering was observed on built-up found in the farm lands. In this regard, the new method performed well in preserving the isolated built up features in the image. In the San Francisco bay scene (Figure 4.7), the scattering model based filter produced better preservation of points and edges. However, subtle image features were still over filtered. The non-local means filter performed well in preserving edges but it also over filtered built up features that are mixed with vegetation whereas the proposed filter preserved these mixed features.

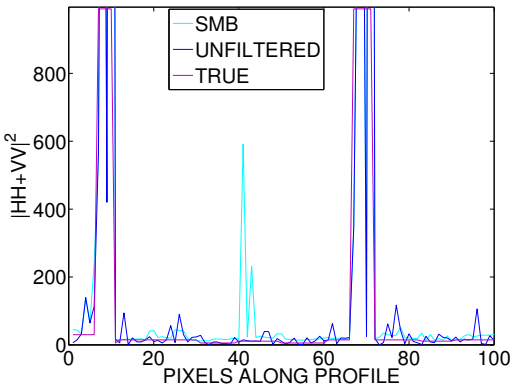

(a)

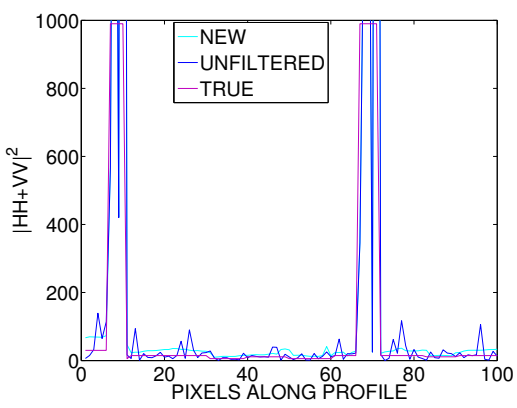

(c)

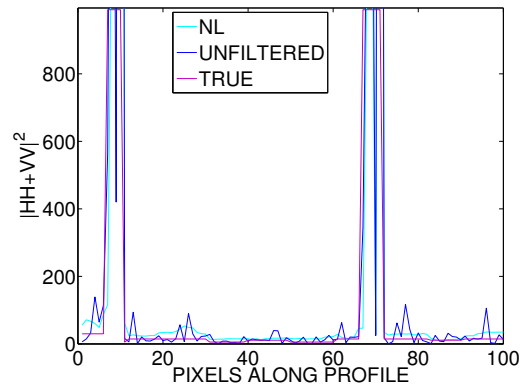

(b)

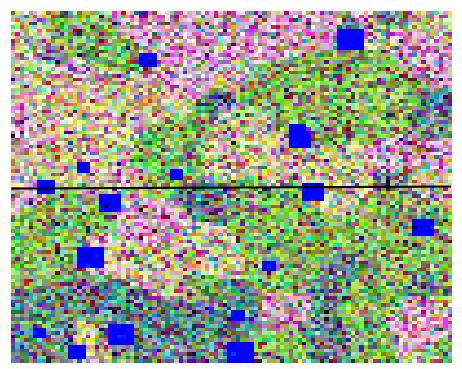

(d)

Figure 4.9: Intensity profile from simulated data for $\left|S_{H H}+S_{V V}\right|^{2}$ for the different speckle filters as compared with the original unfiltered data and the ground truth image. (a) Scattering model based filter. (b) Non-local means filter (c) proposed method (d) Location where the profile was taken.

The proposed method compared favorably with other state of the art speckle filters in preserving point scatterers and edges, even though a block effect is observed around edges because the filter was initialized using a $3 \times 3$ boxcar average. The non-local means filter (NL) also performed well in preserving points but it showed some deficiency in maintaining edges near feature bound- 
aries. The refined Lee filter failed to preserve many point scatterers and resulted in over-filtering in all regions. The scattering model based filter (SMB) blurred all point scatterers and edges and the IDAN filter preserved the points but failed to filter out the speckle noise from homogeneous regions (Figure 4.6 and 4.8).

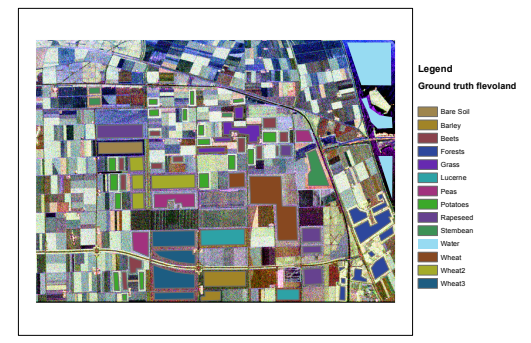

(a)

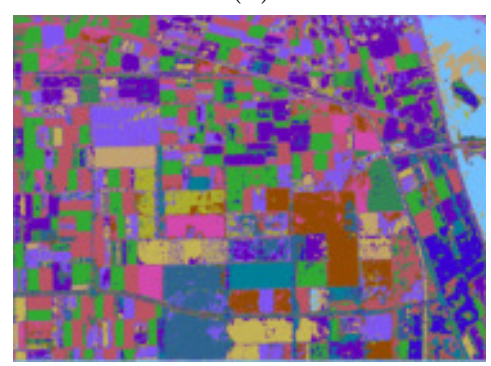

(c)

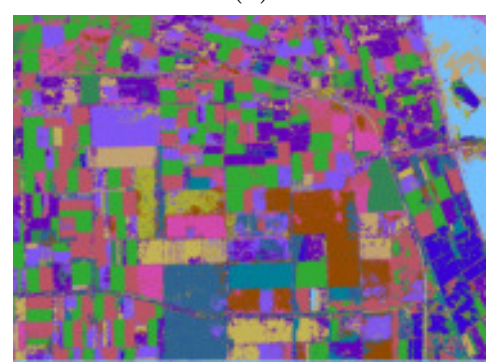

(e)

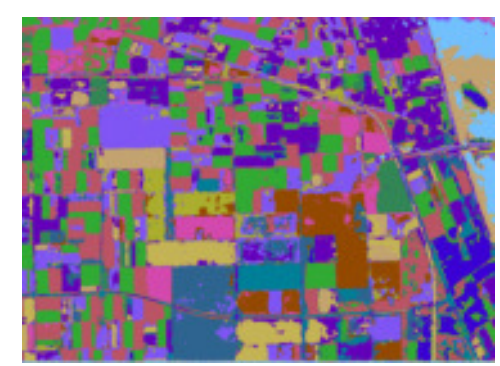

(b)

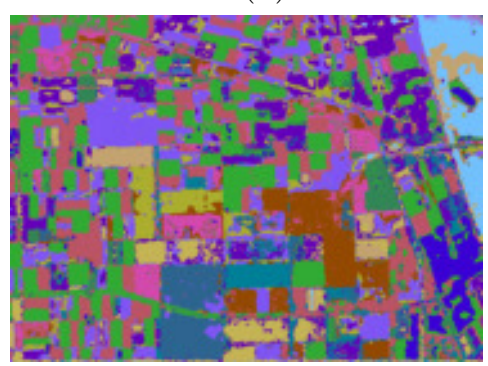

(d)

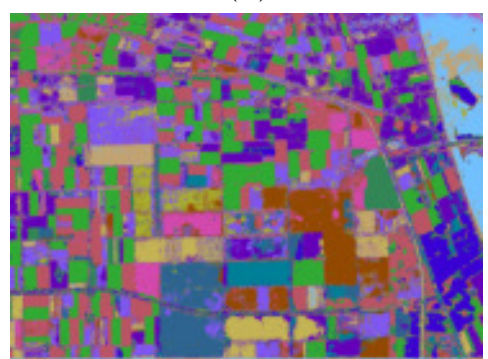

(f)

Figure 4.10: Supervised Wishart classification of AIRSAR airborne L band data acquired over Flevoland, Netherlands. (a) Ground truth data adopted from (Lee et al., 2001) (b) IDAN filter (c) Refined Lee filter (d) SMB (e) NL (f) Proposed method.

\subsubsection{Effect of speckle filtering for thematic applications}

To evaluate the performance of the proposed speckle filter we applied thematic classification to speckle filtered PolSAR data from different speckle filters 
and compared the classified output with ground truth data adopted from Lee et al. (2001). Before applying classification we split the ground truth data into training area and a test area. A supervised Maximum Likelihood Wishart distance classifier (Lee et al., 1994a) was implemented on the IDAN, refined Lee, SMB, NL and the proposed method were applied.

\begin{tabular}{lcc}
\hline Method & observed accuracy & kappa coefficient \\
\hline IDAN & 0.9161 & 0.9084 \\
Refined Lee & 0.9181 & 0.9105 \\
SMB & 0.9132 & 0.9051 \\
NL & 0.9045 & 0.8954 \\
Proposed Method & 0.9074 & 0.8987 \\
\hline
\end{tabular}

Table 4.7: The classification accuracy for the speckle filtered PolSAR data using different methods.

It can be observed from Table 4.7 that the classification accuracy of the proposed method is slightly lower than that of IDAN, refined Lee and SMB. This is due to the generalized feature type indicated by the ground truth (Figure 4.10), which differs slightly from the signal variation from the PolSAR data. NL and the proposed method were both designed to preserve spatial details in the PolSAR image. This results in deviation from the generalized ground truth image. To improve the classification accuracy for the proposed method it is recommended to use a larger than $11 \times 11$ window.

To analyze the effect of speckle filtering on urban scatterers we analyzed the canonical scattering mechanisms from the AIRSAR San Francisco scene. We selected a $100 \times 100$ patch from the image scene that was built up. We applied Cloude-Pottier eigenvalue and eigenvector decomposition and the unsupervised Wishart classification to derive the scattering mechanisms from speckle filtered PolSAR data. A ratio analysis of the scattering contribution from the three canonical scattering mechanisms after applying different speckle filters was derived.

\begin{tabular}{lccc}
\hline Method & Single Bounce & Double bounce & Volume \\
\hline Refined Lee & $0.5 \%$ & $9.91 \%$ & $89.58 \%$ \\
SMB & $1.7 \%$ & $11.36 \%$ & $86.93 \%$ \\
NL & $0.009 \%$ & $9.27 \%$ & $90.71 \%$ \\
Proposed method & $1.54 \%$ & $12.16 \%$ & $86.28 \%$ \\
4 look original data & $2.46 \%$ & $18.34 \%$ & $79.19 \%$ \\
\hline
\end{tabular}

Table 4.8: Scattering mechanism contribution after application of different speckle filtering methods.

It can be observed from Table 4.8 that the proposed method preserves the 
double bounce component better than the other speckle filtering methods. Different thematic application require different levels of filtering. As indicated in Table 4.7 thematic applications involving agriculture and forestry require more filtering hence preservation of subtle variations may not be important. With the exception of precision agriculture. On the contrary, urban applications such as characterization of urban scattering often requires preservation of resolution. Hence the optimal speckle filter should perform well in both applications. In this regard, the proposed speckle filter achieved good result. A future study should further analyze the effect of speckle filtering on different thematic applications.

\subsection{Discussion}

The proposed method works well with $\mathrm{L}$ band PolSAR data. It should be further evaluated for application with short wavelength SAR data in particular for X band high resolution airborne PolSAR data. For instance, the rule deriving the assignment of scattering classes for filtering should be adjusted when using short wavelength high resolution sensors because pixels defined as surface scatterers in L band will be volume scatterers in X band.

We selected the Cloude-Pottier eigenvalue-eigenvector decomposition to replace the Freeman-Durden model based decomposition to minimize the a-priori information requirement in the image scene and minimize the bias from the decomposition method. The Cloude-Pottier eigenvalue-eigenvector decomposition is also roll invariant. Hence, an extra processing step of orientation angle compensation is not required. To minimize the pixel selection bias we applied the fine pixel selection in volume scatterers in which all pixels with different scattering properties are not filtered with each other. In addition, MMSE is applied to all pixels in each scattering category which resulted in better preservation of edges in volume scatterers. To mitigate the bias introduced by using a small number of samples in estimating the scattering mechanisms and classification we adopted an iterative decomposition and classification approach. Initializing with a fuzzy $H / \alpha$ classification helped to achieve higher accuracy than crisp Wishart $H / \alpha$ classification. Since speckle level is minimized we applied the crisp Wishart distance classification on subsequent iterations thus maintaining a high classification accuracy while improving computational efficiency. The combination of these three steps resulted in an overall improvement of the speckle reduction results (Figure 4.4-4.8).

The initial fuzzy $H / \alpha$ classification to designate the different scattering mechanisms resulted into improved classification which is critical for a successful application of this method. Fuzzification of the $H / \alpha$ plane led to improved identification and consequent classification of scattering mechanisms. In addition, coarse pixel selection was effective in filtering scenes with a mixture of urban and rural features as in the San Francisco scene. For dominantly 
rural scenes as in the simulated data and the Flevoland scene, best results were obtained for a mixture of coarse and fine pixel selection. Initial classification of scattering mechanisms was also attempted using the crisp unsupervised Wishart distance classifier (Lee et al., 1999a) and compared with the fuzzy $H / \alpha$ classification. Both classifiers correctly classified surface scatterers (Bragg and random surface scattering) but the unsupervised Wishart classifier underestimated the dihedral scattering component identified on the $H / \alpha$ plane.

The efficiency of the speckle filters for different thematic applications is different depending on the design parameters of the filter. If the filter is designed to preserve edges it tends to over filter the homogeneous regions in the image thereby more suited for agricultural application. The proposed method was designed to have enough trade off in between smoothing features, preserving edges and point features. Hence, it under performs as compared with IDAN, refined Lee and SMB in thematic applications where spatial variation is un-important as in small scale agricultural applications. This can be mitigated by using a larger than $11 \times 11$ filter window. In urban thematic applications the proposed method compares favorably with other speckle filters because the filter is designed to preserve polarimetric information, spatial details and point scatterers.

The proposed method has a limitation of achieving good result at the price of computational time (Table 4.6). This can be prohibitive when processing a large image scene consisting of millions of pixels. This is the main drawback observed from employing the new filtering approach. To reduce the computational time the fuzzy $H / \alpha$ classification can be replaced with the crisp unsupervised Wishart classification at the risk of less accurate scattering type classification output. The proposed technique can be easily extended to filter dual polarization data obtained from sensors such as Sentinel-1. It can also be extended to filter PolInSAR data for coherence estimation. In the current study the scope was limited to qualitative and quantitative evaluation of speckle filtering performance. In future work comparisons should be made with ground truth data to verify the efficacy of the proposed speckle filtering method.

\subsection{Conclusions}

The proposed method expands the scattering model based polarimetric speckle filter (Lee et al., 2006). The study shows that the proposed method compares favorably to other methods such as the refined Lee filter, the scattering model based filter and the non-local means filter. We conclude that it performs robustly in a variety of image scenes. However, for small scale agricultural applications it does not perform well. We further conclude that the strength of the proposed method lies in accurately classifying scattering mechanisms that are obtained by iteratively refining the $H / \alpha$ and coherence 
matrix estimates. Its performance compares favorably with the edge aligned filtering of the refined lee or the patch based Wishart similarity test method implemented in the non-local means filter. The main observed limitation is that it is computationally expensive. 


\section{Polarimetric scattering property based distributed scatterer processing method for PSI applications}

\section{Chapter summary}

This chapter extends the method that resulted in the spatial adaptive filter based on the dominant scattering mechanism. It is tuned to a multi-temporal PolInSAR data stack. The extended method is based on distinguishing permanent scatterer (PS) and distributed scatterers (DS) candidates. This is based on phase entropy. It selects coherent scattering mechanisms within a DS candidate and discards any incoherent scattering mechanism. The method increases the number of pixels available for deformation measurement. The reliability of the new method is tested on both dual polarized and fully polarized SAR data and compared to other state of the art multi-temporal InSAR methods.

This chapter is based on: Mullissa, A. G., Perissin, D., Tolpekin, V., Stein, A., Polarimetric scattering property based distributed scatterer processing method for PSI applications. IEEE Transactions in Geoscience and Remote Sensing (Minor revision) 


\begin{abstract}
Permanent scatterer interferometry (PSI) is a multi-temporal interferometric synthetic aperture radar (InSAR) technique that derives high accuracy ground deformation measurement. A high density of persistent scatterers (PS) is desired to improve the accuracy of phase unwrapping in deformation measurement. In natural environments with low PS density, distributed scatterers (DS) could serve as additional radar targets. To improve the signal to noise ratio of DS and preserve PS used in PSI analysis, spatially adaptive filtering is required. This chapter introduces a polarimetric scattering mechanism based adaptive filtering method that preserves PS candidates and filters DS candidates. To further improve the coherence estimate of DS candidates the technique includes a complex coherence decomposition that allowed us to adaptively select the coherent scattering mechanisms, thus improving pixel coherence estimate. The proposed method was evaluated on 11 quad polarized ALOS PALSAR images and 21 dual polarized Sentinel-1 images acquired over San Fernando valley, California and Groningen, The Netherlands, respectively. The application of this method increased the number of coherent pixels by almost a factor of 8 as compared with a single polarization channel. The study concludes that coherence estimate can be significantly improved by applying scattering property based adaptive filtering and coherence matrix decomposition and that accurate displacement measurements can be achieved.
\end{abstract}

Keywords: Adaptive filtering, Polarimetric Optimization, Multi-temporal InSAR, Polarimetric SAR Interferometry (PolInSAR), Distributed scatterers. 


\subsection{Introduction}

Multi-temporal InSAR is a well studied Earth observation technique that provides a mm scale accuracy in ground deformation measurements (Ferretti et al., 2001), (Hooper, 2008), (Berardino et al., 2002), (Crosetto et al., 2008), and (Perissin and Wang, 2012). It aims to identify stable radar targets which are strong deterministic scatterers and exploits their coherent nature to measure ground displacement. To derive accurate model fitting for displacement measurement a dense network of permanent scatterer (PS) candidates is required increase the accuracy of phase unwrapping. Hence, multi-temporal InSAR works well in urban areas where the scattering from buildings and other man made structures guarantee a dense network. However, it has achieved limited success in obtaining dense PS candidates in natural environments.

To overcome this limitation, extended radar targets termed distributed scatterers (DS) were exploited to obtain dense pixel candidates. These targets provide moderate coherence as they are affected by temporal and baseline decorrelation. Hence, by applying adaptive spatial filtering techniques the signal to noise ratio (SNR) for these targets may be improved. In previous works different statistical methods were used to distinguish PS and DS candidates. Goel and Adam (2014) applied the Anderson-Darling test statistic and Ferretti et al. (2011) used the Kolmogorov-Smirnov test to evaluate the similarity of the amplitude distribution in two image pixels. Lin and Perissin (2017) applied a robust $t$-test to improve the effectiveness of identifying statistical homogeneous pixels (SHP). With the availability of fully polarimetric data, Navarro-Sanchez and Lopez-Sanchez (2014) applied a likelihood ratio test to establish the similarity of two Wishart matrices in two pixels. To compute the interferometric coherence, pixels are categorized into SHP on which spatial averaging is performed. Perissin and Wang (2012) selected DS candidates by appling weights to interferograms to identify DS that are coherent in portions of the interferogram stack. This method relaxed restrictions imposed by the PSI technique proposed by Ferretti et al. (2001).

When using medium resolution SAR data, both in mixed rural-urban areas and rural areas, different DS scattering mechanisms are mixed. An example is a mixed pixel containing buildings, car and trees. Even though the SNR quality is improved by multilooking, the interference between the mixed scattering mechanisms decreases the overall coherence estimate in the resolution cell. This has important implications in deformation measurements when some of these scattering mechanisms are coherent and some are not. Fornaro et al. (2015) introduced the Component extrAction and sElection SAR (CAESAR) method that is based on using a principal component analysis to decompose the interferometric covariance matrix to mitigate the effects of layover in urban areas. This method was able to reduce the effects of decorrelation in the DS pixels. Recently, Cao et al. (2016) applied eigen decomposition on the interferometric coherence matrix to obtain the dominant scattering mechanism within the DS pixel. This method is effective in reducing decorrelation 
by selecting the dominant scattering mechanism to derive the interferometric phases. It assumes however that all secondary scattering mechanisms may constitute noise.

In this chapter, a polarimetric scattering mechanism based adaptive spatial filtering is introduced to improve SNR of DS candidates. The interferometric coherence matrix decomposition method (Cao et al., 2016) is modified to adaptively select secondary scattering mechanisms that interfere constructively with the dominant scattering mechanism and later applied in Quasi permanent scatterer (QPS) interferometry (Perissin and Wang, 2012). The objective of this chapter is to adaptively filter DS candidates and select the most stable scattering mechanisms in the DS cell to improve the overall pixel coherence in a mixed urban-rural scene.

This chapter is organized as follows. The methodology employed in this study is described in Section 5.2. The datasets used in this study in Section 5.3. Results obtained from the methods are shown in Section 5.4. Discussions and conclusions on the obtained results are presented in Section 5.5.

\subsection{Method}

\subsubsection{SAR polarimetry}

Fully polarimetric SAR sensors measure the scattering matrix $S$ which can be presented as:

$S=\left[\begin{array}{ll}S_{H H} & S_{H V} \\ S_{V H} & S_{V V}\end{array}\right]$,

where the complex scattering coefficient $S_{X Y}$ indexed as $X, Y=(H, V)$ represents the horizontal $(H)$ and vertical $(V)$ polarization states. In SAR polarimetry, this scattering matrix is represented by the target scattering vector $\tilde{k}$. Vectorization expands the scattering matrix using simpler canonical scattering mechanisms. The Pauli target scattering vector of a given scattering matrix in the monostatic case assuming reciprocity i.e $S_{H V}=S_{V H}$, is given as (Lee and Pottier, 2009)

$\tilde{k}=\frac{1}{\sqrt{2}}\left[\begin{array}{lll}S_{H H}+S_{V V} & S_{H H}-S_{V V} & 2 S_{V H}\end{array}\right]^{T}$,

where ${ }^{T}$ designates a matrix transpose. In dual polarimetric data as in Sentinel-1 configuration (VH VV), $\tilde{k}$ reduces to:

$\tilde{k}=\left[\begin{array}{ll}S_{V V} & 2 S_{V H}\end{array}\right]^{T}$.

For deterministic point scatterers $\tilde{k}$ describes the scattering process completely. For distributed scatterers, however, $\tilde{k}$ displays a random property depending 
on the condition that the SAR wavelength is smaller than the resolution cell. Hence, $\tilde{k}$ is modeled by a multivariate complex circular Gaussian probability density function (Lee et al., 1994b). The second order statistics represented by the coherency matrix $T$ that completely define the randomness of $\tilde{k}$ are computed by assuming stationarity and ergodicity, given as:

$T=\left\langle\tilde{k} \tilde{k}^{\dagger}\right\rangle=\frac{1}{n} \sum_{i=1}^{n} \tilde{k} \tilde{k}^{\dagger}$

Here \langle\rangle is spatial or temporal average of pixels and $n$ is the number of samples or looks used to estimate $T$. In this chapter, stationarity and ergodicity is assumed in time so $n$ represents the number of images in the stack. Notice that no spatial averaging is applied since $n$ is obtained from the temporal dimension. $T$ is modeled by the complex Wishart probability density function (Goodman, 1963).

\subsubsection{Target decomposition}

Target decomposition theorems are used to determine the dominant scattering mechanism present in a PolSAR data. The Cloude-Pottier eigenvalueeigenvector decomposition method (Cloude and Pottier, 1997) break down $T$ into a sum of elementary scattering contributions. $T$ is a Hermitian positive semi-definite matrix, hence it can be factorized into a matrix of eigenvalues and eigenvectors. Thus,

$T=\sum_{i=1}^{3} \sigma_{i} u_{i} u_{i}^{\dagger}$

where $u_{i}$ is the eigenvector and $\sigma_{i}$ is the eigenvalue. The eigenvector can be further expanded by using physical parameters of targets i.e.

$u_{i}=\left[\begin{array}{lll}\cos \alpha_{i} & e^{j \delta_{i}} \sin \alpha_{i} \cos \beta_{i} & e^{j \psi_{q} i} \sin \alpha_{i} \sin \beta_{i}\end{array}\right]^{T}$.

Here $\alpha$ refers to the physical scattering mechanism as described in (Cloude and Pottier, 1997), $\beta$ refers to the orientation of the target within the RADAR line of sight and $\delta$ and $\psi_{q}$ are the co-polar and cross polar phase angles, respectively. The average physical scattering mechanism $\tilde{\alpha}$ is obtained as:

$\tilde{\alpha}=\sum_{i=1}^{3} P_{i} \alpha_{i}$, where $P_{i}=\frac{\sigma_{i}}{\sum_{i=1}^{3} \sigma_{i}}$. 
Here $P_{i}$ is the probability obtained from the eigenvalues $(\sigma)$. To describe the statistical disorder of the scattering mechanisms, the scattering entropy is used, given as:

$$
H=-\sum_{i=1}^{3} P_{i} \log _{3} P_{i} .
$$

In the Cloude-Pottier eigenvalue-eigenvector decomposition $\tilde{\alpha}$ typically assumes a value between $0^{0}$ and $90^{\circ}$ and $H$ a value between 0 and 1 . $\tilde{\alpha}$ values that are close to $0^{0}$ resemble a surface scattering, whereas $\tilde{\alpha}=45^{0}$ resembles a volume scatterer and $\tilde{\alpha}=90^{\circ}$ indicates a double bounce scattering mechanism. $H$ values close to 0 represent a deterministic scattering mechanism and an $H$ value close to one represents a random scattering where there is no dominant scattering mechanism. However, $\tilde{\alpha}$ by itself is not enough to define the scattering mechanisms hence, the different scattering mechanisms are described in the $H / \tilde{\alpha}$ plane (Lee et al., 1999a).

\subsubsection{Homogeneous pixels selection}

To preserve PS candidates in a multitemporal InSAR processing and filter DS candidates, homogeneous pixel patches should be identified. In this chapter, similarity between the objective pixel to be filtered and its neighboring pixels within a neighbourhood window is established based on similar scattering properties within spatially interconnected pixels.

The Cloude-Pottier eigenvalue-eigenvector decomposition (Cloude and Pottier, 1997) provides the scattering properties i.e average scattering mechanism $(\tilde{\alpha})$ and scattering entropy $(H)$ in each resolution cell. We derive the neighborhood similarity by classifying the scattering mechanisms. This is accomplished by implementing the Wishart distance measure (Lee et al., 1999a) between a pixel's $T$ estimate (5.4) and the coherency matrix of the $m^{\text {th }}$ scattering mechanism class, $V_{m}$. Here $V_{m}=E[T]$ for $T \in \omega_{m}$ and the distance measure between pixel $T$ and $V_{m}$ is given as:

$d\left(T, V_{m}\right)=\ln \left|V_{m}\right|+\operatorname{Tr}\left(V_{m}^{-1} T\right)$.

Here, $\omega_{m}$ are the pixels belonging to the $m^{\text {th }}$ scattering mechanism class. The classification is implemented using an iterative combination of the unsupervised target decomposition classifier and the supervised Wishart classifier (Lee et al., 1999a). The initial scattering mechanism class was derived for each pixel by taking the zone center in the $H / \tilde{\alpha}$ scatter plot (Cloude and Pottier, 1997). For dual polarized data the $H / \tilde{\alpha}$ zone is modified as discussed in (Ji and $\mathrm{Wu}, 2015)$. In subsequent classifications the average $T$ from each scattering class defined in the $H / \tilde{\alpha}$ plane is used to define the new class coherence matrix $V_{m}$ and each pixel in the image is classified based on the new $V_{m}$. This process is repeated until a termination criterion is reached. 
In this chapter, the number of pixels switching class when compared with a previous classification result is used.

The SHP's are defined within a $p \times p$ pixel window. We first discard isolated pixels that do not belong to a scattering class of the objective pixel to be filtered that is not spatially connected directly or through other SHP's. The spatially adaptive filtering is defined for pixel $W$ and all SHP's connected to pixel $W$. The brightest pixels that belong to the deterministic single bounce or double bounce class are left out as these may be PS candidates. This is accomplished by using the concept utilized in the Lee sigma filter (Lee et al., 2009). We first determine the $98^{\text {th }}$ percentile of the double bounce class obtained from the temporal average of $T_{22}$ intensity image $\left(\frac{1}{n} \sum_{i=1}^{n}\left|S_{H H_{i}}-S_{V V_{i}}\right|^{2}\right)$ and single bounce class obtained from the temporal average of $T_{11}$ intensity image $\left(\frac{1}{n} \sum_{i=1}^{n}\left|S_{H H_{i}}+S_{V V_{i}}\right|^{2}\right)$. The $98^{\text {th }}$ percentile and a threshold of 5 pixels within a window of $3 \times 3$ are used to investigate if the target is a strong scatterer. Once the PS and DS patches are identified, adaptive spatial filtering of the objective pixel is performed within a $p \times p$ pixel window on the DS candidates only. The term spatial adaptive filtering mentioned throughout the manuscript refers to the selective averaging of pixels based on the identified SHP's. It includes generation of SHP's by using scattering properties and space adaptive averaging of DS pixels.

\subsubsection{Polarimetric Optimization}

Polarimetric optimization is a method to increase the interferometric phase quality and number of PS and DS involved in PSI by selecting the polarization state that is least affected by decorrelation (Mullissa et al., 2017b) (Iglesias et al., 2014) (Navarro-Sanchez et al., 2014). To this effect, we first define the polarimetric interferometric coherency matrix $Z$, containing both polarimetric and interferometric information. $Z$ is given as:

$Z=\left\langle K K^{\dagger}\right\rangle=\left[\begin{array}{cc}T_{i i} & \Omega_{i j} \\ \Omega_{i j}^{\dagger} & T_{j j}\end{array}\right]$, with $K=\left[\begin{array}{ll}\tilde{k}_{i}^{T} & \tilde{k}_{j}^{T}\end{array}\right]^{T}$.

where $T_{i i}$ and $T_{j j}$ are the coherency matrices related to images $i$ and $j$ and $\Omega_{i j}(i \neq j)$ is the polarimetric interferometric correlation matrix, respectively.

The interferometric coherence $\gamma_{i j}$ is formulated by projecting the scattering vectors $\tilde{k}_{i}$ and $\tilde{k}_{j}$ onto the complex unitary vector $\omega$,

$\gamma_{i j}=\left|\gamma_{i j}\right| e^{i \phi}=\frac{\omega^{\dagger} \Omega_{i j} \omega}{\sqrt{\left(\omega^{\dagger} T_{i i} \omega\right)\left(\omega^{\dagger} T_{j j} \omega\right)}}$.

Coherence optimization aims at finding the $\omega$ values that maximize the average coherence amplitude. The complex unitary projection vector $\omega$ can 
be represented with parameters that resemble the physical attributes of radar targets as shown in (5.6). Hence, it is parameterized as:

$\omega=\left[\begin{array}{c}\cos \alpha \\ e^{i \delta} \sin \alpha \cos \beta \\ e^{i \psi_{q}} \sin \alpha \sin \beta\end{array}\right]$

with $0 \leq \alpha \leq \pi / 2,0 \leq \beta \leq \pi,-\pi \leq \delta \leq \pi$ and $-\pi \leq \psi_{q} \leq \pi$.

For dual polarized data $\omega$ reduces to:

$\omega=\left[\begin{array}{c}\cos \alpha \\ e^{i \delta} \sin \alpha\end{array}\right]$.

The optimal scattering mechanism $\omega$ is obtained by numerical optimization of the four parameters described in (5.12) or the two parameters in (5.13) that provide the highest coherence amplitude in (5.11). Numerical optimization routines like the conjugate gradient descent method (Fletcher and Reeves, 1964) can be used to obtain the optimal parameter values.

\subsubsection{DS selection and processing}

In the traditional PSI analysis PS candidates are targeted for further analysis in the multi-temporal InSAR processing framework. These pixels correspond to a strong point scatterer by which all other secondary scatterers present within the resolution cell are dominated (Figure 5.1a). In contrast, DS candidates are characterized by a scattering mechanisms where none of the mechanisms dominate (Figure 5.1b and 5.1c). In practice, there can either be one type of scattering mechanisms in the resolution cell where none of the mechanisms dominates (Figure 5.1b) or it can be a mixture of different scattering mechanisms where some mechanisms are slightly dominant on the others (Figure 5.1c). If there is a mixture of scattering mechanisms within the resolution cell, phase decomposition (Cao et al., 2016) (Fornaro et al., 2015) can be applied to select the polarization state with the coherent scattering mechanism for application into the different multi-temporal InSAR analysis methods (Ferretti et al., 2011) (Hooper, 2008) (Perissin and Wang, 2012).

To characterize the differential phase vectors of DS pixels we first need to derive the interferometric coherence matrix $\Gamma$ (Ferretti et al., 2011). To this effect, we first extend the polarimetric optimization routine described in section 5.2.4 from a single baseline to a multi-baseline case to determine the optimal polarization state $\omega$. The polarimetric interferometric coherency matrix $Z$ discussed in (5.10) is expanded to the multi-baseline case as:

$Z=\frac{1}{N_{W}} \sum_{K \in \eta} K K^{\dagger}=\left[\begin{array}{ccc}T_{i i} & \cdots & \Omega_{i n} \\ \vdots & \ddots & \vdots \\ \Omega_{i n}^{\dagger} & \cdots & T_{n n}\end{array}\right]$, where $K=\left[\begin{array}{lll}{\tilde{k_{i}}}^{T} & \cdots & {\tilde{k_{n}}}^{T}\end{array}\right]^{T}$ 


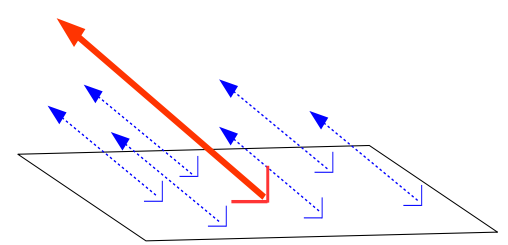

(a)

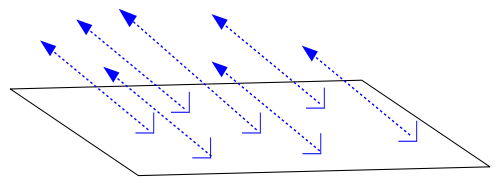

(b)

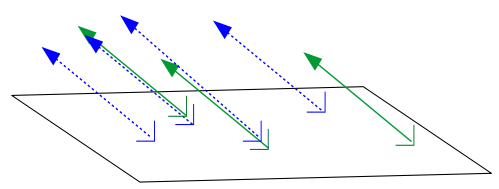

(c)

Figure 5.1: Pixel classes utilized in multi-temporal InSAR analysis (a) PS candidate (b) DS candidate with incoherent scattering mechanisms (c) DS candidate with coherent scattering mechanism.

Here $\eta$ represents a patch of homogeneous pixels defined in Section IIC containing $N_{W}$ pixels and $i \in[1,2, \ldots, n]$. In the multi-baseline case, the optimal polarization state $\omega$ is obtained by numerical optimization of the parameters described in (5.12) and (5.13) that provides the highest average coherence of the $\frac{n(n-1)}{2}$ set of interferograms. Therefore $\Gamma$ is constructed as:

$\Gamma=\left\langle s s^{\dagger}\right\rangle=\frac{1}{N_{W}} \sum_{s \in \eta} s s^{\dagger}=\left[\begin{array}{cccc}1 & \gamma_{12} & \cdots & \gamma_{1 n} \\ \gamma_{12}^{*} & 1 & \cdots & \gamma_{2 n} \\ \vdots & \vdots & \ddots & \vdots \\ \gamma_{1 n}^{*} & \gamma_{2 n}^{*} & \cdots & 1\end{array}\right]$

where $s=\left[\begin{array}{ccc}s_{i} & \cdots & s_{n}\end{array}\right]^{T}$ is the normalized polarimetric scattering coefficient of a stack of $n$ images which is derived as $s_{i}=\frac{I_{i}}{\sqrt{I_{i} I_{i}^{*}}}$ with $I_{i}=\omega^{\dagger} \tilde{k}_{i}$. $\Gamma$ is distinctly different from $T$ and $Z$ because it contains only interferometric information. $\Gamma$ is a Hermitian positive semi-definite matrix where each element corresponds to the complex interferometric coherence $\gamma_{i j}$, with the modulus representing the coherence amplitude and the argument representing the interferometric phase. The flat earth and topographic phase contribution are removed from the off diagonal components of $\Gamma$ prior to adaptive spatial 
filtering.

An eigenvalue decomposition routine is applied to decompose $\Gamma$ into its respective eigenvalues and eigenvectors using a singular value decomposition. It is given as:

$\Gamma=Q \Lambda Q^{*}=\sum_{i=1}^{n} \lambda_{i} q_{i} q_{i}^{\dagger}$

Here $Q=\left[q_{1} \cdots q_{n}\right]$ is the matrix of orthogonal eigenvectors arranged in columns and $\Lambda$ is the diagonal matrix containing non-zero eigenvalues $\lambda$ arranged in decreasing order. The eigenvectors represent the scattering mechanisms and the eigenvalues represent the statistical weight assigned to each scattering mechanism.

Hence, to describe the statistical disorder of each scatterer type the scattering entropy $H$ described in Section 5.2.2 is used. We modify the equation for $H$ to derive the statistical disorder of the phases $(\Psi)$ in a resolution cell as:

$\Psi=-\sum_{k=1}^{n} P_{k} \log _{n}\left(P_{k}\right)$ with $P_{i}=\frac{\lambda_{i}}{\sum_{k=1}^{n} \lambda_{k}}$ and $\sum_{k=1}^{n} P_{k}=1$.

For low $\Psi$ values the resolution cell is dominated by a deterministic point scatterer that dominates other scatterers. Therefore, the second and higher eigenvalues within the resolution cell may be neglected as noise sources. For strong point scatterers, such as PS candidates the SVD decomposition results in one non-zero eigenvalue representing the strong scatterer, indicating a single dominant scattering mechanism (Lee and Pottier, 2009). In this case, (5.16) reduces to $\Gamma=\lambda_{1} q_{1} q_{1}^{\dagger}$. Hence, for PS candidates the coherence matrix obtained from the dominant scattering mechanism $\Gamma\left(q_{1}\right)$ and the original coherence matrix are nearly identical i.e. $\Gamma \approx \Gamma\left(q_{1}\right)$. In practice, for entropy values closer to zero, the scattering media can be considered as deterministic and the dominant scattering matrix component can be extracted as the eigenvector corresponding to the largest eigenvalue (Cloude and Pottier, 1995) (Cloude, 1990). In this case, interference of secondary scatterers with the dominant scattering mechanism is insignificant and can be ignored. Hence, the optimal average coherence values can be achieved by discarding all secondary scattering mechanisms and the optimal coherence matrix $\Gamma\left(q^{*}\right)$ becomes $\Gamma\left(q^{*}\right)=\Gamma\left(q_{1}\right)$ (Cao et al., 2016).

For DS candidates with higher $\Psi$ two cases are distinguished. The first case is that $\Psi$ is close to 1 . This indicates that there are $n$ mutually orthogonal scattering mechanisms with similar amplitude. In this case the scattering can be characterized as a random noise process. Hence, it is considered a random DS and will yield a low coherence and the pixel is useless for multi-temporal InSAR analysis. The second case is that $\Psi$ achieves a moderate value. This 
indicates that there are $n$ dominant scattering mechanisms with decreasing dominance weights. Analysis of secondary scattering mechanisms and their differential phase interference pattern, however revealed that differential phases of some scattering mechanisms are in-phase. These scattering mechanisms are in-phase when they follow the same differential phase pattern indicating the same deformation pattern. Therefore, the coherence matrix adds up to a higher pixel coherence amplitude. Hence, by adaptively selecting only the scattering mechanisms with differential phases that are in-phase with the primary scattering mechanism we can maximize the amplitude of the coherence sum. Even for very low entropy values however some of the secondary scattering mechanisms may interfere constructively thus providing an incremental improvement to the coherence. However, the coherence from the strong scatterer is already high (Figure 5.2a, 5.2c, 5.3a and 5.3c), higher than the quality threshold imposed on multi-temporal InSAR processing. For computational efficiency it is suggested to select a threshold for entropy values where the secondary scattering mechanisms are significant enough to increase the coherence above the threshold values. The selection of these threshold values should be evaluated on a case to case basis. The adaptive selection of secondary scattering mechanisms that interfere constructively is achieved by first defining an arbitrary interferometric coherence matrix, $\Gamma_{c}$ constructed from of the subset of eigenvectors as:

$\Gamma_{c}=\sum_{i \in c} \lambda_{i} q_{i} q_{i}^{\dagger}$ where $c \subset\{1,2, \ldots, n\}$

Here $c$ is the subset of the eigenvectors. Since, the coherence matrices are Hermitian positive semi-definite matrices of which the strictly upper triangular elements represent unique values for the interferometric phase. Hence, we use only the $\frac{n(n-1)}{2}$ strictly upper triangular elements of the coherence matrix denoted by [.] in the optimization routine. The optimization criterion for selecting the constructively interfering scattering mechanisms is the amplitude of the sum of complex coherence values given as:

$\epsilon=\left|\sum_{b=2}^{n} \sum_{a=1}^{b-1}\left[\gamma_{a b}\right]\right|$

Here $a$ and $b$ are the row and column number of the elements in $\Gamma_{c}$ and $\epsilon$ is the amplitude of the sum of the upper triangular coherence values in $\Gamma_{c}$. The optimal set of eigenvectors $c_{o p t}$ selected by discarding the unstable scattering mechanisms is given by:

$c_{o p t}=\arg \max _{c}\{\epsilon\}$. 


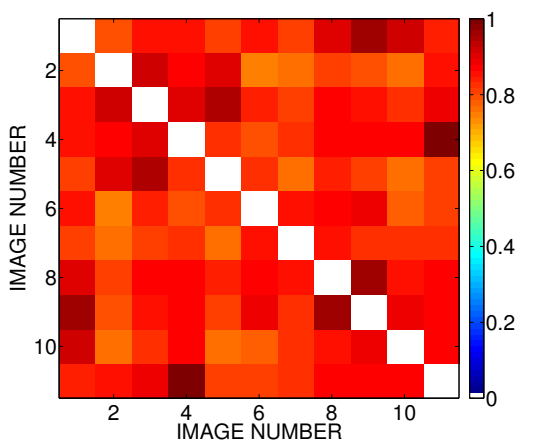

(a)

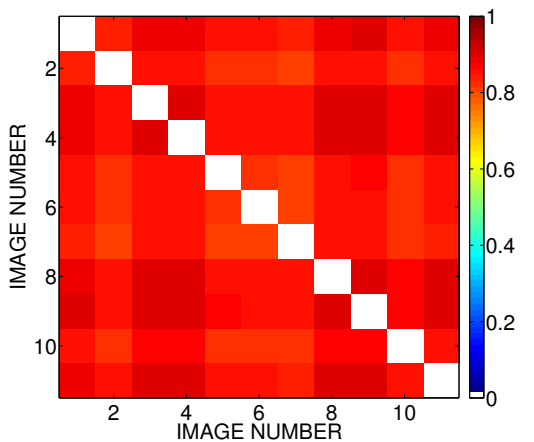

(c)

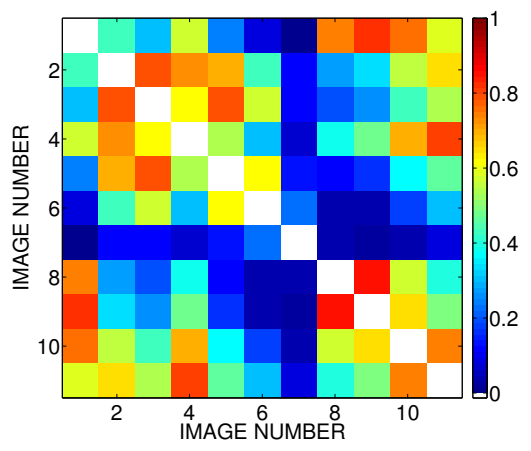

(b)

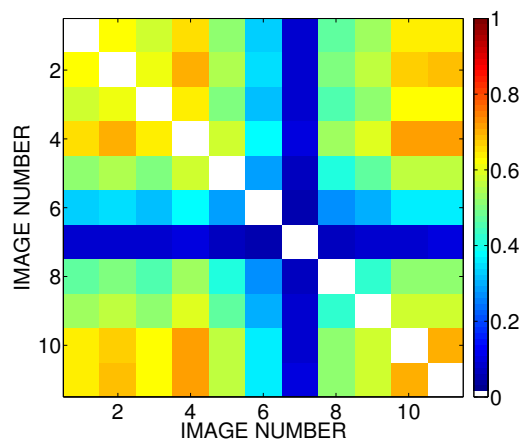

(d)

Figure 5.2: Absolute value of the polarimetric interferometric coherence matrix extracted from ALOS PALSAR data (a) PS candidate for $\Gamma$ (b) DS candidate for $\Gamma$ (c) PS candidate derived from adaptive selection of constructively interfering scattering mechanisms $\Gamma\left(q^{*}\right)$ (d) DS candidate derived from adaptive selection of constructively interfering scattering mechanisms $\Gamma\left(q^{*}\right)$. For the PS candidate the coherence amplitude is high in all interferograms in both $\Gamma$ and $\Gamma\left(q^{*}\right)$. For the DS candidates (b) and (d) it can clearly be seen that coherence improves in almost all interferograms except in images six and seven where the effect of baseline decorrelation occurs. 


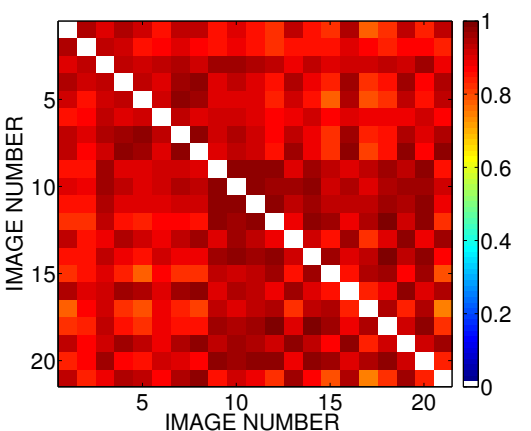

(a)

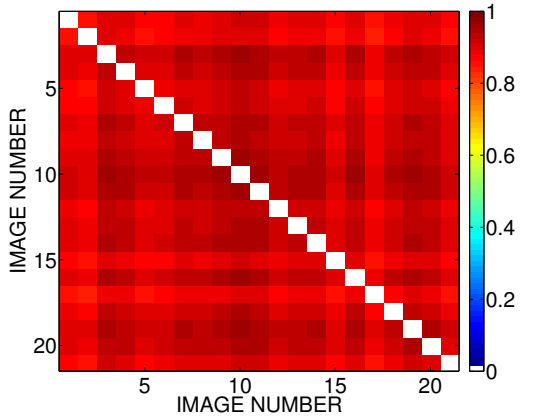

(c)

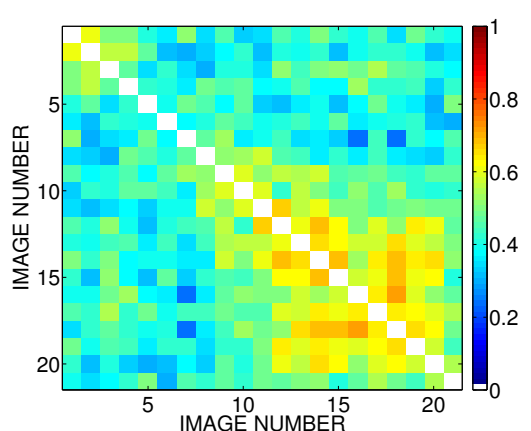

(b)

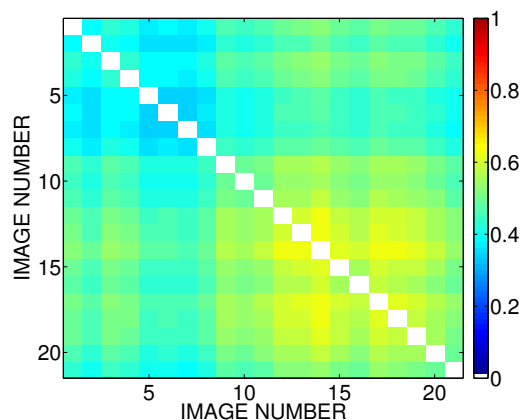

(d)

Figure 5.3: Absolute value of the polarimetric interferometric coherence matrix extracted from Sentinel-1 data (a) PS candidate for $\Gamma$ (b) DS candidate for $\Gamma$ (c) PS candidate derived from adaptive selection of constructively interfering scattering mechanisms $\Gamma\left(q^{*}\right)$ (d) DS candidate derived from adaptive selection of constructively interfering scattering mechanisms $\Gamma\left(q^{*}\right)$.

and the optimal coherence matrix $\Gamma\left(q^{*}\right)$ calculated from the selected optimal subset of eigenvectors is given as:

$\Gamma\left(q^{*}\right)=\sum_{i \in c_{o p t}} \lambda_{i} q_{i} q_{i}^{\dagger}$

As is evident from (5.20) the amplitude of the coherence sum is maximized by using an exhaustive search optimization. This can be computationally expensive if a large stack of images is being processed.

The proposed method work flow is summarized as:

1. Apply the scattering property based adaptive spatial filter to identify and filter DS.

2. Optionally: implement coherence optimization to derive the optimal polarization state $\omega$. 


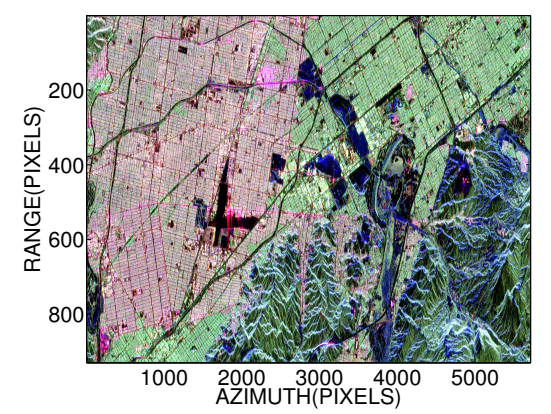

(a)

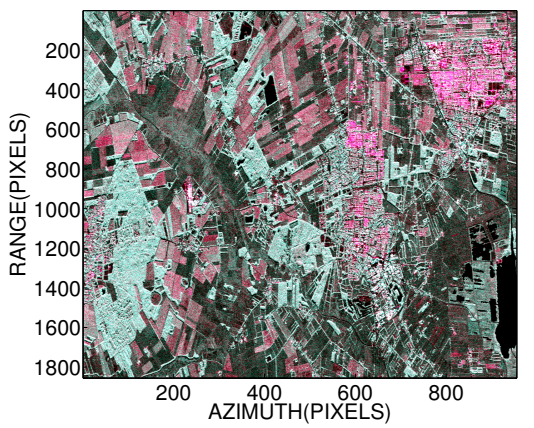

(b)

Figure 5.4: Location map of the two test areas. (a) Intensity image obtained by incoherent temporal average of 11 ALOS PALSAR images of San Fernando valley, California. Blue stands for $\left|S_{H H}+S_{V V}\right|^{2}$, green stands for $\left|S_{H H}-S_{V V}\right|^{2}$ and red stands for $\left|2 S_{V H}\right|^{2}$. (b) Intensity image obtained by incoherent temporal average of 21 Sentinel-1 images of the Groningen area, The Netherlands. Blue stands for $\left|S_{V V}+S_{V H}\right|^{2}$, green stands for $\left|S_{V H}\right|^{2}$ and red stands for $\left|S_{V V}\right|^{2}$.

3. Derive the scattering entropy $\Psi$ by applying target decomposition to polarimetric interferometric coherence matrix $\Gamma$ and select the target DS by applying a threshold to $\Psi$.

4. Adaptively select constructively interfering scattering mechanism of the selected DS by maximizing the amplitude of the complex coherence sum in the polarimetric interferometric coherence matrix $\Gamma$.

5. Integrate the optimal coherence matrix $\Gamma\left(q^{*}\right)$ into the Quasi-Permanent scatterer (QPS) interferometry multi-temporal InSAR analysis.

The QPS technique differs from the PSI technique in three aspects. Interferograms are filtered and are not required to be formed from a single common master image and the target height and displacement are estimated by selecting a subset of interferograms. In this way, it inserts the coherence value as a weight to make sure that only coherent interferograms in $\Gamma$ influence the result of estimation. Hence, no phase triangualtion is required to return to $n-1$ interferograms as in the SqueeSAR (Ferretti et al., 2011). The QPS method is detailed by Perissin and Wang (2012).

\subsection{Datasets}

To evaluate the performance of the proposed method 11 quad polarized ALOS PALSAR images acquired from June 8, 2006 to March 16, 2009 over the San Fernando valley, California (Table 5.2) and 21 dual polarized Sentinel-1 images acquired from August 14, 2015 to April 05, 2017 over the Groningen area in the Netherlands (Table 5.2) were used. The ALOS PALSAR 
sensor acquires data in $\mathrm{L}$ band for the quad polarization images whereas the Sentinel-1 sensor acquires data in $\mathrm{C}$ band for both dual and single polarimetric images (Table 5.1). The test areas contain $930 \times 5715$ pixels and cover $10.76 \mathrm{~km} \times 20.23 \mathrm{~km}$ for the ALOS PALSAR and $1855 \times 955$ pixels and $13.3 \mathrm{~km} \times 6.8 \mathrm{~km}$ for the Sentinel-1 data in range and azimuth directions, respectively. The ALOS PALSAR image consists of predominantly urban region (Figure 5.4a) and the Sentinel-1 image consists of a predominantly natural environment that consists of agricultural area (Figure 5.4b). The polarimetric content of the two scenes can be observed from the false color composites of the two regions (Figure 5.4).

\begin{tabular}{lcc}
\hline & Quad polarized data & Dual polarized data \\
\hline Sensor & ALOS-PALSAR & Sentinel-1A and $1 \mathrm{~B}$ \\
Resolution & $11.38 \mathrm{~m} \times 3.54 \mathrm{~m}$ & $3.67 \mathrm{~m} \times 13.94 \mathrm{~m}$ \\
Incidence angle & $23.6^{0}$ & $39.35^{0}$ \\
Orbit & Ascending & Ascending \\
Temporal baseline & 46 days & 6 days \\
Dates & $2006 / 06 / 08-2009 / 03 / 16$ & $2015 / 08 / 14-2017 / 04 / 05$ \\
Number of images & 11 & 21 \\
\hline
\end{tabular}

Table 5.1: Acquisition parameters for the ALOS PALSAR quad polarized images and Sentinel-1 dual polarized images.

\subsection{Results}

Pixel similarity within a pixel neighborhood is established by implementing the Wishart distance classification method (Lee et al., 1999a). The average coherency matrix is first constructed by averaging the single look coherency matrices in the temporal dimension. The scattering property for the scene i.e. average scattering mechanism $(\tilde{\alpha})$ and the scattering entropy $(H)$ are derived by implementing the Cloude-Pottier eigenvalue-eigenvector decomposition method (Cloude and Pottier, 1997). The $\tilde{\alpha}$ derived in section IIB by implementing the Cloude-Pottier eigenvalue-eigenvector decomposition assumes different values from the optimal scattering mechanism $\alpha$ defined in this section. The reason is that $\tilde{\alpha}$ describes the dominant scattering mechanism present within the resolution cell whereas $\alpha$ defines the scattering mechanism that maximizes the interferometric coherence in a polarimetric SAR data stack. Hence, $\tilde{\alpha}$ and $\alpha$ have similar values in resolution cells where there is a deterministic point scatterer. In DS scatterers $\tilde{\alpha}$ and $\alpha$ assume different values. To implement the Wishart distance classification routine for the classification of scattering properties, $\tilde{\alpha}$ and $H$ values were plotted on the $H / \alpha$ plane and the center points in each class selected to initialize the classification process. We implemented combination of the unsupervised target decomposition classifier and the supervised Wishart classifier routine described in section IID which resulted in eight distinct scattering mechanism 


\begin{tabular}{lcccccc}
\hline \multicolumn{5}{c}{ Sentinel-1 } & \multicolumn{3}{c}{ ALOS PALSAR } \\
\hline Number & Date & PB & TB & Date & PB & TB \\
\hline 1 & $2015 / 08 / 14$ & -61.91 & -336 & $2006 / 06 / 08$ & $1,121.70$ & -322 \\
2 & $2015 / 08 / 26$ & -3.01 & -324 & $2006 / 09 / 08$ & -730.50 & -230 \\
3 & $2015 / 09 / 07$ & 79.57 & -312 & $2007 / 03 / 11$ & $-1,077.72$ & -46 \\
4 & $2015 / 10 / 13$ & -69.05 & -276 & $2007 / 04 / 26$ & 0 & 0 \\
5 & $2016 / 07 / 15$ & 0 & 0 & $2007 / 07 / 27$ & $-1,322.48$ & 92 \\
6 & $2016 / 07 / 27$ & 41.05 & 12 & $2007 / 10 / 27$ & $-2,241.96$ & 182 \\
7 & $2016 / 08 / 08$ & 24.54 & 24 & $2008 / 01 / 27$ & $-2,730.32$ & 276 \\
8 & $2016 / 10 / 07$ & 21.50 & 84 & $2008 / 09 / 13$ & $1,629.91$ & 506 \\
9 & $2016 / 10 / 19$ & -44.09 & 96 & $2008 / 10 / 29$ & $1,351.22$ & 552 \\
10 & $2016 / 10 / 31$ & -92.42 & 108 & $2009 / 01 / 29$ & 655.61 & 644 \\
11 & $2016 / 11 / 12$ & -62.28 & 120 & $2009 / 03 / 16$ & 67.30 & 690 \\
12 & $2016 / 11 / 24$ & -12.54 & 132 & & & \\
13 & $2016 / 12 / 06$ & 31.22 & 144 & & & \\
14 & $2016 / 12 / 18$ & 9.14 & 156 & & & \\
15 & $2016 / 12 / 30$ & -70.22 & 168 & & & \\
16 & $2017 / 01 / 05$ & 8.34 & 174 & & & \\
17 & $2017 / 01 / 23$ & -38.31 & 192 & & & \\
18 & $2017 / 02 / 04$ & -5.47 & 204 & & & \\
19 & $2017 / 02 / 16$ & -11.92 & 216 & & & \\
20 & $2017 / 03 / 06$ & -8.72 & 234 & & & \\
21 & $2017 / 04 / 05$ & 20.32 & 264 & & & \\
& & & & &
\end{tabular}

Table 5.2: List of Sentinel-1 and ALOS PALSAR data used in this chapter. In the table PB refers to perpendicular baseline in meters whereas TB refers to temporal baseline in days.

classes. A $10 \%$ pixel switching threshold is used as termination criteria. To establish homogeneity within the neighboring of DS pixels, spatial contiguousness of pixels of a similar scattering class is assessed. A minimum number of eight pixels within a $15 \times 15$ pixels is used to establish this pixel neighborhood. Pixels that did not fulfill the eight interconnected neighboring pixels were designated as isolated pixels. Isolated pixels not belonging to the PS candidates were merged with the class that has the majority surrounding those pixels. The number of homogeneous pixels generated using a $15 \times 15$ pixel neighborhood from the classified scattering mechanism is shown in Figures 5.5a and 5.6a. The effectiveness of the scattering property based adaptive filtering method is demonstrated by filtering a single look Sentinel-1 image (Figure 5.7). The scattering property based adaptive filtering method clearly reduces speckle while preserving feature shapes and edges.

The proposed method adaptively selected the secondary scattering mechanisms that interfere constructively with the dominant scattering mechanisms hence, improving the overall pixel coherence. This can be observed from Figures 5.2 and 5.3. The interferograms with short temporal baselines located near the diagonal line show higher coherence. However, interferograms with 


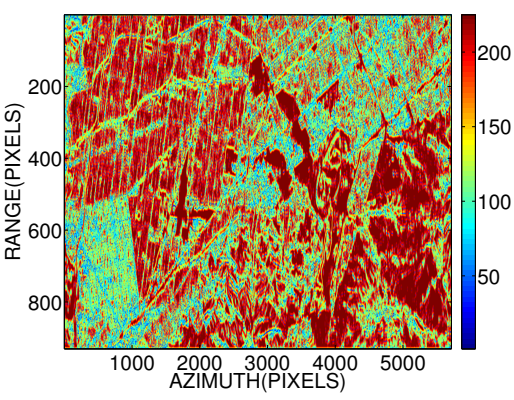

(a)

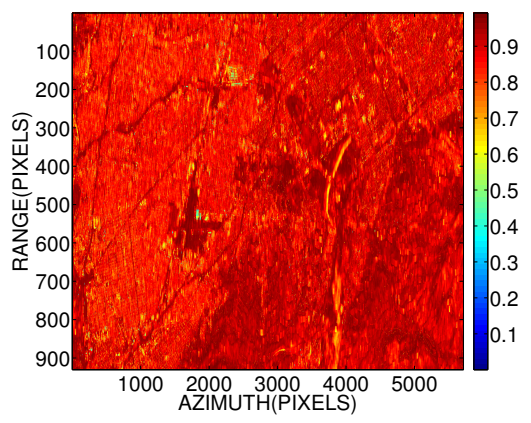

(b)

Figure 5.5: (a) Number of homogeneous pixels identified within $15 \times 15$ window by the classification of scattering mechanisms for the ALOS PALSAR data. (b) Scattering entropy for the study area. Low entropy values represent a deterministic single dominant scattering mechanisms whereas high entropy values represent multiple random scattering mechanisms within a resolution cell.

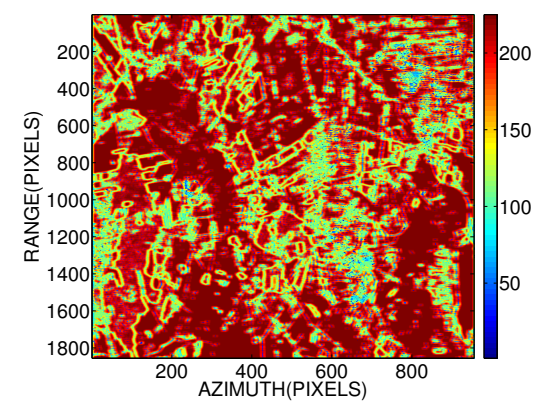

(a)

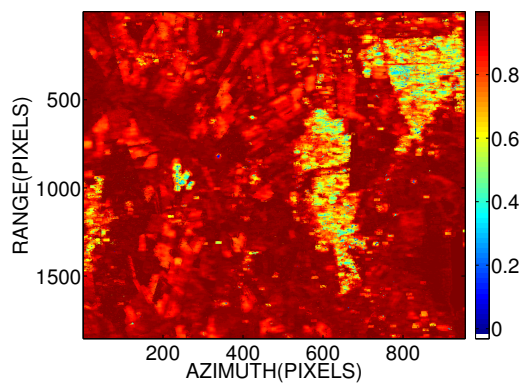

(b)

Figure 5.6: (a) Number of homogenous pixels identified within $15 \times 15$ window by the classification of scattering mechanisms from Sentinel-1 data. (b) Scattering entropy for the study area.

long perpendicular baselines (in images 6 and 7 of Figure 5.2b and 5.2d) display low coherence values. With DS decomposition the coherence values with short temporal baselines show a significant increase $(>0.1)$ whereas the coherence interferograms with long spatial and temporal baselines do not show much improvement from the polarimetrically optimized coherence matrix $\Gamma_{\omega}$. This is reasonable because with temporal decorrelation, the scattering property of the media changes and that may be un-related to interference of different scattering mechanisms.

We selected the DS candidate points for the application of the proposed method by using the phase entropy $\Psi$ defined in section 5.2.5. For $\Psi \leq 0.4$, the dominant scattering mechanism $q_{1}$ achieves the highest coherence value indic- 


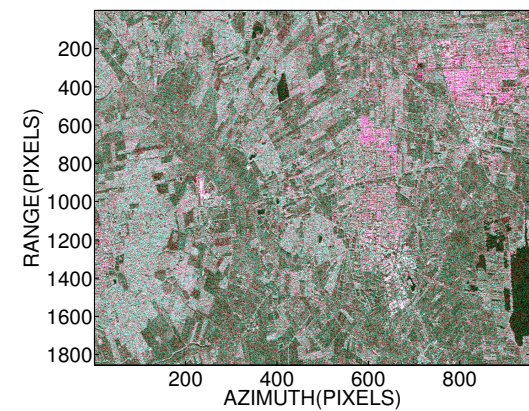

(a)

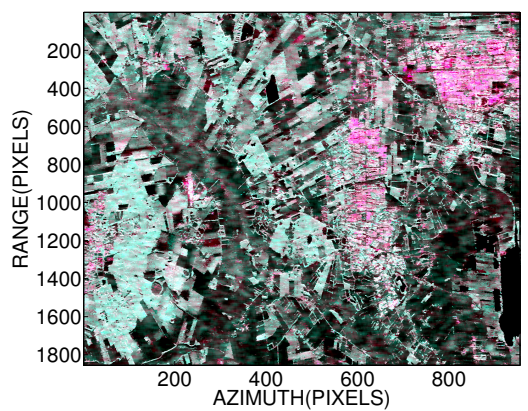

(b)

Figure 5.7: Comparison between (a) unfiltered Sentinel-1 dual polarization intensity image acquired on 2015/08/14 and (b) Intensity of the same image obtained by applying the scattering property based adaptive filtering method. It can clearly be seen that the proposed method reduces speckle effectively. In the images blue stands for $\left|S_{V V}+S_{V H}\right|^{2}$, green stands for $\left|S_{V H}\right|^{2}$ and red stands for $\left|S_{V V}\right|^{2}$.

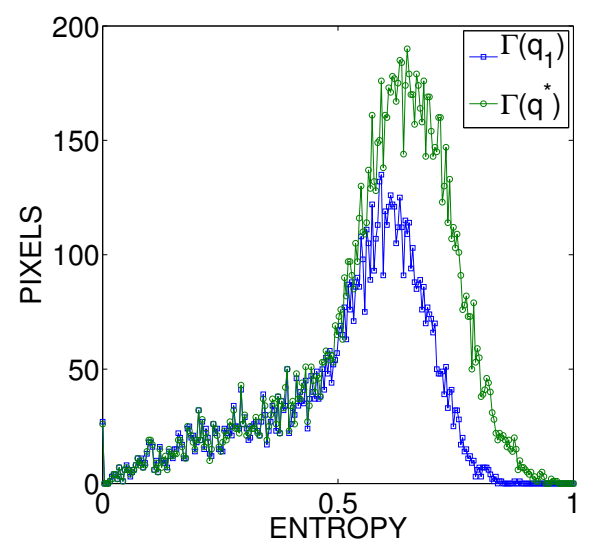

Figure 5.8: Comparison of entropy distribution for pixels with temporal coherence greater than 0.9 for the Sentinel-1 data. For $\Psi>0.4$ selecting the constructively interfering secondary scattering mechanisms with the dominant scattering mechanism $\Gamma\left(q^{*}\right)$ selects more pixels than by using the dominant scattering mechanism $\Gamma\left(q_{1}\right)$.

ating all secondary scattering mechanisms constitute noise. For $0.4<\Psi \leq 0.9$, the proposed method increased the average pixel coherence by removing the noisy scattering mechanism (Figure 5.8).

Results from the proposed method were evaluated in terms of the number of pixels selected as measurement points i.e. the detail of the derived displacement map and the accuracy of the derived displacement map. We used the dominant scattering mechanism $q_{1}$ derived from the VV channel $\Gamma\left(q_{1}\right)$ 
selected by implementing the method detailed in (Cao et al., 2016) and applied the proposed method on the polarimetrically optimized coherence matrix $\Gamma_{\omega}\left(q^{*}\right)$ for comparison. For consistency, adaptive spatial filtering in the formation of $\Gamma_{V V}, \Gamma\left(q_{1}\right)$ and $\Gamma_{\omega}\left(q^{*}\right)$ used the same SHP patch. On the ALOS PALSAR data $\Gamma_{V V}$ selected 8,091 pixels, $\Gamma\left(q_{1}\right)$ selected 21,476 pixels and $\Gamma_{\omega}\left(q^{*}\right)$ selected 63,605 pixels, respectively by using a temporal coherence threshold $\gamma_{t}>0.9$ (Table 5.3). The temporal coherence is the parameter that shows the variations in phase residuals between the model and observed phases after estimation and removal of atmospheric phase screen, orbital and DEM phase errors (Ferretti et al., 2001). To evaluate the improvement obtained when using polarimetric optimization we compared the number of pixels selected from polarimetrically optimized coherence matrix $\Gamma_{\omega}$ and dominant scattering mechanism derived from decomposition of optimized coherence matrix $\Gamma_{\omega}\left(q_{1}\right) . \quad \Gamma_{\omega}$ selected 28,264 pixels and $\Gamma_{\omega}\left(q_{1}\right)$ selected 42,654 pixels.

\begin{tabular}{lcc}
\hline & ALOS PALSAR & Sentinel-1 \\
\hline PSI & - & $2,234(0.12 \%)$ \\
$\Gamma_{V V}$ & $8,091(0.15 \%)$ & $5,244(0.29 \%)$ \\
$\Gamma_{\left(q_{1}\right)}$ & $21,476(0.4 \%)$ & $8,639(0.48 \%)$ \\
$\Gamma_{\omega}\left(q^{*}\right)$ & $63,605(1.19 \%)$ & $13,578(0.76 \%)$ \\
\hline
\end{tabular}

Table 5.3: Number of pixels selected from the coherence matrix derived from VV channel $\Gamma_{V V}$, the dominant scattering mechanism $\Gamma\left(q_{1}\right)$ and adaptively selecting the constructively interfering scattering mechanisms from the polarimetrically optimized coherence matrix $\Gamma_{\omega}\left(q^{*}\right)$.

Next, the proposed method was implemented on a dual polarized Sentinel-1 data and the VV channel. The $\Gamma_{V V}$ selected 5,244 measurement points, $\Gamma\left(q_{1}\right)$ selected 8,639 pixels and $\Gamma_{\omega}\left(q^{*}\right)$ selected 13,578 pixels, respectively, by using a temporal coherence threshold $\gamma_{t}>0.9$. To evaluate the improvement obtained when using polarimetric optimization we compared the number of pixels selected from polarimetric optimization, dominant scattering mechanism derived from optimized coherence matrix and the proposed method. The polarimetrically optimized coherence matrix $\Gamma_{\omega}$ selected 8,087 pixels, dominant scattering mechanism $\Gamma_{\omega}\left(q_{1}\right)$ selected 9,976 pixels. The ALOS PALSAR scene is located in a predominantly urban region where there is a mixture of distinct scattering mechanisms as shown in Figure 5.5b. Therefore, the proposed method was able to improve the pixel average coherence significantly (Figure 5.9). The Sentinel-1 image however in contrast is located in a rural region with more random scattering mechanisms and a high entropy (Figure 5.6b). Hence the proposed method did not provide much improvement in coherence.

The overall computational burden of the proposed method is $6.35 \times 10^{-3} \mathrm{~s}$ per DS candidate pixel. The implementation of the scattering property based adaptive filtering routine required $23 \%$ of the overall computational time 


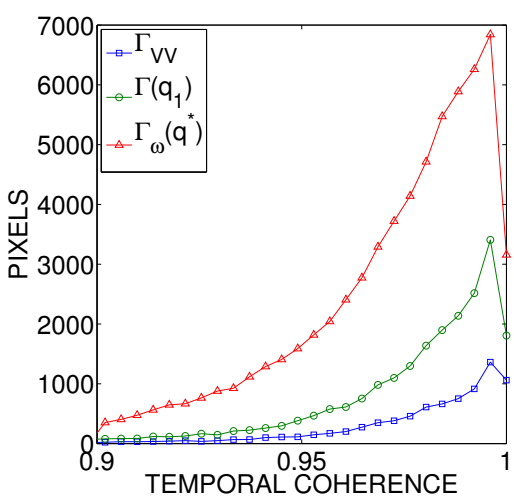

(a)

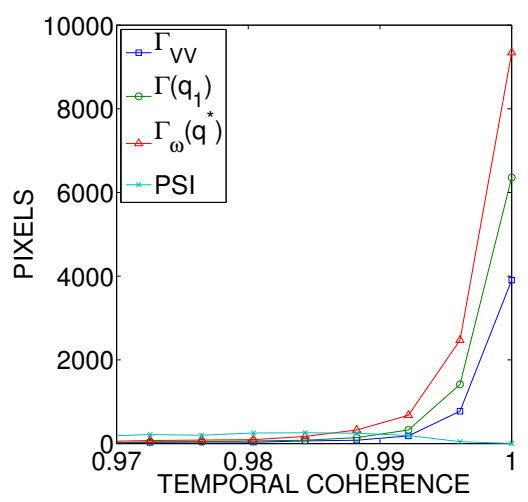

(b)

Figure 5.9: Temporal coherence estimated from the (a) ALOS PALSAR dataset (b) Sentinel-1 dataset

per pixel, whereas polarimetric coherence optimization utilizing conjugate gradients required $31 \%$ per pixel. However, the phase entropy based DS identification and the coherence matrix optimization by selection of coherent scattering mechanism by exhaustive search optimization was computationally expensive especially if the full set of eigenvectors was considered for optimization. It required $3.42 \times 10^{-3}$ s per DS pixel for the ALOS PALSAR data (11 images) and 8.98s per DS pixel for the Sentinel-1 data (21 images) when implemented in Matlab running on a Linux operating system with an Intel core i7 $2.70 \mathrm{GHz}$ processor laptop with 8 processing cores. It is clear that DS identification with an exhaustive search optimization of constructively interfering scattering mechanism is the most expensive. Its computational efficiency is significantly improved by imposing a threshold on the minimum eigenvalue for the eigenvector to be considered for optimization. It required $46 \%$ of the overall processing time if a threshold of 0.2 is applied on the eigenvalue when processing the Sentinel-1 data.

The deformation maps derived from the proposed method were compared with the VV channel and the dominant scattering mechanism derived from it. We derived the displacement maps shown in Figure 10 and 11 using SARPROZ multi-temporal InSAR processing software (Perissin et al., 2011). The deformation trend estimated from each method showed good agreement. However, the details provided by the proposed method is higher than $\Gamma_{V V}$ and $\Gamma\left(q_{1}\right)$. In the ALOS PALSAR scene more details are provided by the proposed method than by the other methods thereby clearly delineating the boundaries of the subsidence phenomena (Figure 5.10c). We have not included the results of PSI for the ALOS PALSAR dataset since a minimum number of 15-20 images is required for a PSI analysis (Crosetto et al., 2016).

To assess the performance of the displacement estimation from the proposed method, we compared the temporal coherence from $\Gamma_{V V}, \Gamma\left(q_{1}\right)$, and $\Gamma_{\omega}\left(q^{*}\right)$ 


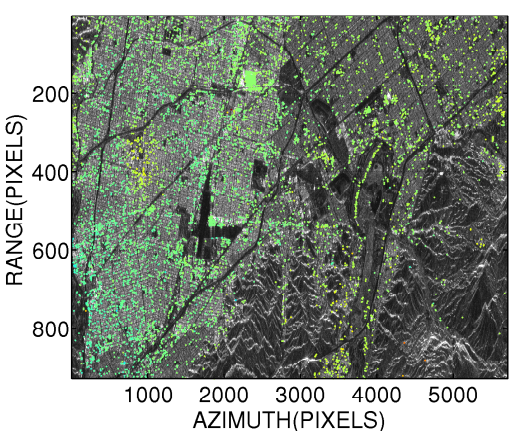

(a)

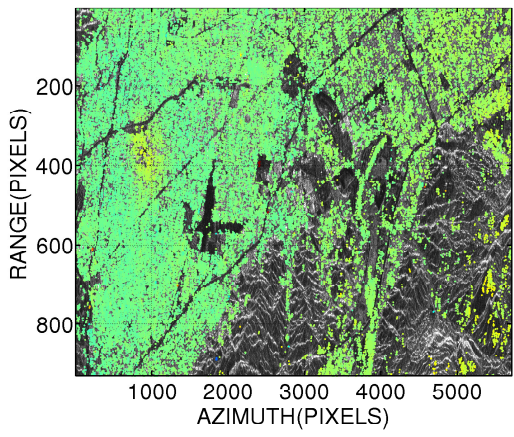

(c)

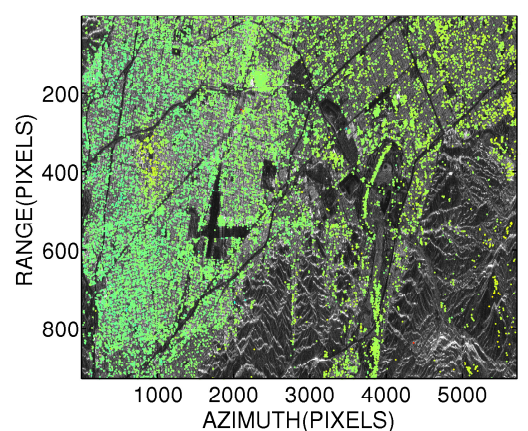

(b)

Figure 5.10: The deformation rate in $\mathrm{mm} \mathrm{yr}^{-1}$ measured from the adaptively filtered ALOS PALSAR images (a) VV channel $\Gamma_{V V}$ (b) Dominant scattering mechanism derived from VV channel $\Gamma\left(q_{1}\right)$ (c) Proposed method $\Gamma_{\omega}\left(q^{*}\right)$. The PSI method is not included because PSI analysis derived from 11 images is unreliable.

coherence matrices. Higher temporal coherence corresponds with a lower phase residual and a high model fit. It can be seen from Figure 5.9, $\Gamma_{\omega}\left(q^{*}\right)$ selects more pixels with high temporal coherence than $\Gamma_{V V}$ and $\Gamma\left(q_{1}\right)$. In this way, we conducted a detailed time series analysis around the village of Langelo at a location of a gas extraction plant and the town of Roden for the Sentinel-1 data (Figure 5.11). Figure 5.12a shows that the VV channel, $\Gamma\left(q_{1}\right)$ and $\Gamma_{\omega}\left(q^{*}\right)$ provide similar displacement time series when compared to a PS candidate. However, the accuracy of displacement measurement deviated when comparing a PS candidate and a nearby DS candidate (Figure $5.12 \mathrm{~b}$ ). The reason for this can be a combination of effect of filtering of DS candidates, the full resolution differential phase values that deviate from each other, and the variation in point density which induces unwrapping errors. Figure 5.12b is extracted over the Langelo gas extraction site in Groningen which is undergoing ground subsidence at a rate of $15 \mathrm{~mm}$ per year by the proposed method. A deformation rate of $18 \mathrm{~mm}$ per year reported by previous observation (ESA, 2016) agrees well with the measurement using the proposed method. 


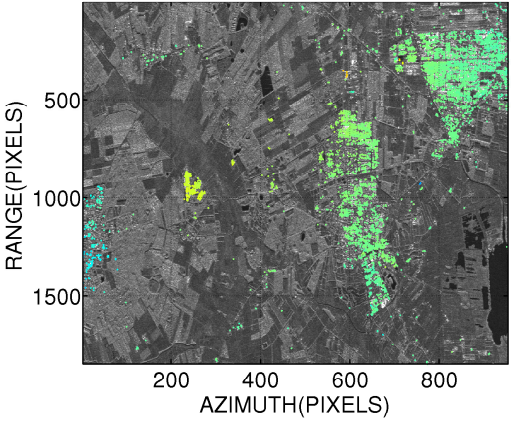

(a)

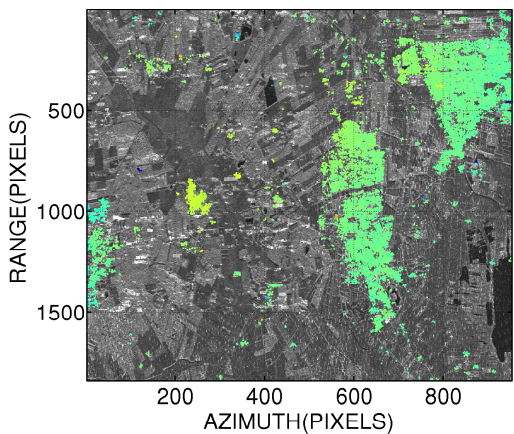

(c)

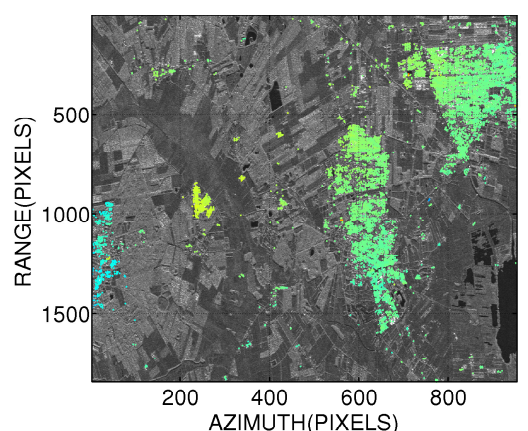

(b)

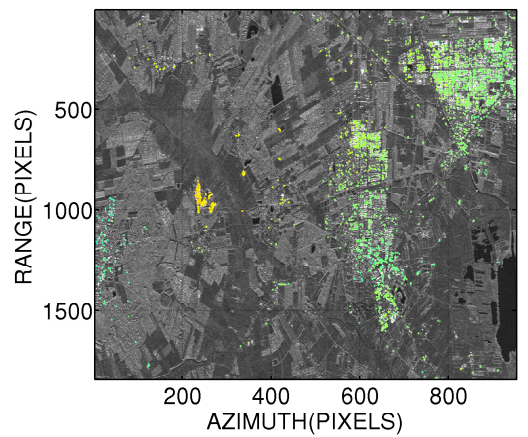

(d)

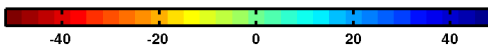

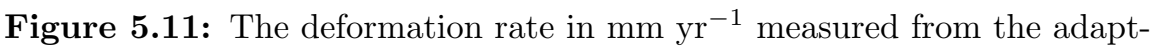
ively filtered Sentinel-1 images (a) VV channel $\Gamma_{V V}$ (b) Dominant scattering mechanism derived from VV channel $\Gamma\left(q_{1}\right)$ (c) Proposed method $\Gamma_{\omega}\left(q^{*}\right)$. (d) PSI

\subsection{Discussion and conclusion}

In this chapter, the scattering property based speckle filtering suggested by (Lee et al., 2006) (Mullissa et al., 2017a) for single quad polarized data has been extended to filter both quad polarized and dual polarized interferometric coherency matrices. Estimation of the polarimetric coherency matrix by averaging in the temporal dimension helped to preserve the scene resolution. This was important in an application to multi-temporal InSAR analysis, where most of the analysis is concentrated on pixel candidates that are coherent scatterers. The application of adaptive filtering also helped to improve the the estimate of coherence, as coherence bias depends upon the number of samples (looks) and the stationarity assumption used in estimation (Touzi et al., 1999). In this way, maintaining the homogeneity of the neighborhood 


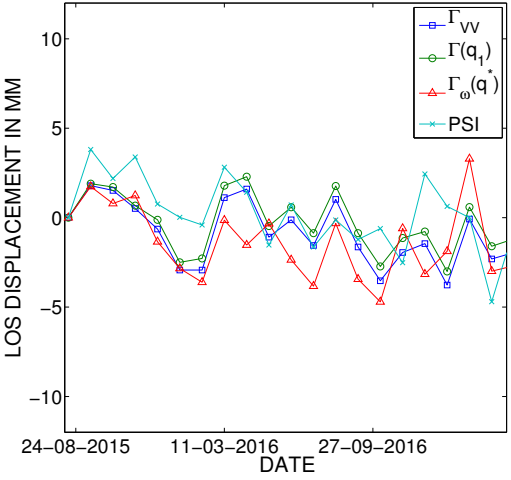

(a)

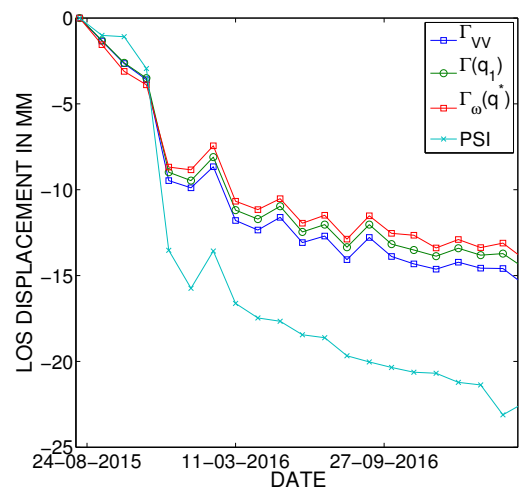

(b)

Figure 5.12: Displacement time series obtained from the Sentinel-1 datasets by applying different methods on (a) a measurement point from a PS candidate and (b) measurement point taken from a PS pixel candidate and a DS pixel candidate .

pixels by applying the scattering property based adaptive filtering and using a larger averaging window to have a large number of samples minimizes the bias in coherence estimation.

The modified phase decomposition method described in this manuscript helped improving the interferometric phase quality by discarding incoherent scattering mechanisms in the resolution cell. The scattering mechanisms can be understood as scatterers that have distinct phase centers with different height and spatial locations. We exploited the interference pattern of the secondary scattering mechanisms to improve the coherence estimate of the resolution cell. It was effective in removing secondary scattering mechanisms that interfered with other dominant scattering mechanisms. Theoretically all secondary scattering mechanisms can be coherent but only a few of these scattering mechanisms do so, the rest constitutes noise. Its effect is more pronounced in DS pixels without a significantly dominant scatterer. Hence, the proposed method is removing these sources of noise from the pixel. 

Synthesis

6 


\subsection{Research findings and conclusions}

This chapter reviews the main elements of the thesis. It summarizes how the aims and objectives of the thesis were achieved. At the end, it provides suggestions and recommendations for future direction of this research. Detailed conclusions for the individual objectives are presented. This chapter summarizes the general conclusions from the thesis and discuss the key findings of this research in relation to the research objectives.

- Objective 1: To evaluate the improvements from polarization optimization methods in a natural environment.

In this objective, I investigated if the phase quality can be reliably improved by applying polarimetric optimization and coherence decomposition to PolSAR data acquired over an arid natural environment.

I applied three coherence optimization techniques and compared the coherence improvement for fully and partially polarized ALOS PALSAR data. For both dual polarized and quad polarized SAR data the equal scattering mechanism (ESM) proved to be the best performing coherence optimization method. In addition, we applied coherence decomposition to understand the effect of polarimetric optimization in single and double phase center scenarios representing a bare surface and vegetated regions. In single phase center scenarios polarization played a direct role in increasing the signal to noise ratio of the backscattered signal. In double phase center scenarios polarization increased the signal to noise ratio but the phase center location of the optimal polarization state was arbitrary. In some cases it was at the top of a canopy whereas in other cases it was located at the ground surface. The $\mathrm{HH}-\mathrm{VV}$ polarization channel did not provide the highest interferometric coherence but it consistently yielded a phase center closer to the ground.

Furthermore, I assessed the coherence and interferometric phase bias introduced from using all three optimizers as a function of number of looks. Joint diagonalization based coherence optimizer had the highest phase bias if the coherence matrix at each end of the baseline deviated from the polarimetric stationarity assumption. ESM is the optimizer with the least bias when using a large number of looks. To confirm the improvement of the interferometric phase quality from the different polarimetric optimization methods I compared the LOS displacements obtained from each optimizer with that of terrestrial GPS stations. ESM provided the highest time series correlation coefficient when compared with the other optimization methods even though DEM, orbital and atmospheric phase errors were not rectified. The reasons were that ESM explores the full polarization space and that it handles polarimetric stationarity by taking the average of coherency matrices in the multi-spatial and multi-temporal baselines. 
It is concluded that ESM is the best optimization method with the highest coherence and interferometric phase accuracy when applied in a single phase center scenario even though it is computationally the most expensive one. When using the dual polarization configuration the co-polarization channel (HH VV) achieved higher interferometric phase accuracy than the cross polarization channel (HH HV) and both performed better than the traditional $\mathrm{HH}$ channel. This was because the HV signal in the image was from vegetation and contained less signal to noise ratio than the VV channel. Therefore the polarimetric optimization methods could not improve the interferometric coherence estimate.

The following research question has been answered:

- Can polarimetric coherence optimization be used to reliably improve the quality of deformation measurement in a natural environment?".

Polarimetric optimization methods significantly improve the interferometric coherence estimation when compared with the single channel SAR interferometry. The reason for this was polarimetric datasets expand the polarimetric observation space. As discussed in Chapter 2, a polarization state in the linear basis can be transformed to any elliptical or circular basis. This expansion of "observation space" improves the interferometric coherence at the cost of computational time. The higher interferometric coherences is associated with higher interferometric phase accuracy therefore leading to a more accurate estimation of deformation. This was supported by both the simulated experiments and the in-situ GPS comparisons.

- To exploit polarimetric diversity in PolSAR data to select statistical homogeneous pixels (SHP's) that is suitable for estimating the coherency matrix.

In this objective, I investigated if the polarimetric scattering mechanisms can be used to select statistically homogeneous pixels for the estimation of the coherency matrix. In addition, I investigated how to minimize the estimation bias for the scattering mechanisms so that the derived SHP's reduces speckle while preserving spatial detail in a PolSAR intensity image.

I applied the Cloude-Pottier eigenvalue-eigenvector decomposition to determine the scattering parameters $(\alpha, \mathrm{H})$ to minimize the scattering mechanism estimation bias. The application of the Cloude-Pottier decomposition as opposed to model based decomposition such as the Freeman-Durden target decomposition minimized the bias because the Cloude-Pottier decomposition does not require orientation angle 
compensation and a-priori information. I applied the Fuzzy $H / \alpha$ Wishart classification to properly model the class mixture around class boundaries and classify the scattering mechanisms. The application of the Fuzzy $H / \alpha$ Wishart classification improved the classification accuracy when compared with the crisp Wishart $H / \alpha$ classification. I also applied a coarse and fine pixel selection that groups the pixels into their canonical scattering mechanisms for spatial filtering. The application of the coarse and fine pixel selection helped retain the spatial details in the PolSAR image. I iteratively refined the estimate of the coherency matrix by gradually increasing the estimation window which significantly improved the scattering mechanism and coherence matrix estimate which subsequently improved the spatial filters accuracy. I evaluated the efficiency of the proposed method by comparing the result from the proposed method with other state of the art PolSAR speckle filters both on simulated and real data.

It is concluded that using dominant scattering mechanism within pixels in addition to the intensity statistics is an effective way to estimate the coherency matrix in PolSAR data. The strength of the developed statistically homogeneous pixels selecting method lies in iteratively improving the estimates of the coherency matrix and scattering mechanism classification results.

The following research question has been answered:

- How can polarimetric information be used to devise spatially adaptive filter that is suitable for properly estimating coherency matrix?

The most powerful aspect of polarimetric SAR is its ability to identify different target scattering mechanisms within a pixel. This unique capability has been exploited for terrain classification. Similarly the target scattering mechanism was used to derive statistically homogeneous pixels in the estimation of coherency matrix. The challenge was minimizing the bias in the target decomposition and classification procedures. I overcame this challenge by initializing the decomposition and classification from a small boxcar filter and iteratively increasing the samples used in the decomposition until a quality threshold is reached. This was shown to compare favorably with other statistically homogeneous pixel selection methods that were based on only intensity statistics.

- Objective 3: To improve the coherence estimate of DS candidates by removing incoherent scatterers.

In this objective, I investigated how to improve the interferometric phase quality of distributed scatterers by selecting coherent scatterers in the pixel. I initially applied scattering property based adaptive filtering to preserve the PS candidates and improve the signal to noise 
ratio of the DS candidates. Moreover, I derived a differential phase entropy threshold to identify the target DS candidates for adaptive removal of incoherent scattering mechanisms. I applied the eigenvalue decomposition on the constructed interferometric coherence matrix to reconstruct the interferometric coherency matrix for the dominant scatterers. In addition, I analyzed how the secondary scattering mechanisms within the pixel interfere with the dominant scattering mechanism. I selected the scatterers that are in-phase with the dominant scattering mechanism and removed the scatterers that interfere destructively. In this way I removed the incoherent scatterers which improved the interferometric coherence of the resolution cell. I then applied an average coherence threshold to select the PS and DS candidates to process in a multi-temporal InSAR method to derive ground deformation. I concluded that the proposed method significantly improves interferometric coherence in distributed scatterers. Moreover, utilizing scattering properties in addition to pixel intensity statistics improved the accuracy of selection pixels to establishing local homogeneity for the application of spatially adaptive filtering. Furthermore, the adaptive selection of secondary scatterers that are coherent improved the pixel coherence estimate. Consequently, this increased the number of measurement pixels available for for deformation measurement. The major draw back in this technique is its computational intensity because it relies on exhausting search optimization to select scattering mechanism that interfere constructively with the dominant scattering mechanism. This is particularly severe when processing large image scenes with millions of pixels.

It is concluded that the proposed method significantly improves interferometric coherence estimate in distributed scatterers at the cost of computational time.

The following research question has been answered:

- Which polarimetric parameter can be used to differentiate permanent scatterers from distributed scatterers.

Phase entropy was important in identifying whether there was a single or multiple dominant scatterers within the resolution cell thereby effectively differentiating PS and DS candidates.

- How can the noisy scattering mechanisms within a resolution cell be eliminated to improve the interferometric coherence estimate.

The noisy scattering mechanisms was distinguished by implementing an exhaustive search optimization to select coherent secondary scattering mechanisms. Elimination of the noisy scattering mechanisms was accomplished by selecting the scattering mechanisms that are in-phase with the dominant scattering mechanisms. This 
improved the coherence estimate. Consequently, this increased the number of measurement pixels available for deformation measurement. Since the main drawback from this technique is the computational time, by applying a threshold on the eigenvalues of scattering mechanisms we can limit the number of secondary scattering mechanism that can be considered, thereby significantly improving the computational time.

\subsection{Reflections}

This thesis research focused on improving the phase quality of distributed scatterers (DS) in differential SAR interferometry context by exploiting the polarimetric diversity provided from fully and dual polarized SAR images. This was partly accomplished in three steps:

1. Polarimetric optimization over distributed scatterers and coherence phase center decomposition to evaluate and quantify the coherence estimation bias, improvement in number of measurement pixels and the improvement in measurement accuracy.

2. Exploiting polarimetric scattering properties to more accurately estimate coherency matrix from distributed scatterers in polarimetric SAR and polarimetric interferometric SAR images and

3. Applying the scattering property based statistically homogeneous pixels selection method, introducing an entropy of interferometric phases to select distributed scatterer candidates and selecting only the secondary scattering mechanisms that are in-phase with the dominant scattering mechanisms effectively removing noisy scattering mechanisms.

These steps contributed to the improvement of quality in DInSAR analysis in a natural environment. Since deformation resulting in landslides, volcanic eruptions and earthquakes may originate in a natural environment, the high accuracy measurement of these displacements helps to understand the mechanism and progression of these natural hazards.

The application of polarimetric optimization on distributed scatterers improved the estimation of coherence of the pixel since polarimetric optimization routines look for the polarization state that maximized the interferometric coherence. Besides the validation against GPS measurements, the main contribution is a coherence decomposition study that assessed the quality of the optimized coherences in terms of ground deformation, instead of the numerical value of coherence by itself. This aspect is especially relevant in vegetated areas, as discussed in Chapter 3 since the phase centers of different polarimetric channels (e.g. the optimum ones) can move away from the ground surface, hence not being really good from the final application 
viewpoint. Even though the coherence decomposition provides possible deformation measurements on vegetated areas, the interferometric phase is severely affected by temporal decorrelation especially for data acquired from spaceborne platforms with longer temporal baseline. Whenever there is a relatively fast displacement within a short time, temporal decorrelation may be smaller so a reliable deformation can be retrieved over densely vegetated regions. This would further improve the usability of the deformation measurements.

Estimation of the SAR covariance matrix has been an active research topic to preserve the information of interest from SAR backscattered signal. In this research, I attempted to use both the intensity and the scattering mechanism that can be derived from fully or partially polarized SAR images to select SHP's. This approach uses the pixel backscatter intensity in addition to pixel scattering mechanisms as a homogeneity measure between pixels. Hence its performance is superior to other SHP selection methods that are based only on pixel intensity statistics. In addition, the method is devised for a single PolSAR image coherency matrix estimation but it can easily be adapted to multi-temporal Polarimetric SAR data stack be it either dual polarized or fully polarized. A major challenge for doing this is the computational complexity. For small area image scenes the computational time may be small but for recently launched partially polarized ScanSAR images such as Sentinel-1 data sets consisting of millions of pixels the computational burden may be too high. Since the efficiency of the scattering property based speckle filter is based on accurately applying target decomposition theorems and classifying the scattering mechanisms, future work should investigate a target decomposition theorem with minimum computational burden and maximum accuracy.

In addition to applying spatially adaptive filtering to improve the signal to noise ratio, additional processing of DS candidates to remove noise is required. In this regard, two unique accomplishments were achieved. The first is the selection of of ideal DS candidates using phase entropy. The second is removing incoherent scattering mechanisms within a DS pixel. The approach proposed in the literature relied on identifying DS candidates by using a threshold of clustered pixels determined by statistical measures. DS candidates however should be identified on whether a single dominant scatterer exists within the resolution cell or there are multiple scatterers within the resolution cell. In this way, utilizing phase entropy measure was effective in distinguishing the DS candidates for further exploitation. The interference pattern of the secondary scatterers with the dominant scatterer was important in significantly improving the signal to noise ratio of the resolution cell thus improving the coherence. Selection of the coherent scattering mechanisms that maximizes the average coherence of the resolution cell requires an exhaustive search technique. This puts additional computational burden to an already computationally intensive PSI analysis. Hence, for large scenes consisting of millions of pixels, application of this technique is impractical. Hence, minimization of the computational burden should be investigated. 


\subsection{Recommendations}

This thesis investigated how to improve the differential phase quality of distributed scatterers from fully and partially polarized SAR images for an improved ground displacement measurement. The proposed methods improved the phase quality but had several limitations. The following recommendations are made:

1. Temporal decorrelation remains the main problem affecting repeat pass SAR interferometry. Hence, the fusion of multi-sensor fully polarimetric SAR data should be investigated to minimize the temporal baseline between subsequent image acquisitions.

2. Computational efficiency of the proposed methodologies is a significant drawback especially in the processing of large image scenes. A computationally efficient target decomposition method should be adopted as a trade off with estimation bias especially in small scale deformation mapping applications. In addition, it was shown in the thesis dual polarization SAR data can provide significant improvement when compared with the traditional single polarization SAR data. Hence, coherence optimization and target decomposition method pertaining to dual polarized SAR images should be further explored.

3. The proposed methods rely on the polarimetric coherency and covariance matrices. Unfortunately knowledge about the uncertainty that arises from the estimation of these matrices is lacking in the literature so it should be addressed in future studies.

4. Regretfully due to lack of in-situ measurements, the ground deformation results obtained from adaptively selecting the constructively interfering scattering mechanisms to increase the pixel coherence could not be validated and quantified with respect to in-situ measurements. This should be addressed in future work.

5. Following the previous recommendation, the detection of multiple stable scatterers within the resolution cell should be explored to increase measurement point density in the image and the robustness of the implemented PSI algorithm. 


\section{Bibliography}

Alipour, S., Tiampo, K. F., Samsonov, S. V., González, P. J., 2015. Shortterm surface deformation on the northern hayward fault, CA, and nearby landslides using polarimetric SAR interferometry (PolInSAR). Pure and Applied Geophysics 172 (8), 2179-2193.

Altamimi, Z., Collilieux, X., Métivier, L., 2011. ITRF2008: an improved solution of the international terrestrial reference frame. Journal of Geodesy 85 (8), 457-473.

Amelung, F., Galloway, D. L., Bell, J. W., Zebker, H. A., Laczniak, R. J., 1999. Sensing the ups and downs of las vegas: InSAR reveals structural control of land subsidence and aquifer-system deformation. Geology 27 (6), $483-486$.

Amelung, F., Jónsson, S., Zebker, H., Segall, P., 2000. Widespread uplift and trapdoor faulting on Galápagos volcanoes observed with radar interferometry. Nature 407 (6807), 993.

Belachew, M., Ebinger, C., Coté, D., Keir, D., Rowland, J., Hammond, J., Ayele, A., 2011. Comparison of dike intrusions in an incipient seafloorspreading segment in Afar, Ethiopia: Seismicity perspectives. Journal of Geophysical Research: Solid Earth 116 (B06405), 1-23.

Berardino, P., Fornaro, G., Lanari, R., Sansosti, E., 2002. A new algorithm for surface deformation monitoring based on small baseline differential SAR interferograms. IEEE Transactions on Geoscience and Remote Sensing 40 (11), 2375-2383.

Bert, M. K., 2006. Radar interferometry: Persistent scatterers technique. The Netherlands: Springer.

Boerner, W.-M., 2006. Recent advances in polarimetry and polarimetric interferometry. In: IEEE International Geoscience and Remote Sensing Symposium, IGARSS. IEEE, pp. 49-51.

Boykov, Y., Veksler, O., Zabih, R., 2001. Fast approximate energy minimization via graph cuts. IEEE Transactions on Pattern Analysis and Machine Intelligence 23 (11), 1222-1239.

Buckreuss, S., Balzer, W., Mühlbauer, P., Werninghaus, R., Pitz, W., 2003. The Terrasar-x satellite project. In: IEEE International Geoscience and remote sensing symposium, IGARSS. Vol. 5. IEEE, pp. 3096-3098. 
Cao, N., Lee, H., Jung, H. C., 2016. A phase-decomposition-based PSInSAR processing method. IEEE Transactions on Geoscience and Remote Sensing 54 (2), 1074-1090.

Cardoso, J.-F., Souloumiac, A., 1996. Jacobi angles for simultaneous diagonalization. SIAM journal on matrix analysis and applications 17 (1), $161-164$.

Chen, C. W., Zebker, H. A., 2002. Phase unwrapping for large SAR interferograms: Statistical segmentation and generalized network models. IEEE Transactions on Geoscience and Remote Sensing 40 (8), 1709-1719.

Chen, J., Chen, Y., An, W., Cui, Y., Yang, J., 2011. Nonlocal filtering for polarimetric SAR data: A pretest approach. IEEE Transactions on Geoscience and Remote Sensing 49 (5), 1744-1754.

Cloude, S., 2009. Polarisation: applications in remote sensing. Oxford University Press.

Cloude, S., Papathanassiou, K., 2003. Three-stage inversion process for polarimetric SAR interferometry. IEEE Proceedings on Radar, Sonar and Navigation 150 (3), 125-134.

Cloude, S. R., 1990. Conditions for the physical realisability of matrix operators in polarimetry. In: 33rd Annual Techincal Symposium. International Society for Optics and Photonics, pp. 177-187.

Cloude, S. R., Papathanassiou, K. P., 1998. Polarimetric SAR interferometry. IEEE Transactions on Geoscience and Remote Sensing 36 (5), 1551-1565.

Cloude, S. R., Pottier, E., 1995. Concept of polarization entropy in optical scattering. Optical Engineering 34 (6), 1599-1610.

Cloude, S. R., Pottier, E., 1996. A review of target decomposition theorems in RADAR polarimetry. IEEE Transactions on Geoscience and Remote Sensing 34 (2), 498-518.

Cloude, S. R., Pottier, E., 1997. An entropy based classification scheme for land applications of polarimetric SAR. IEEE Transactions on Geoscience and Remote Sensing 35 (1), 68-78.

Colesanti, C., Ferretti, A., Novali, F., Prati, C., Rocca, F., 2003. Sar monitoring of progressive and seasonal ground deformation using the permanent scatterers technique. IEEE Transactions on Geoscience and Remote Sensing 41 (7), 1685-1701.

Colesanti, C., Wasowski, J., 2006. Investigating landslides with space-borne synthetic aperture radar (SAR) interferometry. Engineering geology 88 (3), 173-199.

Colin, E., Titin-Schnaider, C., Tabbara, W., 2006. An interferometric coherence optimization method in RADAR polarimetry for high-resolution imagery. IEEE Transactions on Geoscience and Remote Sensing 44 (1), $167-175$.

Crosetto, M., Biescas, E., Duro, J., Closa, J., Arnaud, A., 2008. Generation of advanced ERS and envisat interferometric SAR products using the stable point network technique. Photogrammetric Engineering \& Remote Sensing 74 (4), 443-450. 
Crosetto, M., Monserrat, O., Cuevas-González, M., Devanthéry, N., Crippa, B., 2016. Persistent scatterer interferometry: a review. ISPRS Journal of Photogrammetry and Remote Sensing 115, 78-89.

Crosetto, M., Monserrat, O., Iglesias, R., Crippa, B., 2010. Persistent scatterer interferometry. Photogrammetric Engineering \& Remote Sensing 76 (9), 1061-1069.

Cumming, I. G., Wong, F. H., 2005. Digital processing of synthetic aperture radar data. Artech house 1 (2), 3.

Deledalle, C.-A., Denis, L., Tupin, F., Reigber, A., Jager, M., 2015. NL-SAR: A unified nonlocal framework for resolution-preserving (Pol)(In) SAR denoising. IEEE Transactions on Geoscience and Remote Sensing 53 (4), 2021-2038.

Elachi, C., Van Zyl, J. J., 2006. Introduction to the physics and techniques of remote sensing. Vol. 28. John Wiley \& Sons.

ESA, 2016. Tracking every move. Accessed: 2017-11-21. URL http://www.esa.int/spaceinimages/Images/2016/06/ Tracking_every_move

Ferretti, A., Fumagalli, A., Novali, F., Prati, C., Rocca, F., Rucci, A., 2011. A new algorithm for processing interferometric data-stacks: SqueeSAR. IEEE Transactions on Geoscience and Remote Sensing 49 (9), 3460-3470.

Ferretti, A., Prati, C., Rocca, F., 2001. Permanent scatterers in SAR interferometry. IEEE Transactions on Geoscience and Remote Sensing 39 (1), 8-20.

Ferro-Famil, L., Neumann, M., Huang, Y., 2009. Multi-baseline POL-InSAR statistical techniques for the characterization of distributed media. In: IEEE International Geoscience and Remote Sensing Symposium, IGARSS. Vol. 3. pp. 971-974.

Ferro-Famil, L., Pottier, E., Lee, J., 2002. Classification and interpretation of polarimetric interferometric SAR data. In: IEEE International Geoscience and Remote Sensing Symposium, IGRASS. Vol. 1. IEEE, pp. 635-637.

Fletcher, R., Reeves, C. M., 1964. Function minimization by conjugate gradients. The computer journal 7 (2), 149-154.

Fornaro, G., Verde, S., Reale, D., Pauciullo, A., 2015. CAESAR: An approach based on covariance matrix decomposition to improve multibaselinemultitemporal interferometric SAR processing. IEEE Transactions on Geoscience and Remote Sensing 53 (4), 2050-2065.

Foucher, S., Lopez-Martinez, C., 2014. Analysis, evaluation, and comparison of polarimetric SAR speckle filtering techniques. IEEE Transactions on Image Processing 23 (4), 1751-1764.

Freeman, A., Durden, S. L., 1998. A three-component scattering model for polarimetric SAR data. IEEE Transactions on Geoscience and Remote Sensing 36 (3), 963-973.

Goel, K., Adam, N., 2014. A distributed scatterer interferometry approach for precision monitoring of known surface deformation phenomena. IEEE Transactions on Geoscience and Remote Sensing 52 (9), 5454-5468. 
Goldstein, R., Zebker, H., Werner, C., 1988. Satellite RADAR interferometrytwo-dimensional phase unwrapping. Radio science 23 (4), 713-720.

Goodman, J. W., 1976. Some fundamental properties of speckle. JOSA 66 (11), 1145-1150.

Goodman, N., 1963. Statistical analysis based on a certain multivariate complex Gaussian distribution (an introduction). The Annals of Mathematical Statistics 34 (1), 152-177.

Hammond, J., Kendall, J.-M., Stuart, G., Keir, D., Ebinger, C., Ayele, A., Belachew, M., 2011. The nature of the crust beneath the afar triple junction: Evidence from receiver functions. Geochemistry, Geophysics, Geosystems 12 (12), 1-24.

Hanssen, R. F., 2001. RADAR interferometry: data interpretation and error analysis. Vol. 2. Springer Science \& Business Media.

Hooper, A., 2008. A multi-temporal InSAR method incorporating both persistent scatterer and small baseline approaches. Geophysical Research Letters 35 (16).

Hooper, A., Segall, P., Zebker, H., 2007. Persistent scatterer interferometric synthetic aperture radar for crustal deformation analysis, with application to Volcán Alcedo, Galápagos. Journal of Geophysical Research: Solid Earth 112 (B7), 1978-2012.

Hooper, A., Zebker, H., Segall, P., Kampes, B., 2004. A new method for measuring deformation on volcanoes and other natural terrains using insar persistent scatterers. Geophysical research letters 31 (23).

Iglesias, R., Monells, D., Fabregas, X., Mallorqui, J. J., Aguasca, A., LopezMartinez, C., 2014. Phase quality optimization in polarimetric differential SAR interferometry. IEEE transactions on geoscience and remote sensing $52(5), 2875-2888$.

Iglesias, R., Monells, D., Lopez-Martinez, C., Mallorqui, J. J., Fabregas, X., Aguasca, A., 2015. Polarimetric optimization of temporal sublook coherence for DInSAR applications. IEEE Geoscience and Remote Sensing Letters 12 (1), 87-91.

Jagdhuber, T., Stockamp, J., Hajnsek, I., Ludwig, R., 2014. Identification of soil freezing and thawing states using SAR polarimetry at C-band. Remote Sensing 6 (3), 2008-2023.

Ji, K., Wu, Y., 2015. Scattering mechanism extraction by a modified cloudepottier decomposition for dual polarization SAR. Remote Sensing 7 (6), $7447-7470$.

Jiang, M., Ding, X., Hanssen, R. F., Malhotra, R., Chang, L., 2015. Fast statistically homogeneous pixel selection for covariance matrix estimation for multitemporal insar. IEEE Transactions on Geoscience and Remote Sensing 53 (3), 1213-1224.

Kampes, B. M., Hanssen, R. F., 2004. Ambiguity resolution for permanent scatterer interferometry. IEEE Transactions on Geoscience and Remote Sensing 42 (11), 2446-2453. 
Keir, D., Pagli, C., Bastow, I. D., Ayele, A., 2011. The magma-assisted removal of Arabia in Afar: Evidence from dike injection in the Ethiopian rift captured using InSAR and seismicity. Tectonics 30 (2), 1-13.

Kelly, W. E., Painter, J. H., 1996. Hypertrapezoidal fuzzy membership functions. In: Proceedings of the Fifth IEEE International Conference on Fuzzy Systems, 1996. Vol. 2. IEEE, pp. 1279-1284.

Kersten, P. R., Lee, J.-S., Ainsworth, T. L., 2005. Unsupervised classification of polarimetric synthetic aperture radar images using fuzzy clustering and EM clustering. IEEE Transactions on Geoscience and Remote Sensing $43(3), 519-527$.

King, R., Bock, Y., 2005. Documentation for the GAMIT GPS processing software release 10.2. Massachusetts Institute of Technology, Cambridge, MA.

Krieger, G., Papathanassiou, K. P., Cloude, S. R., 2005. Spaceborne polarimetric SAR interferometry: Performance analysis and mission concepts. EURASIP Journal on Applied Signal Processing 2005, 3272-3292.

Lee, J.-S., 1980. Digital image enhancement and noise filtering by use of local statistics. IEEE Transactions on Pattern Analysis and Machine Intelligence (2), 165-168.

Lee, J.-S., 1981. Speckle analysis and smoothing of synthetic aperture radar images. Computer Graphics and Image Processing 17 (1), 24-32.

Lee, J.-S., Ainsworth, T. L., Kelly, J. P., Lopez-Martinez, C., 2008. Evaluation and bias removal of multilook effect on entropy/alpha/anisotropy in polarimetric SAR decomposition. IEEE Transactions on Geoscience and Remote Sensing 46 (10), 3039-3052.

Lee, J.-S., Cloude, S. R., Papathanassiou, K. P., Grunes, M. R., Woodhouse, I. H., 2003. Speckle filtering and coherence estimation of polarimetric SAR interferometry data for forest applications. IEEE Transactions on Geoscience and Remote Sensing 41 (10), 2254-2263.

Lee, J.-S., Grunes, M. R., Ainsworth, T. L., Du, L.-J., Schuler, D. L., Cloude, S. R., 1999a. Unsupervised classification using polarimetric decomposition and the complex Wishart classifier. IEEE Transactions on Geoscience and Remote Sensing 37 (5), 2249-2258.

Lee, J.-S., Grunes, M. R., De Grandi, G., 1999b. Polarimetric SAR speckle filtering and its implication for classification. IEEE Transactions on Geoscience and Remote Sensing 37 (5), 2363-2373.

Lee, J.-S., Grunes, M. R., Kwok, R., 1994a. Classification of multi-look polarimetric SAR imagery based on complex wishart distribution. International Journal of Remote Sensing 15 (11), 2299-2311.

Lee, J.-S., Grunes, M. R., Pottier, E., 2001. Quantitative comparison of classification capability: Fully polarimetric versus dual and single-polarization SAR. IEEE Transactions on Geoscience and Remote Sensing 39 (11), 2343-2351. 
Lee, J.-S., Grunes, M. R., Schuler, D. L., Pottier, E., Ferro-Famil, L., 2006. Scattering-model-based speckle filtering of polarimetric SAR data. IEEE Transactions on Geoscience and Remote Sensing 44 (1), 176-187.

Lee, J.-S., Hoppel, K. W., Mango, S. A., Miller, A. R., 1994b. Intensity and phase statistics of multilook polarimetric and interferometric SAR imagery. IEEE Transactions on Geoscience and Remote Sensing 32 (5), 1017-1028.

Lee, J.-S., Pottier, E., 2009. Polarimetric RADAR imaging: from basics to applications. CRC press.

Lee, J.-S., Wen, J.-H., Ainsworth, T. L., Chen, K.-S., Chen, A. J., 2009. Improved sigma filter for speckle filtering of SAR imagery. IEEE Transactions on Geoscience and Remote Sensing 47 (1), 202-213.

Lin, K.-F., Perissin, D., 2017. Identification of statistically homogeneous pixels based on one-sample test. Remote Sensing 9 (1), 37.

Lopez-Martinez, C., Fabregas, X., 2008. Model-based polarimetric SAR speckle filter. IEEE Transactions on Geoscience and Remote Sensing 46 (11), 3894-3907.

Lopez-Martinez, C., Pottier, E., Cloude, S. R., 2005. Statistical assessment of eigenvector-based target decomposition theorems in radar polarimetry. IEEE Transactions on Geoscience and Remote Sensing 43 (9), 2058-2074.

Lopez-Sanchez, J. M., Hajnsek, I., Ballester-Berman, J. D., 2012. First demonstration of agriculture height retrieval with PolInSAR airborne data. IEEE Geoscience and Remote Sensing Letters 9 (2), 242-246.

Massonnet, D., Rossi, M., Carmona, C., Adragna, F., Peltzer, G., Feigl, K., Rabaute, T., 1993. The displacement field of the landers earthquake mapped by radar interferometry. Nature 364 (6433), 138-142.

Mora, O., Mallorqui, J. J., Broquetas, A., 2003. Linear and nonlinear terrain deformation maps from a reduced set of interferometric SAR images. IEEE Transactions on Geoscience and Remote Sensing 41 (10), 2243-2253.

Morena, L., James, K., Beck, J., 2004. An introduction to the RADARSAT-2 mission. Canadian Journal of Remote Sensing 30 (3), 221-234.

Mullissa, A. G., Tolpekin, V., Stein, A., 2017a. Scattering property based contextual PolSAR speckle filter. International Journal of Applied Earth Observation and Geoinformation 63, 78-89.

Mullissa, A. G., Tolpekin, V., Stein, A., Perissin, D., 2017b. Polarimetric differential SAR interferometry in an arid natural environment. International Journal of Applied Earth Observation and Geoinformation 59, 9-18.

Navarro-Sanchez, V. D., Lopez-Sanchez, J. M., 2012. Improvement of persistent-scatterer interferometry performance by means of a polarimetric optimization. IEEE Geoscience and Remote Sensing Letters 9 (4), 609-613.

Navarro-Sanchez, V. D., Lopez-Sanchez, J. M., 2014. Spatial adaptive speckle filtering driven by temporal polarimetric statistics and its application to PSI. IEEE Transactions on Geoscience and Remote Sensing 52 (8), 4548-4557. 
Navarro-Sanchez, V. D., Lopez-Sanchez, J. M., Ferro-Famil, L., 2014. Polarimetric approaches for persistent scatterers interferometry. IEEE Transactions on Geoscience and Remote Sensing 52 (3), 1667-1676.

Navarro-Sanchez, V. D., Lopez-Sanchez, J. M., Vicente-Guijalba, F., 2010. A contribution of polarimetry to satellite differential SAR interferometry: Increasing the number of pixel candidates. IEEE Geoscience and Remote Sensing Letters 7 (2), 276-280.

Neumann, M., Ferro-Famil, L., Reigber, A., 2008. Multibaseline polarimetric SAR interferometry coherence optimization. IEEE Geoscience and Remote Sensing Letters 5 (1), 93-97.

Neumann, M., Ferro-Famil, L., Reigber, A., 2010. Estimation of forest structure, ground, and canopy layer characteristics from multibaseline polarimetric interferometric SAR data. IEEE Transactions on Geoscience and Remote Sensing 48 (3), 1086-1104.

Papathanassiou, K. P., Cloude, S. R., 2001. Single-baseline polarimetric SAR interferometry. IEEE Transactions on Geoscience and Remote Sensing 39 (11), 2352-2363.

Park, S.-E., Moon, W. M., 2007. Unsupervised classification of scattering mechanisms in polarimetric SAR data using fuzzy logic in entropy and alpha plane. IEEE Transactions on Geoscience and Remote Sensing 45 (8), 2652-2664.

Perissin, D., Wang, T., 2012. Repeat-pass SAR interferometry with partially coherent targets. IEEE Transactions on Geoscience and Remote Sensing 50 (1), 271-280.

Perissin, D., Wang, Z., Wang, T., 2011. The SARPROZ InSAR tool for urban subsidence/manmade structure stability monitoring in China. Proceedings of the ISRSE, Sidney, Australia 1015.

Pipia, L., Fabregas, X., Aguasca, A., Lopez-Martinez, C., Duque, S., Mallorqui, J. J., Marturia, J., 2009. Polarimetric differential SAR interferometry: First results with ground-based measurements. IEEE Geoscience and Remote Sensing Letters 6 (1), 167-171.

Pottier, E., Ferro-Famil, L., Allain, S., Cloude, S., Hajnsek, I., Papathanassiou, K., Moreira, A., Williams, M., Minchella, A., Lavalle, M., et al., 2009. Overview of the polsarpro v4. 0 software. the open source toolbox for polarimetric and interferometric polarimetric SAR data processing. In: IEEE International Geoscience and Remote Sensing Symposium, IGRASS. Vol. 4. IEEE, pp. IV-936.

Rosenqvist, A., Shimada, M., Ito, N., Watanabe, M., 2007. ALOS PALSAR: A pathfinder mission for global-scale monitoring of the environment. IEEE Transactions on Geoscience and Remote Sensing 45 (11), 3307-3316.

Roth, S., Black, M. J., 2005. Fields of experts: A framework for learning image priors. In: IEEE Computer Society Conference on Computer Vision and Pattern Recognition, 2005. CVPR 2005. Vol. 2. IEEE, pp. 860-867. 
Sagues, L., Lopez-Sanchez, J. M., Fortuny, J., Fabregas, X., Broquetas, A., Sieber, A. J., 2000. Indoor experiments on polarimetric SAR interferometry. IEEE Transactions on Geoscience and Remote Sensing 38 (2), 671-684.

Samiei-Esfahany, S., Martins, J. E., van Leijen, F., Hanssen, R. F., 2016. Phase estimation for distributed scatterers in InSAR stacks using integer least squares estimation. IEEE Transactions on Geoscience and Remote Sensing 54 (10), 5671-5687.

Samsonov, S., Tiampo, K., 2011. Polarization phase difference analysis for selection of persistent scatterers in SAR interferometry. IEEE Geoscience and Remote Sensing Letters 8 (2), 331-335.

Stone, M. H., 1932. Linear transformations in Hilbert space and their applications to analysis. Vol. 15. American Mathematical Society.

Stratton, J., 1941. Electromagnetic theory, Mcgrow-Hill, New-York.

Tabb, M., Orrey, J., Flynn, T., Carande, R., 2002. Phase diversity: a decomposition for vegetation parameter estimation using polarimetric SAR interferometry. In: Proceedings of EUSAR 2002. Vol. 2. pp. 721-724.

Touzi, R., Lopes, A., Bruniquel, J., Vachon, P. W., 1999. Coherence estimation for SAR imagery. IEEE Transactions on Geoscience and Remote Sensing 37 (1), 135-149.

Vasile, G., Trouvé, E., Lee, J.-S., Buzuloiu, V., 2006. Intensity-driven adaptive-neighborhood technique for polarimetric and interferometric SAR parameters estimation. IEEE Transactions on Geoscience and Remote Sensing 44 (6), 1609-1621.

Wang, T., Liao, M., Perissin, D., 2010. InSAR coherence-decomposition analysis. IEEE Geoscience and Remote Sensing Letters 7 (1), 156-160.

Wang, Y., Davis, F., 1997. Decomposition of polarimetric synthetic aperture radar backscatter from upland and flooded forests. International Journal of Remote Sensing 18 (6), 1319-1332.

Woodhouse, I. H., 2005. Introduction to microwave remote sensing. CRC press.

Wu, B., Tong, L., Chen, Y., He, L., 2015. New methods in multibaseline polarimetric SAR interferometry coherence optimization. IEEE Geoscience and Remote Sensing Letters 12 (10), 2016-2020.

Xiang, Z., Wang, K., Liu, X., 2009. A model-spectrum-based flattening algorithm for airborne single-pass SAR interferometry. IEEE Geoscience and Remote Sensing Letters 6 (2), 307-311.

Yajima, Y., Yamaguchi, Y., Sato, R., Yamada, H., Boerner, W.-M., 2008. PolSAR image analysis of wetlands using a modified four-component scattering power decomposition. IEEE Transactions on Geoscience and Remote Sensing 46 (6), 1667-1673.

Yamaguchi, Y., Sato, A., Boerner, W.-M., Sato, R., Yamada, H., 2011. Fourcomponent scattering power decomposition with rotation of coherency matrix. IEEE Transactions on Geoscience and Remote Sensing 49 (6), 2251-2258. 
Zadeh, L. A., 1965. Fuzzy sets. Information and control 8 (3), 338-353.

Zebker, H., Villasenor, J., et al., 1992. Decorrelation in interferometric RADAR echoes. IEEE Transactions on Geoscience and Remote Sensing 30 (5), 950-959.

Zebker, H. A., Rosen, P. A., Goldstein, R. M., Gabriel, A., Werner, C. L., 1994. On the derivation of coseismic displacement fields using differential radar interferometry: The landers earthquake. Journal of Geophysical Research: Solid Earth 99 (B10), 19617-19634.

Zhong, H., Zhang, J., Liu, G., 2014. Robust polarimetric SAR despeckling based on nonlocal means and distributed lee filter. IEEE Transactions on Geoscience and Remote Sensing 52 (7), 4198-4210. 



\section{Biography}

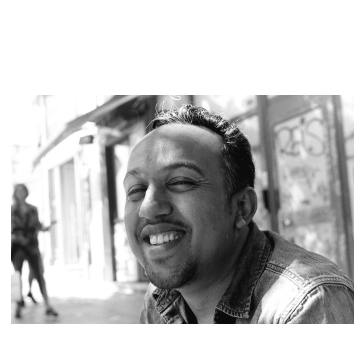

Adugna Mullissa obtained his BSc degree in Geology and geophysics from Addis Abeba University, Ethiopia in 2004, and the MSc degree in Earth sciences with focus on Remote Sensing for Earth science applications at the Addis Abeba University in 2008, Addis Abeba, Ethiopia. In November 2013, he joined the Faculty of Geo-Information Science and Earth Observation (ITC) at the University of Twente for his $\mathrm{PhD}$ research which culminated in this PhD thesis. From September 2016 to January 2017, he was a visiting research scholar at Lyle school of civil engineering, Purdue University, United States. His research interests include Polarimetric SAR data denoising, multi-temporal polarimetric differential SAR interferometry and pattern recognition and machine learning for Polarimetric SAR data. 



\section{Author's publications}

\section{ISI journal article under review}

- Mullissa, A.G., Perissin, D., Tolpekin, V., Stein, A. Polarimetric scattering property based distributed scatterer processing method for PSI applications. IEEE Transactions in Geoscience and Remote Sensing (Minor revision).

\section{Published ISI journal articles}

- Mullissa, A.G., Tolpekin, V., Stein, A., Perissin, D., 2017. Polarimetric differential SAR interferometry in an arid natural environment. International Journal of Applied Earth Observation and Geoinformation 59:9-18, 2017.

- Mullissa, A.G., Tolpekin, V., Stein, A., 2017. Scattering property based contextual PolSAR speckle filter. International Journal of Applied Earth Observation and Geoinformation 63:78-89, 2017.

\section{Conference presentation}

- Mullissa, A.G., Perissin, D., Tolpekin, V., Stein, A., 2017. Scattering property based adaptive filtering of dual polarization Sentinel-1 data for PSI application. Fringe 2017, Helsinki, Finland. 



\section{ITC Dissertation List}

http://www.itc.nl/Pub/research_programme/Research-review-and-output/PhDGraduates 